\title{
Organ-preservation in rectal cancer : can new MRI- techniques improve patient selection?
}

Citation for published version (APA):

Martens, M. H. (2015). Organ-preservation in rectal cancer : can new MRl-techniques improve patient selection? [Doctoral Thesis, Maastricht University]. Uitgeverij BOXPress.

https://doi.org/10.26481/dis.20151204mm

Document status and date:

Published: 01/01/2015

DOI:

10.26481/dis.20151204mm

Document Version:

Publisher's PDF, also known as Version of record

\section{Please check the document version of this publication:}

- A submitted manuscript is the version of the article upon submission and before peer-review. There can be important differences between the submitted version and the official published version of record.

People interested in the research are advised to contact the author for the final version of the publication, or visit the DOI to the publisher's website.

- The final author version and the galley proof are versions of the publication after peer review.

- The final published version features the final layout of the paper including the volume, issue and page numbers.

Link to publication

\footnotetext{
General rights rights.

- You may freely distribute the URL identifying the publication in the public portal. please follow below link for the End User Agreement:

www.umlib.nl/taverne-license

Take down policy

If you believe that this document breaches copyright please contact us at:

repository@maastrichtuniversity.nl

providing details and we will investigate your claim.
}

Copyright and moral rights for the publications made accessible in the public portal are retained by the authors and/or other copyright owners and it is a condition of accessing publications that users recognise and abide by the legal requirements associated with these

- Users may download and print one copy of any publication from the public portal for the purpose of private study or research.

- You may not further distribute the material or use it for any profit-making activity or commercial gain

If the publication is distributed under the terms of Article $25 \mathrm{fa}$ of the Dutch Copyright Act, indicated by the "Taverne" license above, 


\section{ORGAN-PRESERVATION IN RECTAL CANCER}

Can new MRI-techniques improve patient selection? 
(c) M.H. Martens, Maastricht 2015

All rights reserved. No part of this publication may be reproduced, stored in a retrieval database, or published in any form or by any means, electronic, mechanical or photocopying, recording, or otherwise, without the prior written permission of the publisher.

ISBN 978-90-9029297-7

Cover Design: $\quad$ proefschriftmaken.nl

Printed by: $\quad$ proefschriftmaken.nl | Uitgeverij BOXPress

Published by: $\quad$ proefschriftmaken.nl| Uitgeverij BOXPress

Printing of this thesis was financially supported by: Philips Medical Imaging 


\title{
ORGAN-PRESERVATION IN RECTAL CANCER
}

Can new MRI-techniques improve patient selection?

PROEFSCHRIFT

\author{
ter verkrijging van de graad van doctor aan \\ de Universiteit Maastricht, \\ op gezag van de Rector Magnificus, \\ Prof dr. L.L.G. Soete \\ volgens het besluit van het College van Decanen, \\ in het openbaar te verdedigen \\ op vrijdag 4 december 2015 om 14.00 uur
}

door

Milou Hélène Martens 


\section{Promotores}

Prof. dr. R.G.H. Beets-Tan

Prof. dr. G.L. Beets

\section{Copromotor}

Dr. D.M.J. Lambregts

\section{Beoordelingscommissie}

Prof. dr. J.M.A. van Engelshoven (voorzitter)

Prof. dr. ir. W.H. Backes

Prof. dr. N. Bouvy

Prof. dr. J. Stoker (Academisch Medisch Centrum, Amsterdam)

Prof. dr. M. Verheij (Nederlands Kanker Instituut - Antoni van Leeuwenhoek, Amsterdam) 


\section{CONTENTS}

CHAPTER 1: General introduction

PART I - Organ preservation

CHAPTER 2: Organ preservation in 100 clinical complete responders

after neoadjuvant treatment for rectal cancer: long-term

oncological and functional outcome

\section{PART II - Response assessment after therapy}

CHAPTER 3: Prospective multicenter validation study of MRI volumetry for response assessment after preoperative chemoradiation in rectal cancer: can the results in literature be reproduced

CHAPTER 4: Magnetization Transfer Ratio - A potential biomarker for the assessment of post-radiation fibrosis in patients with rectal cancer

CHAPTER 5: $\quad$ Magnetization Transfer Imaging to assess tumor response after chemoradiotherapy in rectal cancer

\section{PART III - Response prediction before therapy}

CHAPTER 6: Can perfusion MRI predict response to preoperative treatment in rectal cancer?

CHAPTER 7: Non-invasive MR assessment of macroscopic and microscopic vascular abnormalities in the rectal tumor-surrounding mesorectum

CHAPTER 8: General discussion, future perspectives, and recommendations

CHAPTER 9: Valorisation

Summary

Samenvatting

Dankwoord

Curriculum Vitae 


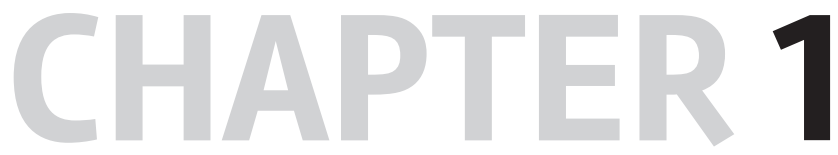

General introduction 
In the Netherlands, approximately 4000 patients are diagnosed with rectal cancer every year ${ }^{1}$. For a long time, the main challenge of rectal cancer was the high rate of local recurrences of up to $40 \%{ }^{2}$. The introduction of standardized surgery, i.e. Total Mesorectal Excision (TME) ${ }^{3}$, and preoperative (chemo)radiotherapy ${ }^{4,5}$, revolutionized rectal cancer management and significantly reduced local recurrence rates to $<10 \%$. In the Dutch TME trial, addition of a preoperative short course of 5x5 Gray of radiotherapy resulted in a 10-year local recurrence rate of $5 \%$ in the irradiated patients compared to $11 \%$ in the patients who were treated with surgery only ${ }^{6}$. Moreover, we learned from the Dutch TME trial that not all patients benefit equally from short course radiotherapy; patients with stage I disease showed low local recurrence rates regardless of the addition of radiotherapy ( $0.5 \mathrm{vs} 0.7 \%, p=0.15)^{5}$. Patients with locally advanced rectal cancer showed a significant decrease in local recurrence with the addition of radiotherapy, but the local recurrence rate remained high (19\% vs 34\%, $p<0.001)^{4}$. In these high risk patients, a short course of radiation will not suffice and better results have been shown for long course neoadjuvant combined chemo- and radiation treatment.

Nowadays, the allocation of neoadjuvant treatment is therefore based on a patient's a priori risk of a local recurrence ${ }^{7}$. The risk stratification is based on assessment of the most important factors for recurrence on MRI. Patients with a low risk are stratified for TME without neoadjuvant treatment, patients with an intermediate risk are treated with a short course of radiotherapy (5x5 Gy) followed by TME, and the high risk patients will undergo a long course of radiotherapy combined with chemotherapy (i.e. chemoradiation or CRT) followed by TME.

\section{Organ preservation}

The primary aim of the CRT is to obtain downsizing or downstaging of the tumor and lymph nodes in order to increase the chance of a complete (RO) resection. In approximately 15$20 \%$ of the patients treated with neoadjuvant CRT followed by surgery, no tumor is found in the resection specimen8. This means that all tumor cells were destroyed by the CRT, which is called a pathological complete response (PCR). Patients with a pCR are known to have a better prognosis than patients in whom residual tumor is still present after CRT and surgery ${ }^{8}$. One could wonder whether major surgery is still justified in these patients with a pCR, considering that TME is associated with a post-operative complication rate of around $28 \%{ }^{9}$, the majority of patients requires a temporary or permanent colostomy, and mortality rates are $4-12 \%{ }^{10}$. These considerations have led to the introduction of the concept of "organpreservation". An alternative for major resection after CRT in good responders with only a small residual tumor (ycT1-2N0) could be a local excision of the tumor remnant by means of a Transanal Endoscopic Microsurgery $(T E M)^{11}$. Another alternative for patients with clinical evidence of a complete response (i.e. no evidence of residual tumor or metastatic lymph nodes) is to omit surgery completely and instead follow patients intensively; the so called 'wait-and-see policy' or 'watch-and-wait strategy'. The watch-and-wait strategy was first described by the group of Habr-Gama et al. ${ }^{12,13}$. They selected clinical complete responders (CCR) with digital rectal examination and endoscopy with promising results ${ }^{14}$. Other groups 
from the USA, Denmark, and Maastricht have performed the watch-and-wait strategy in relatively small series and reported similarly promising results ${ }^{15-18}$.

\section{Issues in organ-preservation}

The main concern of clinicians with organ-preserving treatments is that the available data is insufficient to estimate the oncological safety and there is a potentially increased risk for local recurrence and distant metastasis. Ideally, this evidence should be provided by randomized trials where eligible patients are randomized between organ-preservation or standard TME. However, such trials are very difficult to realize because patients strongly prefer to avoid major surgery and a colostomy. Therefore, large prospective registries with long-term followup are required. Furthermore, it is suggested that patients will benefit from a watch-and-wait policy in terms of sphincter function and quality of life, but so far only one study showed this benefit ${ }^{17}$.

Another major issue is the selection of suitable patients for organ-preservation. If the assessment of response is not accurate and patients with residual tumor or malignant lymph nodes are not identified as such, they will not have radical surgery. These patients will then have an increased risk of a local regrowth and potentially a greater risk for distant metastasis and impaired overall survival. On the other hand, if a clinical complete response or a very good response is not recognized, the options of watch-and-wait or TEM are denied, while these might have been good alternatives with less morbidity and mortality. Several strategies for the selection of eligible patients for organ-preservation have been reported.

Currently, published reports mainly use digital rectal examination (DRE) and endoscopy with biopsies as tools for the response assessment. However, DRE is known to be an unreliable predictor of response ${ }^{19}$. Endoscopic assessment may overestimate response ${ }^{20,21}$ and biopsies are often false-negative ${ }^{22,23}$. So far, only Maas et al. routinely used MR imaging as an additional selection too ${ }^{17}$ but the role of imaging in response assessment and patient selection is not yet consolidated.

\section{Response assessment after therapy}

The value of standard clinical MRI protocols for response assessment is limited with a sensitivity of only $49 \%{ }^{24}$. The main problem is the interpretation of post-radiation fibrosis. On morphological imaging, fibrosis with or without residual tumor remnants look alike, but there is a 50\% chance that an area of apparent fibrosis still contains residual tumor ${ }^{25}$. A quantitative approach of morphologic changes, by means of measuring the tumor length or volume has been suggested to improve the response assessment. However, different methods for measurements and multiple thresholds have been described with variable results in often limited numbers of patients. Therefore, its role has not yet been established. Functional MR imaging techniques are emerging, which do not look at tumor morphology, but target different biological characteristics of the tumor. An intensively investigated technique is diffusion-weighted MRI (DWI) with significantly improved accuracies compared to standard 
MRI, but with limited sensitivity for predicting a complete response ${ }^{26}$. Another functional MR imaging technique that is new on the horizon for cancer imaging is 'Magnetization Transfer' (MT) imaging, which provides information on the presence of macromolecules within a tissue. MT imaging may help us to assess post-radiation fibrosis on a micro-architectural level, since fibrosis is rich of collagens and therefore contains may macromolecules ${ }^{27,28}$.

\section{Response prediction before therapy}

An interesting concept is to shift the focus towards the prediction of response before the onset of therapy. If we could predict early in the treatment process which patients are likely to benefit from neoadjuvant treatment and will undergo a good tumor response, this may offer new perspectives towards more personalized treatment planning. In the near future, this will likely become an increasingly relevant question, since recent and ongoing trials aim to extend the indication for neoadjuvant treatment to patients with low-risk tumors with the aim of achieving a good response and the options of organ-preservation ${ }^{11,29}$. Since these patients would not receive neoadjuvant treatment according to the current guidelines, it is important to have information on the expected response to neoadjuvant treatment to ensure a safe selection of patients that will benefit from such a treatment strategy. Patients with a poor response would still require TME, but suffer the consequences of CRT in terms of morbidity.

Preliminary studies have explored the potential role of functional MR techniques such as DWI and dynamic contrast-enhanced (DCE) MRI for response prediction. The role of DWI is not consolidated yet, since conflicting results have been reported ${ }^{26}$. DCE-MRI provides information on tumor perfusion and vascularization, which is an important factor associated with tumor viability and its sensibility to chemo- and radiation treatment ${ }^{30,31}$. The evidence on DCE-MRI for response prediction to date is limited; some groups reported a high perfusion before onset of therapy in good responders ${ }^{32-37}$, while others found the opposite relation ${ }^{38-40}$. Therefore there is a need for a better understanding of the role of tumor perfusion and its relation to response to therapy.

\section{Aims of this thesis}

- To evaluate the oncological and functional outcome of organ-preserving treatment options after neoadjuvant chemoradiation as an alternative to standard resection

- To explore the role of modern MR imaging techniques to improve patient selection after neoadjuvant therapy for organ-preserving treatments

- To investigate the feasibility of a novel MRI technique to predict before onset of treatment which patients will show a good response to therapy 


\section{Outline of this thesis \\ Part I: Organ preservation}

Chapter 2 discusses the long-term results of a cohort of 100 patients that was treated with organ-preserving treatment options.

\section{Part II: Response assessment after therapy}

Chapter 3 reviews the available literature on tumor size and volume measurements on MRI for response assessment after chemoradiotherapy. Optimal size and volume cut-offs are determined and validated in an independent multicenter cohort.

Chapter 4 explores the potential of the novel MR technique "magnetization transfer" imaging to differentiate between residual tumor and fibrosis after neoadjuvant chemoradiotherapy.

In Chapter 5 the clinical applicability of "magnetization transfer" imaging is evaluated to differentiate between patients with a good and poor response after neoadjuvant therapy.

\section{Part III: Response prediction before therapy}

Chapter 6 focuses on the performance of dynamic contrast enhanced MRI to predict tumor response before the onset of neoadjuvant treatment.

In Chapter 7 the mesorectal vasculature before therapy and its relation to tumor response is investigated.

\section{References}

1. Integraal Kankercentrum Nederland - Cijfers over kanker. 2015. http://www.cijfersoverkanker.nl/ nkr/index.

2. Quirke P, Durdey P, Dixon MF, Williams NS. Local recurrence of rectal adenocarcinoma due to inadequate surgical resection. Histopathological study of lateral tumour spread and surgical excision. Lancet 1986; 2(8514): 996-9.

3. Heald RJ, Ryall RD. Recurrence and survival after total mesorectal excision for rectal cancer. Lancet 1986; 1(8496): 1479-82.

4. Improved survival with preoperative radiotherapy in resectable rectal cancer. Swedish Rectal Cancer Trial. N Engl J Med 1997; 336(14): 980-7.

5. Kapiteijn E, Marijnen CA, Nagtegaal ID, et al. Preoperative radiotherapy combined with total mesorectal excision for resectable rectal cancer. N Engl J Med 2001; 345(9): 638-46.

6. van Gijn W, Marijnen CA, Nagtegaal ID, et al. Preoperative radiotherapy combined with total mesorectal excision for resectable rectal cancer: 12-year follow-up of the multicentre, randomised controlled TME trial. The lancet oncology 2011; 12(6): 575-82.

7. Landelijke Werkgroep Gastro Intestinale Tumoren. Richtlijn Colorectaal Carcinoom (versie 3.0). 16 April 2014. www.oncoline.nl. 
8. Maas M, Nelemans PJ, Valentini V, et al. Long-term outcome in patients with a pathological complete response after chemoradiation for rectal cancer: a pooled analysis of individual patient data. The lancet oncology 2010; 11(9): 835-44.

9. Vennix S, Pelzers L, Bouvy N, et al. Laparoscopic versus open total mesorectal excision for rectal cancer. The Cochrane database of systematic reviews 2014; 4: CD005200.

10. Marijnen CA, Kapiteijn E, van de Velde CJ, et al. Acute side effects and complications after shortterm preoperative radiotherapy combined with total mesorectal excision in primary rectal cancer: report of a multicenter randomized trial. J Clin Oncol 2002; 20(3): 817-25.

11. Lezoche G, Baldarelli M, Guerrieri M, et al. A prospective randomized study with a 5-year minimum follow-up evaluation of transanal endoscopic microsurgery versus laparoscopic total mesorectal excision after neoadjuvant therapy. Surgical endoscopy 2008; 22(2): 352-8.

12. Habr-Gama A, Perez RO, Nadalin W, et al. Operative versus nonoperative treatment for stage 0 distal rectal cancer following chemoradiation therapy: long-term results. Annals of surgery 2004; 240(4): 711-7; discussion 7-8.

13. Habr-Gama A, Perez RO, Proscurshim I, et al. Patterns of failure and survival for nonoperative treatment of stage c0 distal rectal cancer following neoadjuvant chemoradiation therapy. Journal of gastrointestinal surgery: official journal of the Society for Surgery of the Alimentary Tract 2006; 10(10): 1319-28; discussion 28-9.

14. Habr-Gama A, Gama-Rodrigues J, Sao Juliao GP, et al. Local recurrence after complete clinical response and watch and wait in rectal cancer after neoadjuvant chemoradiation: impact of salvage therapy on local disease control. International journal of radiation oncology, biology, physics 2014; 88(4): 822-8.

15. Appelt AL, Ploen J, Harling H, et al. High-dose chemoradiotherapy and watchful waiting for distal rectal cancer: a prospective observational study. The lancet oncology 2015; 16(8): 919-27.

16. Ayloor Seshadri R, Kondaveeti SS, Jayanand SB, et al. Complete clinical response to neoadjuvant chemoradiation in rectal cancers: can surgery be avoided? Hepatogastroenterology 2013; 60(123): 410-4.

17. Maas M, Beets-Tan RG, Lambregts DM, et al. Wait-and-see policy for clinical complete responders after chemoradiation for rectal cancer. J Clin Oncol 2011; 29(35): 4633-40.

18. Smith JD, Ruby JA, Goodman KA, et al. Nonoperative management of rectal cancer with complete clinical response after neoadjuvant therapy. Annals of surgery 2012; 256(6): 965-72.

19. Guillem JG, Chessin DB, Shia J, et al. Clinical examination following preoperative chemoradiation for rectal cancer is not a reliable surrogate end point. J Clin Oncol 2005; 23(15): 3475-9.

20. Hiotis SP, Weber SM, Cohen AM, et al. Assessing the predictive value of clinical complete response to neoadjuvant therapy for rectal cancer: an analysis of 488 patients. Journal of the American College of Surgeons 2002; 194(2): 131-5; discussion 5-6.

21. Kuo LJ, Chiou JF, Tai CJ, et al. Can we predict pathologic complete response before surgery for locally advanced rectal cancer treated with preoperative chemoradiation therapy? Int J Colorectal Dis 2012; 27(5): 613-21.

22. Maretto I, Pomerri F, Pucciarelli S, et al. The potential of restaging in the prediction of pathologic response after preoperative chemoradiotherapy for rectal cancer. Ann Surg Oncol 2007; 14(2): 455-61. 
23. Perez RO, Habr-Gama A, Pereira GV, et al. Role of biopsies in patients with residual rectal cancer following neoadjuvant chemoradiation after downsizing: can they rule out persisting cancer? Colorectal Dis 2012; 14(6): 714-20.

24. van der Paardt MP, Zagers MB, Beets-Tan RG, Stoker J, Bipat S. Patients Who Undergo Preoperative Chemoradiotherapy for Locally Advanced Rectal Cancer Restaged by Using Diagnostic MR Imaging: A Systematic Review and Meta-Analysis. Radiology 2013; 269(1): 101-12.

25. Vliegen RF, Beets GL, Lammering $G$, et al. Mesorectal fascia invasion after neoadjuvant chemotherapy and radiation therapy for locally advanced rectal cancer: accuracy of MR imaging for prediction. Radiology 2008; 246(2): 454-62.

26. Joye I, Deroose CM, Vandecaveye V, Haustermans K. The role of diffusion-weighted MRI and (18) F-FDG PET/CT in the prediction of pathologic complete response after radiochemotherapy for rectal cancer: a systematic review. Radiother Oncol 2014; 113(2): 158-65.

27. Adler J, Rahal K, Swanson SD, et al. Anti-tumor necrosis factor alpha prevents bowel fibrosis assessed by messenger RNA, histology, and magnetization transfer MRI in rats with Crohn's disease. Inflammatory bowel diseases 2013; 19(4): 683-90.

28. Adler J, Swanson SD, Schmiedlin-Ren P, et al. Magnetization transfer helps detect intestinal fibrosis in an animal model of Crohn disease. Radiology 2011; 259(1): 127-35.

29. Verseveld M, de Graaf EJ, Verhoef C, et al. Chemoradiation therapy for rectal cancer in the distal rectum followed by organ-sparing transanal endoscopic microsurgery (CARTS study). Br J Surg 2015; 102(7): 853-60.

30. Saclarides TJ, Speziale NJ, Drab E, Szeluga DJ, Rubin DB. Tumor angiogenesis and rectal carcinoma. Dis Colon Rectum 1994; 37(9): 921-6.

31. Sokmen S, Sarioglu S, Fuzun M, Terzi C, Kupelioglu A, Aslan B. Prognostic significance of angiogenesis in rectal cancer: a morphometric investigation. Anticancer research 2001; 21(6B): 4341-8.

32. George ML, Dzik-Jurasz AS, Padhani AR, et al. Non-invasive methods of assessing angiogenesis and their value in predicting response to treatment in colorectal cancer. Br J Surg 2001; 88(12): 1628-36.

33. Intven M, Reerink O, Philippens ME. Dynamic contrast enhanced MR imaging for rectal cancer response assessment after neo-adjuvant chemoradiation. J Magn Reson Imaging 2015; 41(6): 1646-53.

34. Lim JS, Kim D, Baek SE, et al. Perfusion MRI for the prediction of treatment response after preoperative chemoradiotherapy in locally advanced rectal cancer. Eur Radio/ 2012; 22(8): 1693700.

35. Oberholzer K, Menig M, Pohlmann A, et al. Rectal cancer: assessment of response to neoadjuvant chemoradiation by dynamic contrast-enhanced MRI. J Magn Reson Imaging 2013; 38(1): 119-26.

36. Tong T, Sun Y, Gollub MJ, et al. Dynamic contrast-enhanced MRI: Use in predicting pathological complete response to neoadjuvant chemoradiation in locally advanced rectal cancer. J Magn Reson Imaging 2015; 42(3): 673-80.

37. Zahra MA, Tan LT, Priest AN, et al. Semiquantitative and quantitative dynamic contrast-enhanced magnetic resonance imaging measurements predict radiation response in cervix cancer. International journal of radiation oncology, biology, physics 2009; 74(3): 766-73. 
38. DeVries AF, Kremser C, Hein PA, et al. Tumor microcirculation and diffusion predict therapy outcome for primary rectal carcinoma. International journal of radiation oncology, biology, physics 2003; 56(4): 958-65.

39. Kremser C, Trieb T, Rudisch A, Judmaier W, de Vries A. Dynamic T(1) mapping predicts outcome of chemoradiation therapy in primary rectal carcinoma: sequence implementation and data analysis. J Magn Reson Imaging 2007; 26(3): 662-71.

40. Sahani DV, Kalva SP, Hamberg LM, et al. Assessing tumor perfusion and treatment response in rectal cancer with multisection CT: initial observations. Radiology 2005; 234(3): 785-92. 


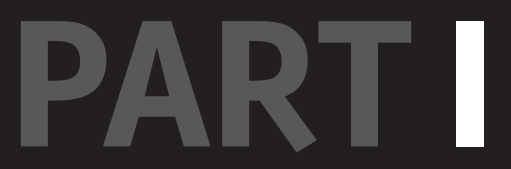

Organ preservation 



\section{CHAPTER 2}

Organ preservation in 100 clinical complete responders after neoadjuvant treatment for rectal cancer: long-term oncological and functional outcome

Authors:

M.H. Martens,

M. Maas,

L.A. Heijnen,

D.M.J. Lambregts,

J.W.A. Leijtens,

L.P.S. Stassen,

S.O. Breukink,

c. Hoff,

H.J. Belgers,

J. Melenhorst,

R.L.H. Jansen,

J. Buijsen,

A.G.M. Hoofwijk,

R.G.H. Beets-Tan,

G.L. Beets 


\section{Abstract \\ Objective}

The main aim was to confirm or refute the initially reported promising oncological results in a larger cohort of patients with a clinical (near) complete response. Second aim was to update the selection and inclusion criteria and to report the long-term functional outcome.

\section{Summary background data}

In patients with locally advanced rectal cancer and clinical complete response (CCR) after neoadjuvant chemoradiation, organ-preservation can be considered.

\section{Methods}

Between 2004 and 2014 organ-preservation was offered if response assessment with digital rectal examination, endoscopy, and state-of-the-art MRI showed a (near) complete clinical response (CCR). Watch-and-wait policy was offered for $\mathrm{CCR}$ and three options were offered for near CCR: Total mesorectal excision (TME), transanal endoscopic microsurgery (TEM), or a second assessment after 3 months. Follow-up included endoscopy and MRI 3-monthly during first year, and 6-monthly thereafter. Long-term outcome was assessed with KaplanMeier curves. Functional outcome was assessed two years after inclusion with the Vaizeyscore.

\section{Results}

After informed consent 100 patients were included with median follow-up of 37.9 months. Sixty-one had CCR at initial response assessment. Thirty-nine had near CCR, of whom 24 developed a cCR at the second assessment and 15 patients underwent TEM (9 ypT0, 1 ypT1, 5 ypT2). Fifteen patients developed local regrowth (12 luminal, 3 nodal), all salvageable and within 25 months. Five patients developed metastases (3 lung, 1 liver and lung, 1 peritoneal) and four patients died. Three-year local regrowth free survival was $84 \%$, distant metastases free survival $97 \%$, disease free survival $81 \%$, and overall survival $96 \%$. Mean Vaizey-score was significantly better in watch-and-wait patients compared to TEM (3.0 vs $9 \cdot 7, p=0 \cdot 002)$.

\section{Conclusions}

Organ-preservation appears oncologically safe for selected rectal cancer patients with a cCR after neoadjuvant chemoradiation. Near complete responders can be reassessed after 3 months or undergo TEM. 


\section{Introduction}

The standard treatment for patients with a locally advanced rectal cancer is neoadjuvant chemoradiation followed by total mesorectal excision (TME) after a 6-10 week interval. In up to $15-20 \%$ of the patients, no residual tumour is found at histopathology ${ }^{1}$. In 2004, HabrGama et al. suggested that surgery may be omitted in a selected patient group with a clinical complete response $(\mathrm{CCR})^{2}$. Although initially this concept resulted in considerable scepticism, there is now an increasing interest in organ-preservation in patients with a clinical complete response. Both omission of surgery with a watch-and-wait regimen ${ }^{3-6}$ and transanal endoscopic microsurgery (TEM) of the residual scar ${ }^{7-9}$ have shown promising outcomes in relatively small series.

In 2011 we reported a pilot study on watch-and-wait (W\&W) policy in 21 patients with a clinical complete response after neoadjuvant chemoradiation, with an important role for MRI in the selection and follow-up of patients ${ }^{4}$. In this pilot study, the 2-year disease-free survival was $89 \%$ and the overall survival $100 \%$. As expected, the functional outcomes were also better when compared to complete responders that underwent TME. However, the number of patients was limited and the mean follow-up was relatively short. Since then we have experienced a high interest of both clinicians and patients, and we have continued to offer organ-preserving treatment. The selection criteria were slightly widened based on the initial favourable experience and TEM was performed for patients with a very good, but not complete response.

The main aim of this study was to confirm or refute the initially promising oncological results in a larger cohort of patients with a clinical complete or near complete response after neoadjuvant chemoradiation for rectal cancer. Secondary aims were to update the selection and inclusion criteria and to report the long-term functional outcome.

\section{Material and Methods}

\section{Patient selection}

Between 2004 and 2014, patients who were treated with neoadjuvant chemoradiotherapy in standard patient care and presented with a clinical complete response at restaging, were offered an organ-preserving treatment. Patient were either primarily diagnosed and treated in our center or were referred to our center for clinical response assessment and evaluation of eligibility for organ-preservation after completion of neoadjuvant treatment in another center. Organ-preservation was offered in a prospective cohort study, approved by the local IRB and registered in clinicaltrials.gov since 2009 (NCT00939666 and NCT02278653). All patients provided informed consent. 
Table 1. Criteria for a clinical complete response after neoadjuvant therapy.

\begin{tabular}{|c|c|c|}
\hline Modality & Criteria for clinical complete response & $\begin{array}{l}\text { Criteria for clinical near complete } \\
\text { response }\end{array}$ \\
\hline DRE & $\begin{array}{l}\text { No palpable tumor, when initially } \\
\text { palpable with DRE }\end{array}$ & Small palpable flexible ridge \\
\hline Endoscopy & No residual tumor AND white scar & $\begin{array}{l}\text { Small residual erythematous ulcer OR } \\
\text { Irregular wall thickening }\end{array}$ \\
\hline \multirow{3}{*}{ MRI } & $\begin{array}{l}\text { Standard T2-weighted MRI } \\
\text { - } \quad \text { Substantial downsizing with no } \\
\text { residual tumor OR } \\
\text { - } \quad \text { Residual fibrosis OR } \\
\text { - } \quad \text { Residual wall thickening due to } \\
\quad \text { edema } \\
\text { AND no suspicious lymph nodes }\end{array}$ & $\begin{array}{l}\text { Standard T2-weighted MRI } \\
\text { - Obvious downstaging with residual } \\
\text { fibrosis, but heterogeneous or } \\
\text { irregular aspect and signal OR } \\
\text { - Obvious downstaging of lymph nodes, } \\
\text { but remaining node }(\mathrm{s}) \geq 5 \mathrm{~mm}\end{array}$ \\
\hline & $\begin{array}{l}\text { Diffusion-weighted MRI } \\
\text { Low signal on high b-value }\end{array}$ & $\begin{array}{l}\text { Diffusion-weighted } \mathrm{MRI}^{*} \\
\text { Small focal area of high signal on high b- } \\
\text { value }\end{array}$ \\
\hline & $\begin{array}{l}\text { Gadofosveset-enhanced MRI }{ }^{*} \\
\text { No suspicious lymph nodes }\end{array}$ & $\begin{array}{l}\text { Gadofosveset-enhanced MRI } \\
\text { Obvious downstaging of lymph nodes, } \\
\text { but remaining node }(\mathrm{s}) \geq 5 \mathrm{~mm} \text { without } \\
\text { malignant enhancement pattern }\end{array}$ \\
\hline Histopathology & $\begin{array}{l}\text { Negative biopsies from scar (biopsy not } \\
\text { mandatory) }\end{array}$ & Dysplastic changes \\
\hline
\end{tabular}

¥ Patients had a clinical near complete response if they missed only one or two criteria of a clinical complete response, but matched the other criteria for a clinical near complete response. * available since 2006, DRE = digital rectal exam; MRI = magnetic resonance imaging

Inclusion criteria were [1] rectal cancer without distant metastasis, [2] neoadjuvant treatment (standard chemoradiation of $28 \times 1.8 \mathrm{~Gy}$ combined with $2 \times 825 \mathrm{mg} / \mathrm{m}^{2}$ capecitabine, or $5 \times 5 \mathrm{~Gy}$ with a long interval to surgery), and [3] a clinical complete or near complete response. The response was evaluated approximately 8 weeks after completion of neoadjuvant treatment with digital rectal examination (DRE), MRI, and endoscopy. Criteria for a clinical complete response were described previously ${ }^{4}$ (table 1 ). Eligible patients with a clinical complete response were offered the watch-and-wait policy as an alternative to standard TME. Patients were specifically informed of the experimental nature and the potentially increased risk of this alternative treatment in a shared-decision process. Figure 1 represents a typical example of the MRI and endoscopic images of a patient eligible for the watch-and-wait policy. Some patients had a near complete response at initial response assessment, defined as a very good response that did not meet all criteria for a clinical complete response, for example a small red lesion at endoscopy, or equivocal lymph nodes on MRI (see table 1). Figure 2 shows an example the MRI and endoscopic images of a patient with a near complete response. These patients were offered, additional to the standard TME the following two alternative options: [1] Transanal Endoscopic Microsurgery (TEM), or [2] postponing the decision between W\&W and TME until a second assessment after 3 months. Patients that met all the criteria for CCR at this second assessment, were offered watch-and-wait. All other patients were referred for TME. 

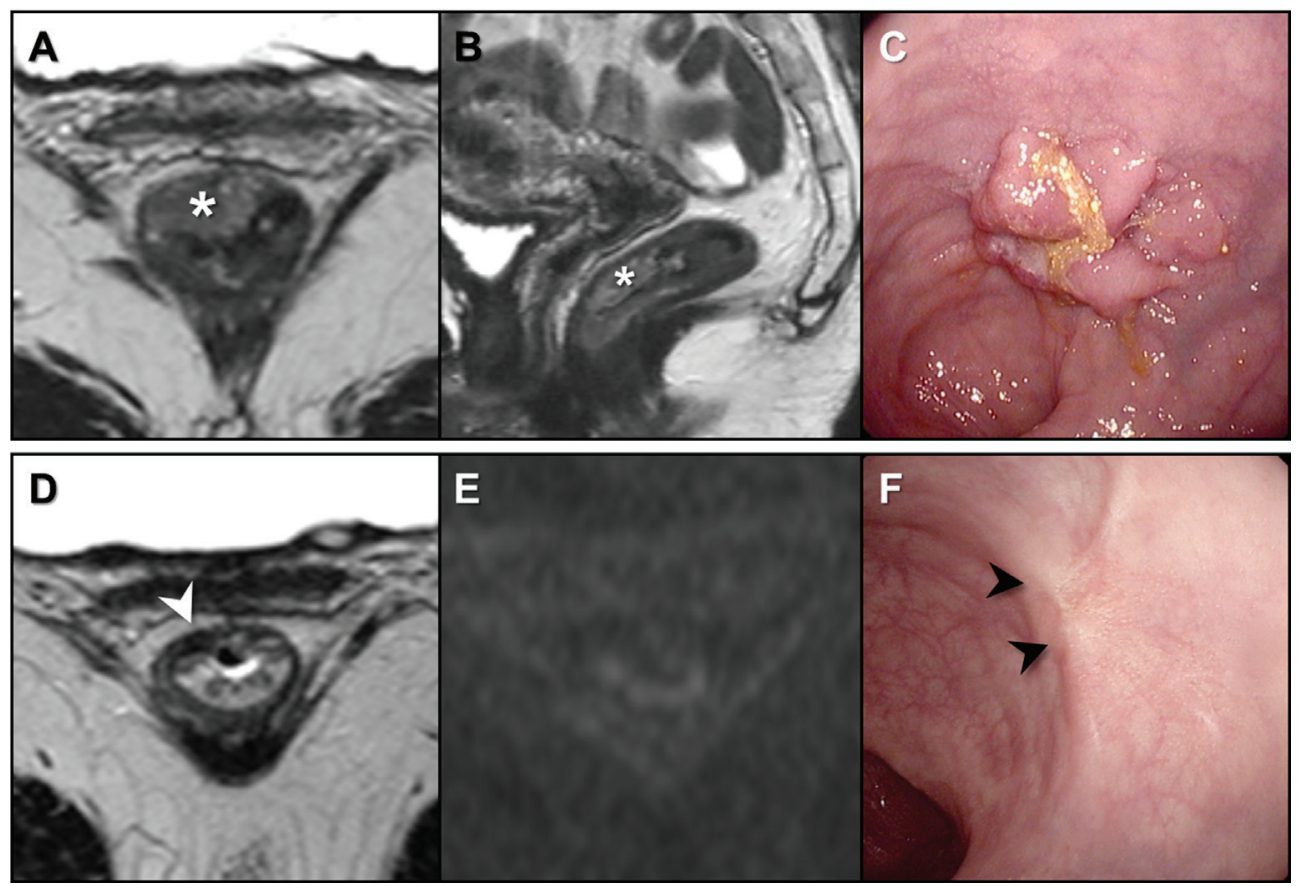

Figure 1. Images of a patient with a clinical complete response at the initial response assessment.

Axial (A) and saggital (B) T2-weighted MRI of a distal cT2N0 tumor before treatment (indicated with *) and (C) endoscopic image of primary tumor. (D) Axial T2-weighted MRI eight weeks post-chemoradiation, with the white arrowhead indicating residual fibrosis. (E) Diffusion-weighted MRI without any high signal, suggesting complete response. (F) Endoscopy with a typical white scar (black arrow heads).

\section{Follow-up}

Follow-up included the standard follow-up according to national guidelines, with imaging of the chest and liver, CEA, and out-patient clinic visits for a period of 5 years. For the organpreservation specific follow-up, patients additionally underwent MRI and sigmoidoscopy every 3 months during the first year, and every 6 months until 5 years after inclusion ${ }^{4}$ (See Table 2).

\section{Functional outcome}

Complications after TEM were recorded and classified according to Dindo et al. ${ }^{10}$ Patients with a follow-up of at least 2 years were approached to participate in a questionnaire to assess the presence of faecal incontinence. Functional outcome was measured with the Vaizey score ${ }^{11}$, consisting of 7 questions with a total score ranging between 0 (no incontinence) and 24 (major incontinence). All scores $\geq 12$ are considered major incontinence. 

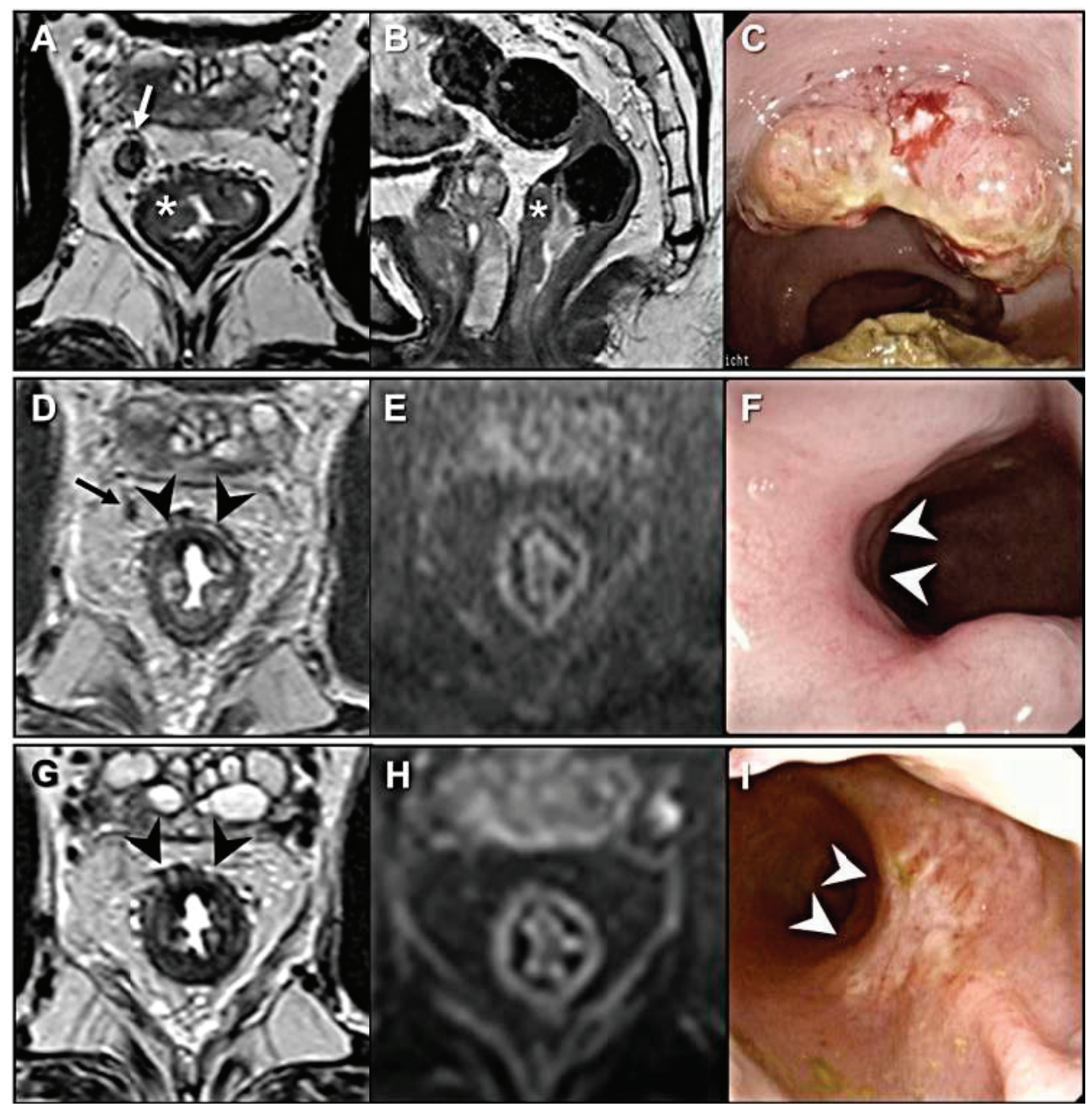

Figure 2. Images of a patient with a near complete response at initial assessment and clinical complete response after a reassessment 3 months.

Axial (A) and saggital (B) T2-weighted MRI of distal cT3 tumor (indicated with *) with evident malignant lymph node (white arrow) before treatment and (C) endoscopic image of primary tumor. (D) Axial T2-weighted MRI at initial response assessment 16 weeks post-chemoradiation, with the black arrow indicating a residual lymph node, potentially malignant. Black arrow heads indicate residual fibrosis. (E) Diffusion-weighted MRI without any high signal, suggesting complete response. (F) Endoscopy with a small superficial erythematous ulcer (white arrow heads). (G) Axial T2-weighted MRI 3 months later, the lymph node has disappeared. Black arrow heads indicate residual fibrosis. (H) Diffusion-weighted MRI without high signal. (I) Typical white scar (white arrow heads) visible on endoscopy. 


\section{Statistical analyses}

Statistical analyses were performed using the Statistical Package for the Social Sciences (SPSS version 20.0, Inc., Chicago, Illinois, USA). Functional outcome scores were compared between W\&W and TEM patients with a Mann-Whitney $U$ test. Local regrowth free survival (LRFS), distant metastasis free survival (DMFS), disease free survival (DFS) and overall survival (OS) were estimated with Kaplan-Meier curves and were compared between different groups using the log-rank test.

Local regrowth was defined as tumour regrowth in the lumen or in a local lymph node, DMFS as the absence of distant metastases, DFS as the absence of local regrowth, distant metastasis, and death from any cause, and OS as the absence of death from any cause. Duration of follow-up was calculated from the start of treatment (first day of radiotherapy) to the event of interest or the last follow-up date. A p-value $\leq 0.05$ was considered statistically significant.

Table 2. Follow-up schedule for patients with organ-preserving therapy

\begin{tabular}{|c|c|c|c|c|c|}
\hline Year & CEA & DRE & $\begin{array}{c}\text { CT for distant } \\
\text { staging }\end{array}$ & Endoscopy & MRI \\
\hline $\mathbf{1}$ & $4 \mathrm{x}$ & $4 \mathrm{x}$ & $2 \mathrm{x}$ & $4 \mathrm{x}$ & $4 \mathrm{x}$ \\
\hline $\mathbf{2}$ & $4 \mathrm{x}$ & $2 \mathrm{x}$ & $1 \mathrm{x}$ & $2 \mathrm{x}$ & $2 \mathrm{x}$ \\
\hline 3 & $4 \mathrm{x}$ & $2 \mathrm{x}$ & $1 \mathrm{x}$ & $2 \mathrm{x}$ & $2 \mathrm{x}$ \\
\hline $\mathbf{4}$ & $2 \mathrm{x}$ & $2 \mathrm{x}$ & $1 \mathrm{x}$ & $2 \mathrm{x}$ & $2 \mathrm{x}$ \\
\hline 5 & $2 \mathrm{x}$ & $2 \mathrm{x}$ & $1 \mathrm{x}$ & $2 \mathrm{x}$ & \\
\hline
\end{tabular}

Marked in grey indicates the additional follow-up compared to standard follow-up after total mesorectal excision.

$\mathrm{CEA}=$ carcinoembryogenic antigen; $\mathrm{DRE}=$ digital rectal exam; $\mathrm{MRI}$ = magnetic resonance imaging; $\mathrm{CT}=$ computed tomography

\section{Results}

A total of 105 patients was considered for organ-preserving treatment of whom eventually 100 were included in the programme. Sixty-one patients had a CCR at the first assessment and were immediately included in the watch-and-wait policy (W\&W). The other 44 patients had a near CCR at initial assessment, of whom 15 patients underwent an immediate TEM, while the remaining 29 patients underwent a second assessment after 3 months. In the 15 patients who underwent a TEM, histopathology showed a ypT0 in 9/15 patients (60\%), ypT1 in one patient (7\%), ypT2 in and 5/15 patients (33\%). None of the patients with a ypT1 or ypT2 underwent immediate completion TME. For the 29 patients who were reassessed after 3 months, the most common finding of a near complete response at initial response assessment was an inconclusive endoscopy such as a small red lesion or ulcer, while MRI showed a cCR (11/29, 40\%). Other findings were equivocal lymph nodes (5/29, 17\%), residual high signal on DWI but fibrosis on T2-weighted MRI with cCR at endoscopy (3/29, 10\%), 
residual tumour on T2-weighted but no signal on DWI with cCR at endoscopy (1/29, 3\%), or near complete response both on endoscopy and MRI (9/29, 31\%). Reassessment after 3 months showed a typical complete response on both MRI and endoscopy in 24 patients, who all chose for the watch-and-wait policy and can be considered delayed inclusions. Five patients were judged not to have a cCR at this second assessment and were referred for TME: one patient had a pCR, the remaining 4 patients had residual tumour remnants. These five patients with an incomplete clinical response were excluded from further analyses. See the flowchart for details on the selection and inclusion process (figure 3).

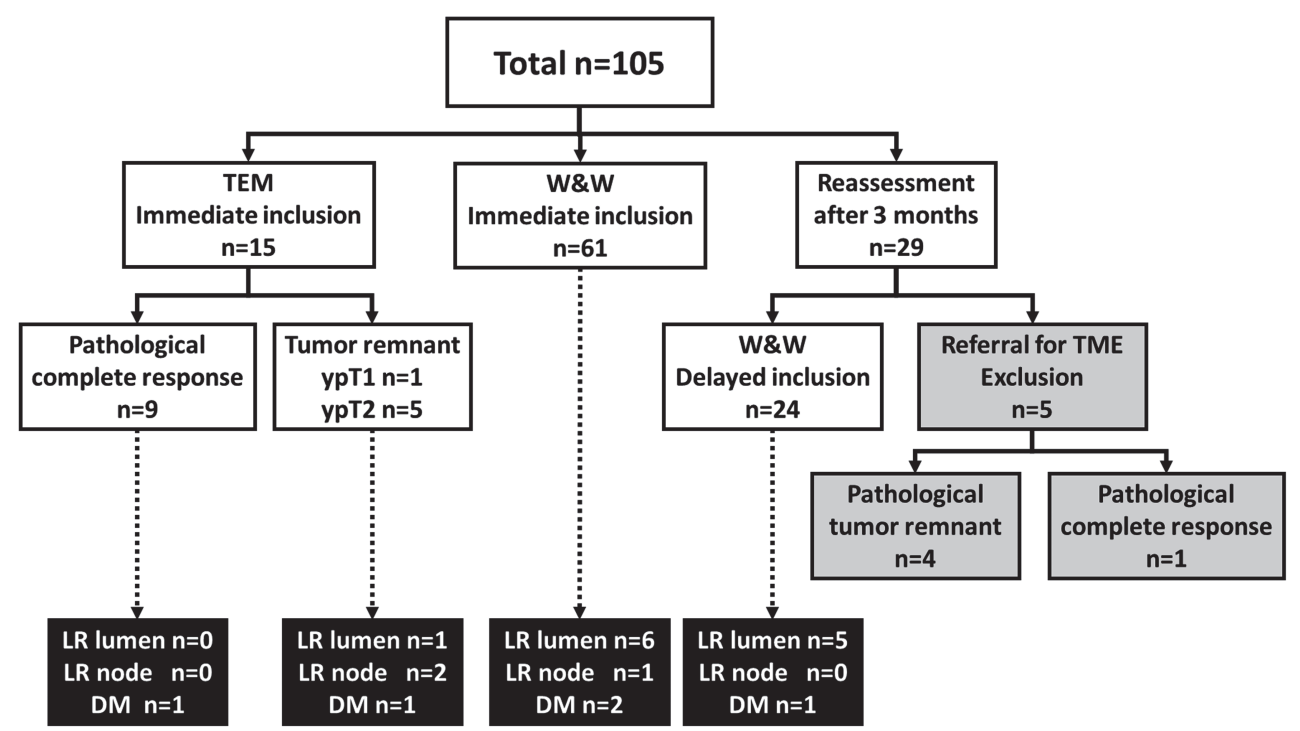

Figure 3. Flowchart of patient selection and inclusion.

TEM = transanal endoscopic microsurgery; W\&S = wait-and-see; TME = total mesorectal excision; LR = local regrowth; DM = distant metastasis

Overall 100 patients were included for organ-preservation. Sixty-seven patients were male $(67 \%)$ and the mean age was 63.2 \pm 10.5 years. Median follow-up was 37.9 months (range 11.7-119.7), and 52 patients (52\%) had a follow-up of at least 3 years. Detailed patientand treatment characteristics are given in table 3. Of the included 100 patients, 24 were diagnosed and treated in our center, constituting 17\% (24/141) of all patients treated with chemoradiation in our center. The remaining 76 included patients were primarily treated in another centre and referred to our center specifically for organ-preservation.

\section{Oncological outcome}

Fifteen of the 100 patients treated with an organ-preserving treatment developed a local regrowth within the lumen $(n=12)$ or in a lymph node $(n=3)$. All luminal regrowths were detected with endoscopy, in four patients it was also visible on MRI (in 3 patients visible on 
Table 3. Patient characteristics

\begin{tabular}{|c|c|c|c|c|}
\hline Parameter & Categories & $\begin{array}{l}\text { Total } \\
(n=100)\end{array}$ & $W \& S(n=85)$ & $\begin{array}{l}\text { TEM } \\
(n=15)\end{array}$ \\
\hline Age $[$ mean $( \pm S D)]$ & Years & $63.2( \pm 10.5)$ & $62.7( \pm 10.6)$ & $65.8( \pm 9.8)$ \\
\hline $\operatorname{Sex}[n(\%)]$ & $\begin{array}{l}\text { Male } \\
\text { female }\end{array}$ & $\begin{array}{l}67(67 \%) \\
33(33 \%)\end{array}$ & $\begin{array}{l}56(66 \%) \\
29(34 \%) \\
\end{array}$ & $\begin{array}{l}11(73 \%) \\
4(27 \%)\end{array}$ \\
\hline Clinical T-stage [n (\%)] & $\begin{array}{l}\text { CT1-2 } \\
\text { CT3 } \\
\text { cT4 }\end{array}$ & $\begin{array}{l}25(25 \%) \\
67(67 \%) \\
8(8 \%) \\
\end{array}$ & $\begin{array}{l}22(26 \%) \\
55(65 \%) \\
8(9 \%) \\
\end{array}$ & $\begin{array}{l}3(20 \%) \\
12(80 \%) \\
-\end{array}$ \\
\hline $\begin{array}{l}\text { Distance from anorectal verge } \\
\text { [n (\%)] }\end{array}$ & $\begin{array}{l}0-5 \mathrm{~cm} \\
5-10 \mathrm{~cm} \\
10-15 \mathrm{~cm}\end{array}$ & $\begin{array}{l}79(79 \%) \\
18(18 \%) \\
3(3 \%) \\
\end{array}$ & $\begin{array}{l}70(82 \%) \\
12(14 \%) \\
3(4 \%) \\
\end{array}$ & $\begin{array}{l}9(60 \%) \\
6(40 \%) \\
-\end{array}$ \\
\hline Clinical N-stage [n (\%)] & $\begin{array}{l}\mathrm{CN} 0 \\
\mathrm{cN} 1 \\
\mathrm{cN} 2\end{array}$ & $\begin{array}{l}26(26 \%) \\
41(41 \%) \\
33(33 \%)\end{array}$ & $\begin{array}{l}20(24 \%) \\
36(42 \%) \\
29(34 \%)\end{array}$ & $\begin{array}{l}6(40 \%) \\
5(33 \%) \\
4(27 \%)\end{array}$ \\
\hline Neoadjuvant treatment [n (\%)] & $\begin{array}{l}\text { 50.4Gy + Capecitabine } \\
5 \times 5 G y+\text { CAPOX } \\
5 \times 5 G y+\text { Rapamycine } \\
5 \times 5 G y+\text { interval }\end{array}$ & $\begin{array}{l}95(95 \%) \\
2(2 \%) \\
2(2 \%) \\
1(1 \%) \\
\end{array}$ & $\begin{array}{l}83(98 \%) \\
2(2 \%) \\
- \\
- \\
\end{array}$ & $\begin{array}{l}12(80 \%) \\
- \\
2(13 \%) \\
1(7 \%) \\
\end{array}$ \\
\hline $\begin{array}{l}\text { "Adjuvant" chemotherapy } \\
\text { (CAPOX) [n (\%)]" }\end{array}$ & $\begin{array}{l}\text { Yes } \\
\text { No } \\
\text { Incomplete }\end{array}$ & $\begin{array}{l}34(34 \%) \\
60(60 \%) \\
6(6 \%) \\
\end{array}$ & $\begin{array}{l}32(38 \%) \\
48(56 \%) \\
5(6 \%)\end{array}$ & $\begin{array}{l}2(13 \%) \\
12(80 \%) \\
1(7 \%) \\
\end{array}$ \\
\hline $\begin{array}{l}\text { Interval between last RT } x \text { and } \\
\text { assessment post-CRT } \\
\text { [median(IQR)] }\end{array}$ & Weeks & $\begin{array}{l}10.0 \\
(3.3-16.7)\end{array}$ & $\begin{array}{l}10.0 \\
(3.1-16.9)\end{array}$ & $\begin{array}{l}10.7 \\
(5.8-16.6)\end{array}$ \\
\hline Timing of inclusion [n (\%)] & $\begin{array}{l}\text { First assessment post-CRT } \\
\pm 3 \text { months re-assessment }\end{array}$ & $\begin{array}{l}61(61 \%) \\
24(24 \%) \\
\end{array}$ & $\begin{array}{l}61(72 \%) \\
24(28 \%) \\
\end{array}$ & n.a. \\
\hline Pathology [n (\%)]^ & $\begin{array}{l}\text { урт0 } \\
\text { урT1 } \\
\text { урт2 }\end{array}$ & n.a. & n.a. & $\begin{array}{l}9(60 \%) \\
1(7 \%) \\
5(33 \%) \\
\end{array}$ \\
\hline $\begin{array}{l}\text { Postoperative complication } \\
\text { according to Dindo } \\
\text { classification [n (\%)] }\end{array}$ & $\begin{array}{l}\text { No complications } \\
\text { I } \\
\text { II } \\
\text { IIIa } \\
\text { IVa }\end{array}$ & n.a. & n.a. & $\begin{array}{l}2(13 \%) \\
7(47 \%) \\
4(27 \%) \\
1(7 \%) \\
1(7 \%)\end{array}$ \\
\hline
\end{tabular}

* Adjuvant chemotherapy was offered according to our national guidelines, $\wedge$ Pathology after TEM, n.a. = not applicable

both T2W and DWI, in one patient only on DWI). All lymph node regrowths were detected with MRI. All regrowths occurred within 25 months of follow-up. See Figure 4 for MRI and endoscopy images of a luminal regrowth after 10 months of follow-up. Figure 5 showes MRI and endoscopy images of a nodal regrowth. In two patients with a local regrowth, distant metastasis were observed simultaneously, and the treatment was primarily aimed at the metastases with a continued control of the local situation. 


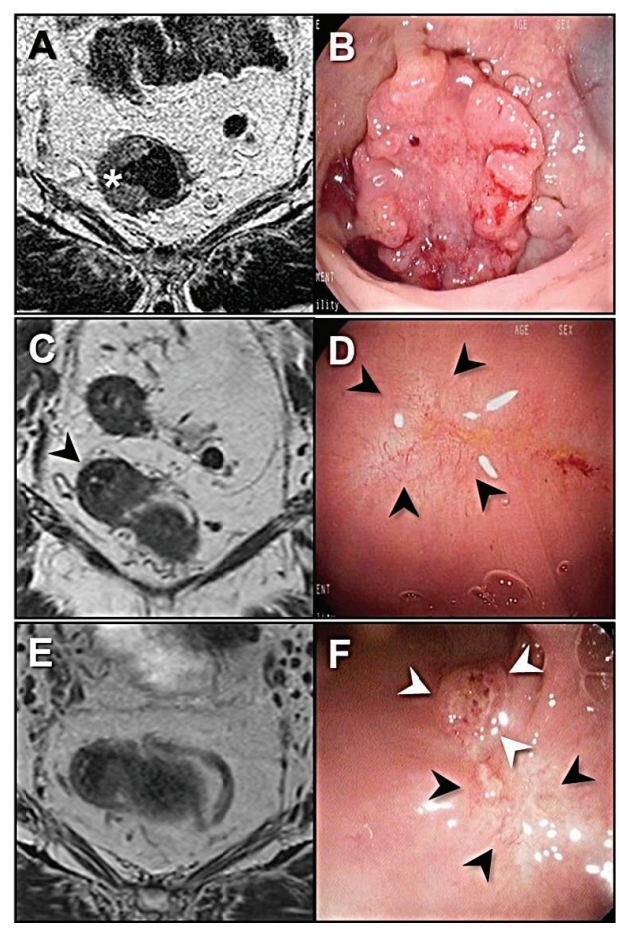

Figure 4. Images of a patient included in wait-and-see group after initial response assessment and who developed a luminal regrowth after 10 months of follow-up.

(A) Axial T2-weighted MRI of primary CT3N1 tumor (indicated with *). (B) Endoscopy of primary tumor. (C) Axial T2-weighted MRI at initial assessment nine weeks after completion of chemoradiation, black arrowhead indicates a small fibrotic area. (D) White scar at endoscopy typical for CCR. (E) Axial T2-weighted MRI after 10 months of follow-up without any sign of regrowth. (F) On endoscopy the white scar is still visible (black arrowheads), but adjacent there is a nodular lesion (white arrowheads). Biopsy showed invasive adenocarcinoma and patient was referred for resection (ypT2N0).

All but one of 13 isolated local regrowths without synchronous metastasis were detected when small and were easily amenable to salvage therapy with a surgical procedure that was not more extensive than it would have been originally. One patient required a pelvic exenteration for a regrowth after a TEM for a small remaining ypT2 residual anterior lesion. At present, all 13 patients remained free of further local recurrence. In addition to the two patients with simultaneous local and distant recurrence, three other patients developed metastases in lungs, both liver and lung, or peritoneum. Two patients died of metastatic disease and two other patients of unrelated causes. Table 4 gives a complete overview of all regrowths, distant metastases, and causes of death. The cumulative probability of 3-year local regrowth free survival was $84 \%$, distant metastasis free survival 97\%, disease free survival 81\%, and overall survival was 96\% (Figure 6). 
Of the 15 patients with a local regrowth, 3 occurred in the TEM-group, all in patients with ypT2 residual tumour. The 3-year LRFS for the TEM-group versus the W\&W-group was $80 \%$ vs $85 \%(p=0.60)$, DMFS and OS were not statistically different between the two groups (Table 5). The LRFS for watch-and-wait patients who were included after a second assessment was $77 \%$, compared to $88 \%$ for the patients that were included at the first assessment $(p=0.22)$. No significant difference between the two groups was found with regard to DMFS (96\% vs $98 \%, p=0.73)$ and OS (96\% vs 96\%, $p=0.95)$.

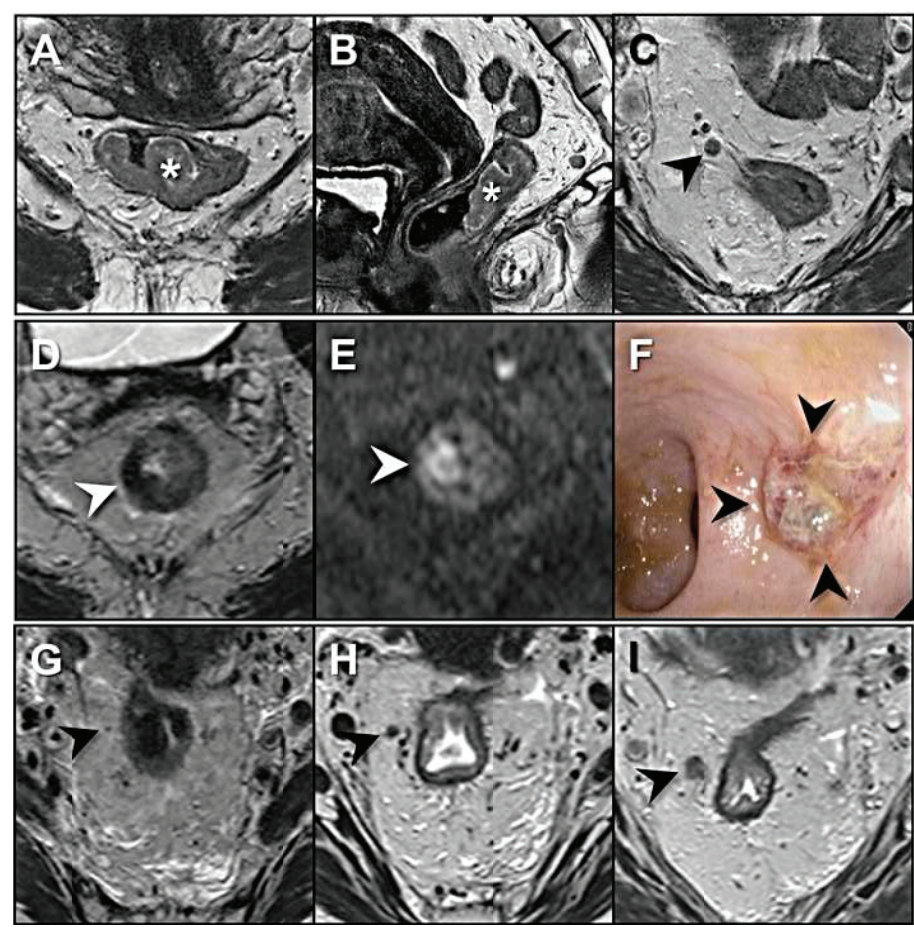

Figure 5. Images of a patient with a near complete response who underwent TEM, with histopathology showing yрT2 residual tumor. Six months later a nodal regrowth was detected.

(A) Axial (B) and saggital T2-weighted MRI of primary cT3N2 tumor (indicated with *). (C) Axial T2-weighted MRI with black arrowhead indicating malignant lymph node. (D) Axial T2-weighted MRI at initial response assessment 16 weeks post-chemoradiation, black arrowhead indicates residual fibrosis. (E) Diffusion-weighted MRI with focal high signal indicating residual tumor. (F) Endoscopy shows a small residual tumor. Patient was referred for TEM and histopathology showed ypT2 tumor. (G) Axial T2-weighted MRI at initial response assessment with evident regression of the lymph node. (H) Three months post-TEM the lymph node appear larger. (I) Six months post-TEM this was diagnosed as a nodal regrowth. Unfortunately, this patient had simultaneous multiple pulmonary metastasis without curative options. 


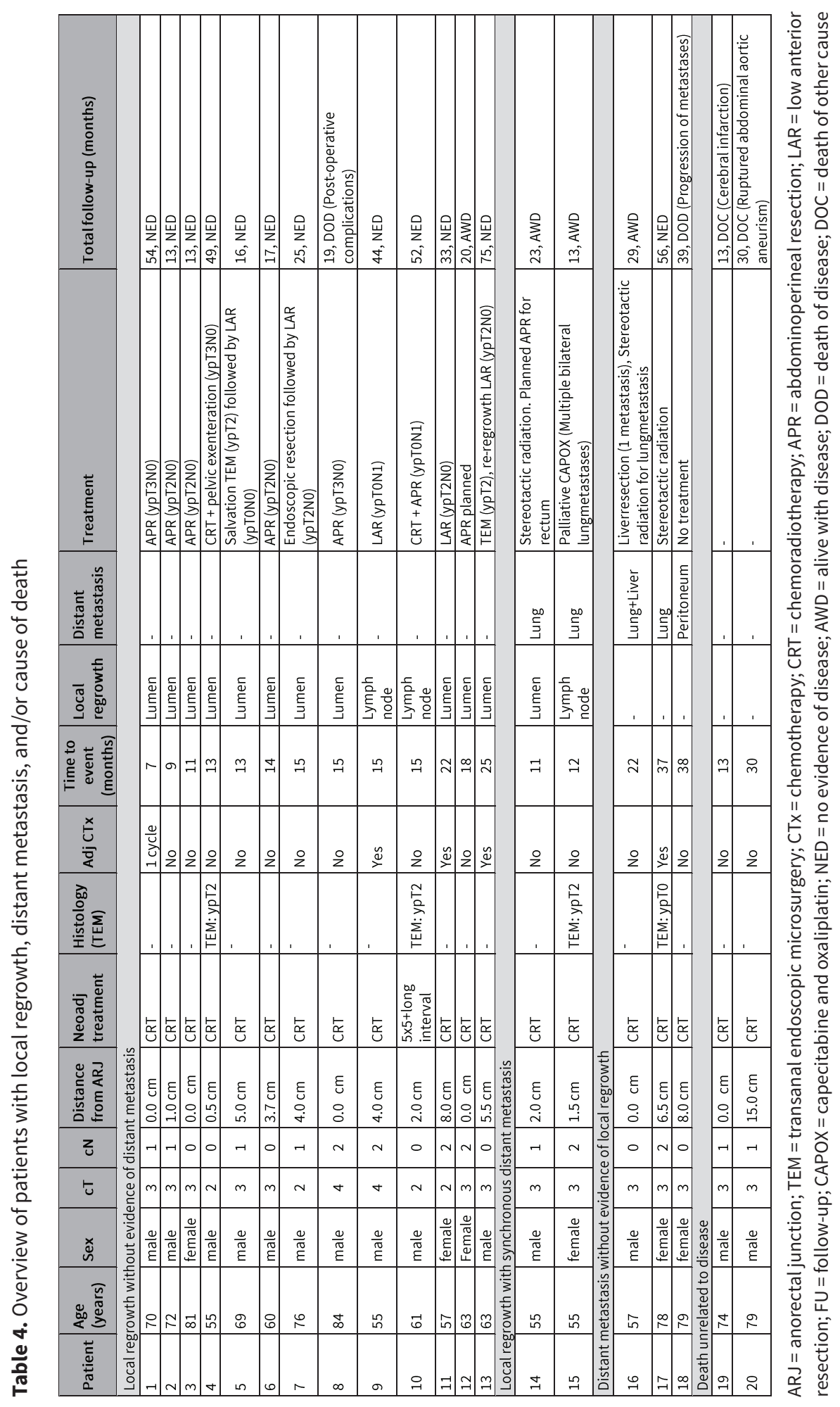



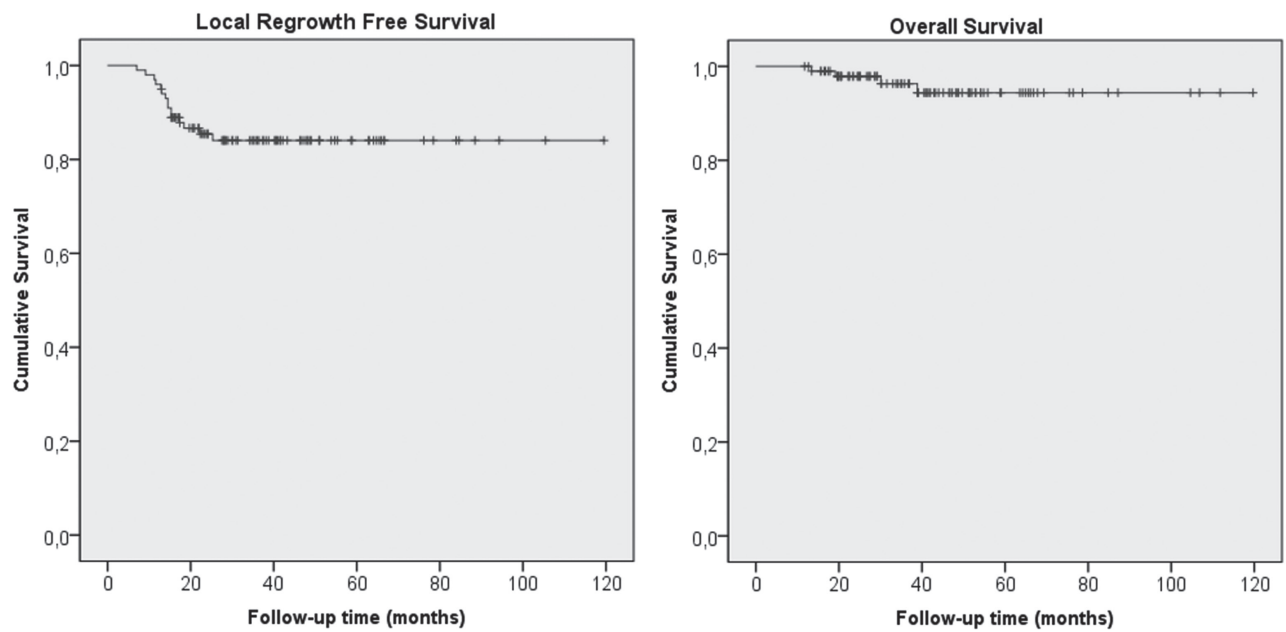

Figure 6. Local regrowth free survival and overall survival for the total of 100 patients in an organ-preserving treatment.

Table 5. Three-year survival for W\&W compared to TEM, and immediate inclusion compared to reassessment after 3 months.

\begin{tabular}{|l|l|l|l|l|}
\hline $\begin{array}{l}\text { 3-year } \\
\text { survival }\end{array}$ & W\&W vs TEM & p-value & $\begin{array}{l}\text { Immediate inclusion vs } \\
\text { reassessment }\end{array}$ & p-value \\
\hline LRFS & $85 \%$ vs $80 \%$ & 0.60 & $88 \%$ vs $77 \%$ & 0.22 \\
\hline DMFS & $97 \%$ vs $93 \%$ & 0.18 & $98 \%$ vs $96 \%$ & 0.73 \\
\hline DFS & $81 \%$ vs $80 \%$ & 0.60 & $83 \%$ vs $74 \%$ & 0.27 \\
\hline OS & $96 \%$ vs $100 \%$ & 0.36 & $96 \%$ vs $96 \%$ & 0.95 \\
\hline
\end{tabular}

\section{Functional outcome}

Forty-six patients received the questionnaire of whom 29 completed the questionnaires (22 W\&W, 7 TEM, response rate 63\%). The total Vaizey score of W\&W-patients (mean 3.4 \pm 3.9 ) Was significantly better than TEM-patients (mean 9·7 $55 \cdot 1, p=0 \cdot 003$ ). Patients in the watch-andwait policy scored better on three items: incontinence for liquid stool, incontinence for gas, and the need for a pad or plug. See figure 7 for detailed results. Only one W\&W-patient $(1 / 22$, $5 \%$ ) suffered from major incontinence (score $\geq 12$ ) compared to three patients in the TEM group (3/7, 43\%). All but two TEM-patients (13/15) had post-operative complications, 6 of whom with a Dindo classification of II or higher. Most long-term complications were peri-anal and abdominal pain (54\%). 


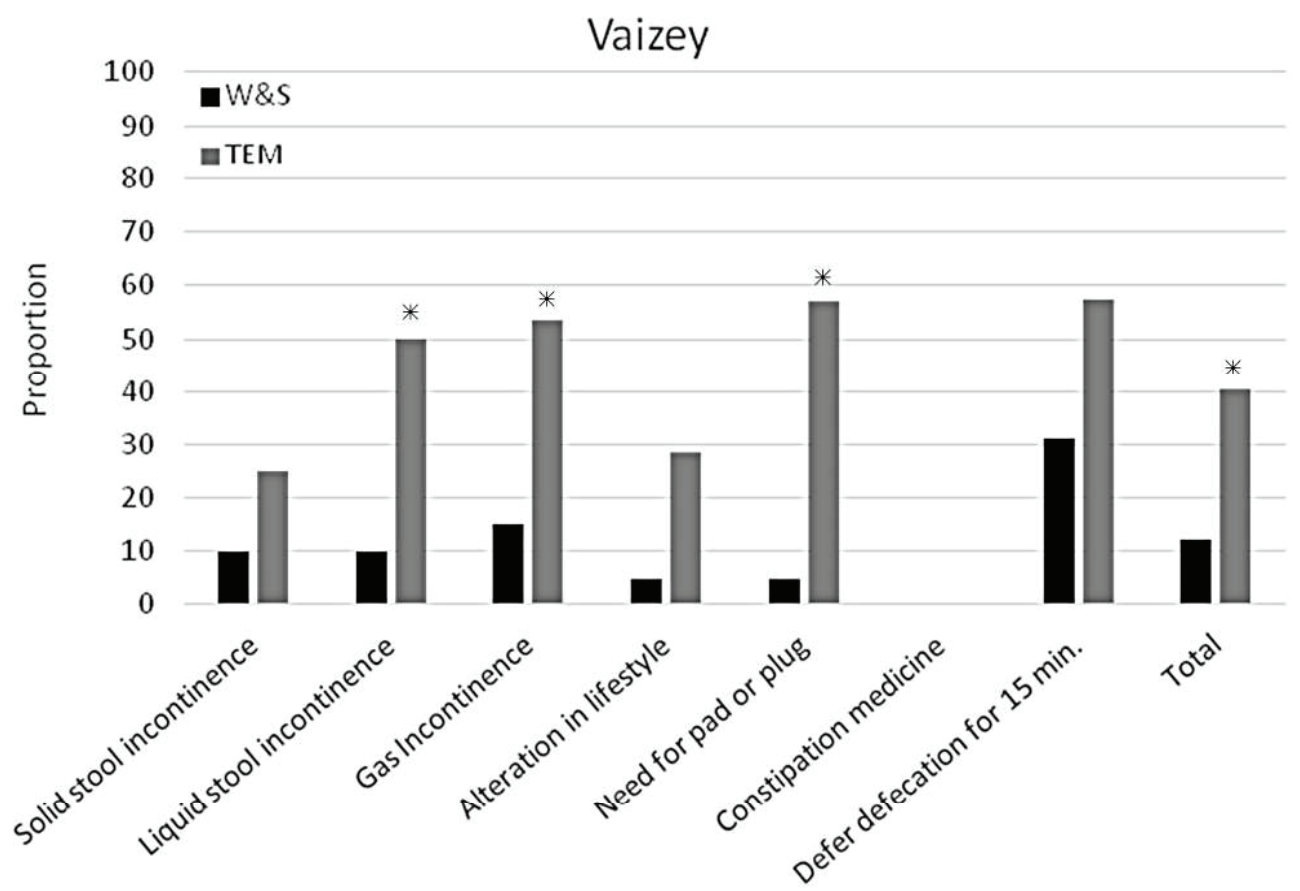

Figure 7. Functional outcome based on Vaizey score. A higher score indicates a poorer result.

* Indicates that the difference was statistically significant.

\section{Discussion}

The main conclusion is that organ-preserving treatment for (near) complete responders after CRT for locally advanced rectal cancer is feasible and results in a high 3-year diseasefree survival of $81 \%$ and a high overall survival of $96 \%$. This study suggests that in a careful program of organ-preservation there is little or no oncological disadvantage for selected patients with a (near) clinical complete response.

There is a legitimate concern that organ-preservation in rectal cancer could negate the improvements in oncological outcome that have been achieved by optimal use of a good surgical TME technique and (neo)adjuvant treatment. The concern is that the increased number of local recurrences will lead to a decreased survival because some may not be amenable to salvage therapy and because some may cause metastases later. In the present study only one of the hundred patients was disadvantaged by the organ-preservation approach because a pelvic exenteration was required to control the local regrowth. This occurred after a TEM for a ypT2 tumour, where we now strongly recommend immediate completion surgery. We have not yet seen an unresectable local regrowth, and encountered only one local recurrence after salvage TEM and no local recurrence after salvage TME surgery. In two patients with a local regrowth, systemic disease was diagnosed simultaneously, and there were no patients with systemic disease diagnosed after the local regrowth. Given this timeframe, it is unlikely that the regrowth was the source of the metastases, although follow- 
up after local regrowth is relatively short in the majority of the patients (Table 3) Even more, three patients developed distant metastasis without evidence of a local regrowth. The recent report of Habr-Gama et al ${ }^{12}$ on watch-and-wait in 90 patients with a median follow-up of 60 months showed a local regrowth in 31\%, higher than the 14\% in the present study. In that study the majority was easily amenable to salvage treatment but regrettably four patients experienced a further unresectable local recurrence that could only be treated palliatively. Two of the 28 patients with a local regrowth developed late metastases, after 2 and 4, and it cannot be ruled out that the regrowth was the source of the metastases. Smith et al. reported a retrospective series of on watch-and-wait in 32 patients with a median follow-up of 28 months with six patients developing a local regrowth (19\%). All six regrowths could be salvaged by standard TME and remained locally controlled. Two of the six patients developed late metastatic disease about a year later and again it cannot be ruled out that the regrowth was the source of the late metastases ${ }^{5}$. In the prospective trail of Appelt et al. with a median follow-up of 24 months, 9/40 patients who entered the watch and wait programme developed a local regrowth (23\%). All regrowths were detected within 2 years of follow-up and underwent salvage surgery. One patient with a regrowth developed distant metastases before the local regrowth ${ }^{6}$.

The series of Habr-Gama et al. ${ }^{12}$ illustrates that a watch-and-wait policy carries a small but real oncological risk. That this risk seems lower in the present series may be due to chance, a shorter follow-up, or can be related to a more strict selection and follow-up program that includes state-of-the-art MRI techniques. Only an estimated 17\% of the patients treated primarily in our centre with chemoradiation were considered cCR, compared to $49 \%$ in the Habr-Gama series. This suggests inclusion of larger tumours in our series and possibly also a more strict selection in our series. In the first phase of the present study ${ }^{4}$ we adhered strictly to the predefined selection criteria of a completely normalized rectum on DRE, endoscopy and MRI. There was only one local regrowth in 21 patients, but one third of complete responders were missed and underwent TME. Following this observation it was decided to include TEM or a second assessment after 3 months for patients now considered as 'near complete responders' (Table 1). Although patients that were included at a second assessment after 3 months were more likely to develop a local regrowth (3yr LR 23\% vs 12\%) we did not observe a compromised outcome with regard to DMFS and OS. In the absence of randomized data and very large series it is difficult to calculate an exact oncological risk but based on our study and the two series described above we estimate the excess oncological risk of dying with a watch-and-wait policy in the order of 2-3\% at most. In the shared decision process with the patient this needs to be carefully balanced against the operative risk and loss of function of major rectal surgery. We experienced that patients are often willing to take this small potential risk in order to avoid major surgery, potentially poor functional outcome, or a colostomy. Despite the limitations of decision-analytic modelling it is reassuring that a recent study showed a calculated operative risk in elderly and comorbid patients that was higher than the oncological risk, and in younger patients the risk was equal ${ }^{13}$. 
An alternative to repeated assessment in patients with a 'near complete response' is a local excision, preferably TEM, providing histological proof of a ypT0. The risk of residual lymph node metastasis in ypT0 is 5\%, as shown in a meta-analysis ${ }^{1}$. In the current study a ypT1-2 tumour that was completely resected by TEM and without evidence of lymph node metastases on MRI was considered a candidate for organ-preservation. However, three of five patients with ypT2 residual tumour developed a local regrowth, conforming earlier reports of high recurrence rates for ypT2, and we now strongly recommend immediate completion surgery ${ }^{14-16}$. There are some disadvantages of a TEM. We noted a more difficult follow-up due to fibrotic changes, and salvage TME can also be more difficult ${ }^{14,17}$. Additionally in our series three out of seven patients had major incontinence after TEM at the functional evaluation, compared to only one of $20 \mathrm{~W} \& \mathrm{~W}$ patients. It is unclear if this is related to the higher risk of post-operative complications as compared to TEM without CRT9, 14, 18-20. Although it is likely that TEM can prevent a number of local regrowths, we feel that its role in organ-preservation of rectal cancer is not yet clearly defined.

There are some limitations to this study. First, a median follow-up of 38 months is generally considered short for good estimates for DMSF and OS. Since most regrowths in both our series and other series have occurred within the first 18-24 months and 52\% patients in our series had a follow-up of $>3$ years, we believe that the results will not change much with a longer follow up. Second, the number of patients is relatively small for analyses of differences between subgroups.

\section{Future perspectives}

In the present study, the majority of patients received chemoradiation with the goal to improve local control after TME surgery for more advanced and distal tumours, not with a deliberate goal to preserve the rectum. Patients were offered a watch-and-wait policy only when they appeared to have a CCR at restaging. From the patients diagnosed and treated in our own catchment area the rate of CCR in these tumours is estimated at 17\%. Smaller tumours have a higher chance for a complete response, and a number of trials on (chemo) radiation with the explicit goal of rectal preservation in relatively small tumours have shown that this can be achieved in over $50 \%$ of patients ${ }^{18,21}$. The downside of this approach is that the patients who still need major surgery may end up with a worse function than if they would have had TME surgery without neoadjuvant therapy. Better prediction methods for response to radiotherapy at time of initial diagnosis would better allow a treatment choice that minimizes toxicity of futile neoadjuvant radiotherapy. 


\section{Conclusion}

Organ-preserving treatment appears to be an oncological safe option for selected rectal cancer patients with a clinical complete response after neoadjuvant chemoradiation. When a near complete response is observed, a reassessment after 3 months or a TEM are alternative options to radical surgery, enabling organ-preservation in more patients.

\section{References}

1. Maas M, Nelemans PJ, Valentini V, et al. Long-term outcome in patients with a pathological complete response after chemoradiation for rectal cancer: a pooled analysis of individual patient data. The lancet oncology 2010; 11(9): 835-44.

2. Habr-Gama A, Perez RO, Nadalin W, et al. Operative versus nonoperative treatment for stage 0 distal rectal cancer following chemoradiation therapy: long-term results. Annals of surgery 2004; 240(4): 711-7; discussion 7-8.

3. Ayloor Seshadri R, Kondaveeti SS, Jayanand SB, et al. Complete clinical response to neoadjuvant chemoradiation in rectal cancers: can surgery be avoided? Hepatogastroenterology 2013; 60(123): 410-4.

4. Maas M, Beets-Tan RG, Lambregts DM, et al. Wait-and-see policy for clinical complete responders after chemoradiation for rectal cancer. J Clin Oncol 2011; 29(35): 4633-40.

5. Smith JD, Ruby JA, Goodman KA, et al. Nonoperative management of rectal cancer with complete clinical response after neoadjuvant therapy. Annals of surgery 2012; 256(6): 965-72.

6. Appelt AL, Ploen J, Harling H, et al. High-dose chemoradiotherapy and watchful waiting for distal rectal cancer: a prospective observational study. The lancet oncology 2015; 16(8): 919-27.

7. Coco C, Rizzo G, Mattana C, et al. Transanal endoscopic microsurgery after neoadjuvant radiochemotherapy for locally advanced extraperitoneal rectal cancer: short-term morbidity and functional outcome. Surgical endoscopy 2013; 27(8): 2860-7.

8. Lezoche G, Baldarelli M, Guerrieri M, et al. A prospective randomized study with a 5-year minimum follow-up evaluation of transanal endoscopic microsurgery versus laparoscopic total mesorectal excision after neoadjuvant therapy. Surgical endoscopy 2008; 22(2): 352-8.

9. Perez RO, Habr-Gama A, Sao Juliao GP, Proscurshim I, Scanavini Neto A, Gama-Rodrigues J. Transanal endoscopic microsurgery for residual rectal cancer after neoadjuvant chemoradiation therapy is associated with significant immediate pain and hospital readmission rates. Dis Colon Rectum 2011; 54(5): 545-51.

10. Dindo D, Demartines N, Clavien PA. Classification of surgical complications: a new proposal with evaluation in a cohort of 6336 patients and results of a survey. Annals of surgery 2004; 240(2): 20513.

11. Vaizey CJ, Carapeti E, Cahill JA, Kamm MA. Prospective comparison of faecal incontinence grading systems. Gut 1999; 44(1): 77-80.

12. Habr-Gama A, Gama-Rodrigues J, Sao Juliao GP, et al. Local recurrence after complete clinical response and watch and wait in rectal cancer after neoadjuvant chemoradiation: impact of salvage therapy on local disease control. International journal of radiation oncology, biology, physics 2014; 88(4): 822-8. 
13. Smith FM, Rao C, Oliva Perez R, et al. Avoiding radical surgery improves early survival in elderly patients with rectal cancer, demonstrating complete clinical response after neoadjuvant therapy: results of a decision-analytic model. Dis Colon Rectum 2015; 58(2): 159-71.

14. Bujko K, Richter P, Smith FM, et al. Preoperative radiotherapy and local excision of rectal cancer with immediate radical re-operation for poor responders: a prospective multicentre study. Radiother Oncol 2013; 106(2): 198-205.

15. Stipa F, Picchio M, Burza A, Soricelli E, Vitelli CE. Long-term outcome of local excision after preoperative chemoradiation for ypT0 rectal cancer. Dis Colon Rectum 2014; 57(11): 1245-52.

16. Perez RO, Habr-Gama A, Lynn PB, et al. Transanal endoscopic microsurgery for residual rectal cancer (ypT0-2) following neoadjuvant chemoradiation therapy: another word of caution. Dis Colon Rectum 2013; 56(1): 6-13.

17. Morino M, Allaix ME, Arolfo S, Arezzo A. Previous transanal endoscopic microsurgery for rectal cancer represents a risk factor for an increased abdominoperineal resection rate. Surgical endoscopy 2013; 27(9): 3315-21.

18. Garcia-Aguilar J, Shi Q, Thomas CR, Jr., et al. A phase II trial of neoadjuvant chemoradiation and local excision for T2NO rectal cancer: preliminary results of the ACOSOG Z6041 trial. Ann Surg Oncol 2012; 19(2): 384-91.

19. Gornicki A, Richter P, Polkowski W, et al. Anorectal and sexual functions after preoperative radiotherapy and full-thickness local excision of rectal cancer. European journal of surgical oncology: the journal of the European Society of Surgical Oncology and the British Association of Surgical Oncology 2014; 40(6): 723-30.

20. Marks J, Nassif G, Schoonyoung H, et al. Sphincter-sparing surgery for adenocarcinoma of the distal $3 \mathrm{~cm}$ of the true rectum: results after neoadjuvant therapy and minimally invasive radical surgery or local excision. Surgical endoscopy 2013; 27(12): 4469-77.

21. Verseveld M, de Graaf EJ, Verhoef C, et al. Chemoradiation therapy for rectal cancer in the distal rectum followed by organ-sparing transanal endoscopic microsurgery (CARTS study). Br J Surg 2015; 102(7): 853-60. 


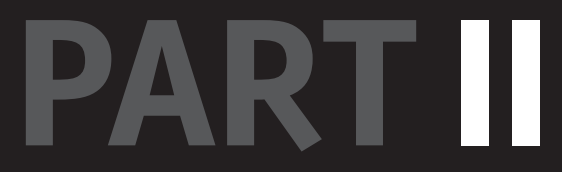

Response assessment after therapy 


\section{CHAPTER 3}

Prospective multicenter validation study of MRI volumetry for response assessment after preoperative chemoradiation in rectal cancer: can the results in literature be reproduced?

Authors:

M.H. Martens, M.M. van Heeswijk, J.J. van den Broek,

S-X. Rao,

V. Vandecaveye,

R.A. Vliegen,

W.H. Schreurs,

G.L. Beets,

D.M.J. Lambregts,

R.G.H. Beets-Tan

International Journal of Radiation Oncology, Biology, Physics, 2015 Sept 15. In press accepted manuscript 


\section{Abstract}

\section{Purpose}

To review available literature on tumor size/volume measurements on MRI for response assessment after chemoradiotherapy, and validate these cut-offs in an independent multicenter patient cohort.

\section{Methods}

The study included two parts:

[1] Review of literature; papers were included that assessed the accuracy of tumor size/ volume measurements on MRI for tumor response assessment. Size/volume cut-offs were extracted.

[2] Multicenter validation; Extracted cut-offs from literature were tested in a multicenter cohort ( $n=146$ ). Accuracies were calculated and compared to reported results from literature.

\section{Results}

The review included fourteen papers, in which 3 different measurement methods were assessed: [1] tumor length, [2] 3-dimensonial tumor size, and [3] whole volume. Study outcomes consisted of [1] complete response (ypT0) versus residual tumor, [2] tumor regression grade (TRG) 1-2 versus 3-5, and [3] T-downstaging (ypT<cT).

In the multicenter cohort, best results were obtained for the validation of the whole-volume measurements, in particular for the outcome ypT0 (accuracy 44-80\%), with the optimal cutoffs being $1.6 \mathrm{~cm}^{3}$ (post-CRT) and a volume-reduction of $\triangle 80-86.6 \%$. Accuracies for wholevolume measurements to assess TRG1-2 were 52-61\%, and for T-downstaging 51-57\%. Overall accuracies for tumor length ranged between 48-53\% and for 3D-size measurement between $52-56 \%$.

\section{Conclusions}

MR-volumetry using whole tumor volume measurements can be helpful in rectal cancer response assessment with selected cut-off values. Measurements of tumor length or threedimensional tumor size are not helpful. MRI volumetry is mainly accurate to assess a complete tumor response (ypT0) after CRT (accuracies up to 80\%). 


\section{Introduction}

Since the introduction of standardized total mesorectal excision (TME) and preoperative chemoradiation treatment (CRT), the outcome for rectal cancer patients has considerably improved ${ }^{1-3}$. Preoperative treatment often results in downsizing and downstaging of the tumor $^{3,4}$, and in $15-20 \%$ of the patients even in a complete tumor regression ${ }^{5}$. In patients with a clinically complete or near complete response, organ-preservation (local excision or "watch-and-wait") is now increasingly considered as a potentially good alternative to surgical resection ${ }^{6-8}$. The recommended tools to assess tumor response include digital rectal examination, endoscopy and imaging (mainly MRI). MRI, approximately 6-8 weeks after completion of chemoradiotherapy ${ }^{9,10}$, performs well in assessing tumor regression from initially invaded structures and can be beneficial in determining the definitive surgical approach ${ }^{11}$. Although MRI has proven useful to assess changes in tumor volume and morphology ${ }^{12}$, evaluation of minimal residual tumor within post-radiation fibrosis remains difficult. As a result, the performance of MRI to assess response after chemoradiotherapy remains limited, as was shown in a recent meta-analysis with an overall sensitivity of $50.4 \%$ and specificity of $91.2 \%$ for MRI in restaging tumor after $\mathrm{CRT}^{10}$. Various studies have investigated the value of measuring changes in the tumor size and/or volume on MRI as a predictor of response. Reported results for these measurements vary considerably, with overall accuracies ranging between $42 \%-88 \% \%^{13-27}$. This could partly be explained by the variety in methods for the tumor size/volume measurements (e.g. one-dimensional versus 3-dimensional versus 'whole-volume' volumetry), by the variety in cut-off values, by the retrospective nature of the majority of often small-scale studies, and the different study endpoints used to define response. Furthermore, prospective validation of retrospectively determined cut-offs in an independent and large multicenter patient cohort is lacking.

Aim of the current study was threefold: [1] to systematically review the available literature on the value of tumor size/volume measurements on MRI (using routine T2-weighted sequences) for tumor response prediction and assessment in patients with rectal cancer undergoing preoperative CRT, [2] to prospectively validate the cut-off values reported in literature in an independent multicenter patient cohort, and [3] to evaluate - based on the above two steps - whether MRI volumetry is reliable to predict and assess primary tumor response and which method and cut-off values would be most accurate.

\section{Materials and Methods}

The study consisted of two parts: [1] systematic review of the available literature and [2] multicenter validation of the data derived from the literature review. 


\section{Literature review}

A literature search was performed to identify all papers focusing on assessment of response to neoadjuvant treatment in patients with locally advanced rectal cancer by means of tumor size or volumetric measurements using routine morphological (T2-weighted) MRI. We performed a search of three major databases: Embase, Medline, and Cochrane library using (a combination of) the following key words: "rectal cancer", "rectal adenocarcinoma", "rectal neoplasm", "response evaluation”, "response assessment”, "response prediction", "radiotherapy", "neoadjuvant therapy", chemoradiation”, "chemoradiotherapy", "tumor length", "tumor volumetry", "tumor size", "tumor volume”, "tumor burden”, "tumor regression grade", "tumor regression", "downstaging", and "pathological complete response". Inclusion criteria were: (1) tumor size/length or volume was measured on T2-weighted MRI before and/ or after neoadjuvant therapy, (2) the main study outcome was the response to neoadjuvant treatment with the standard of reference being pathological complete response, tumordownstaging (cT > pT), or favorable tumor regression grade (TRG), (3) cut-off values for tumor size/volume were used to determine the diagnostic performance for predicting response, and (4) the results were reported in $2 \times 2$ contingency tables or these could otherwise be derived from the data reported. Two reviewers checked the titles and abstracts of the identified studies to determine studies which potentially met the inclusion criteria. Additional studies were identified by checking the reference list of the selected studies. Full text copies were then studied to decide which articles met the final inclusion criteria.

\section{Validation study}

\section{Patients}

According to our country's national law, institutional review board approval is not required for this retrospective study and patients consent was waived. For the validation study, 146 locally advanced rectal cancer patients were included between 2006-2013 in one of four study centers ( 2 academic referral centers and 2 non-academic centers). None of the patients were previously included in any of the previously reported papers included in the review. Inclusion criteria consisted of (1) histologically proven rectal cancer, (2) locally advanced rectal cancer (cT3-4 and/ or $\mathrm{cN}+$ ), (3) long course of neoadjuvant treatment followed by resection, and (4) availability of T2-weighted MRI before and after neoadjuvant treatment. The routine neoadjuvant treatment protocol consisted of either 50.4 Gy radiation combined with $2 \times 825 \mathrm{mg} / \mathrm{m}^{2} /$ day capecitabine or 45 Gy radiation combined with $225 \mathrm{mg} / \mathrm{m}^{2} /$ day 5 -fluorouracil (5-FU). Nineteen patients received an alternative scheme with additional oxaliplatin and/or bevacizumab.

\section{Magnetic Resonance Imaging}

MRI was performed as part of the routine diagnostic workup for both primary staging and restaging after CRT in all centers. The standard interval between completion of CRT and the restaging MRI was 4-8 weeks in all centers. The standard MRI protocol included T2-weighted Fast Spin Echo (FSE) sequences in 3 orthogonal directions. Slice thickness was 3-3.5 mm. 
Detailed information on scanning systems, patient preparation, and sequence parameters are described in Table 1.

Table 1. Overview of hardware, sequence parameters and patient preparation.

\begin{tabular}{|l|l|l|l|l|l|}
\hline Parameter & Center 1 & Center 2 & Center 3 & $\begin{array}{l}\text { Center 4 } \\
\text { Protocol 1 }\end{array}$ & $\begin{array}{l}\text { Center 4 } \\
\text { Protocol 2 }\end{array}$ \\
\hline Manufacturer & Philips & Siemens & Siemens & Siemens & Philips \\
\hline Field Strength (T) & 1.5 & 1.5 & 1.5 & 1.5 & 3.0 \\
\hline Type of coil & Surface coil & Surface coil & Surface coil & Surface coil & Surface coil \\
\hline Repetition time (ms) & $8456-11788$ & 4000 & $3400-4160$ & 8870 & 5000 \\
\hline Echo time (ms) & 130 & 102 & 122 & 134 & 95 \\
\hline No. Slices & 30 & 32 & 48 & 20 & 32 \\
\hline Slice thickness (mm) & 3 & 3.5 & 3.5 & 3.5 & 3 \\
\hline Slice gap (mm) & 2 & 0.7 & $0-0.35$ & 0.7 & 0 \\
\hline $\begin{array}{l}\text { In plane resolution } \mathbf{m m} \text { x } \\
\text { mm) }\end{array}$ & $0.78^{\star} 1.14$ & $0.7 * 0.6$ & $1.0^{*} 0.7$ & $0.63^{\star} 0.73$ & $0.69^{\star} 0.82$ \\
\hline Echo train length & 25 & 23 & 23 & 61 & 19 \\
\hline No. signal averages & 2 & 3 & 2 & 3 & 1 \\
\hline Bowel preparation & No & No & No & $\begin{array}{l}\text { Endorectal } \\
\text { filling }\end{array}$ & $\begin{array}{l}\text { Endorectal } \\
\text { filling }\end{array}$ \\
\hline
\end{tabular}

\section{Image evaluation}

MR images were evaluated on an offline workstation using the closed polygon tool in Osirix Medical Imaging Software ${ }^{28}$ by one reader with 8 years of specific rectal MRI experience (S$X R)$. A second reader with 3 years of experience (MHM) re-evaluated the pre- and post-CRT images of a random sample of one third $(n=48)$ of the study patients to evaluate interobserver reproducibility. The readers were blinded to each other's results and all clinical and histopathological data. Tumor size and volume measurements (including only the tumor in the rectal wall, excluding any lymph nodes or extraluminal tumor deposits) were performed on the primary staging MRI as well as on the restaging MRI. Measurements were manually performed according to 3 different methods (concordant with different methodologies described in literature): [1] One-dimensional size measurement of the maximum tumor length on sagittal MRI 15,27, [2] 3-dimensional size measurements (i.e., tumor length (sagittal plane) x anterior-posterior (AP) diameter (axial plane) x left-right (LR) diameter (axial plane)) ${ }^{26,27}$, and [3] measurement of the whole tumor volume by manually tracing the tumor boundaries on each tumor-containing slice and multiplying these cross-sectional areas by the slice thickness (including the slice gap) ${ }^{13-25}$. For all 3 methods the percentage of volume/size reduction $\left(V_{\Delta}\right)$ was calculated by $\left(V_{\text {pre }}-V_{\text {post }}\right) V_{\text {pre }} \times 100$, with $V_{\text {pre }}$ and $V_{\text {post }}$ representing the volumes/sizes preand post-CRT, respectively. The different measurement methods are illustrated in Figure 1. 

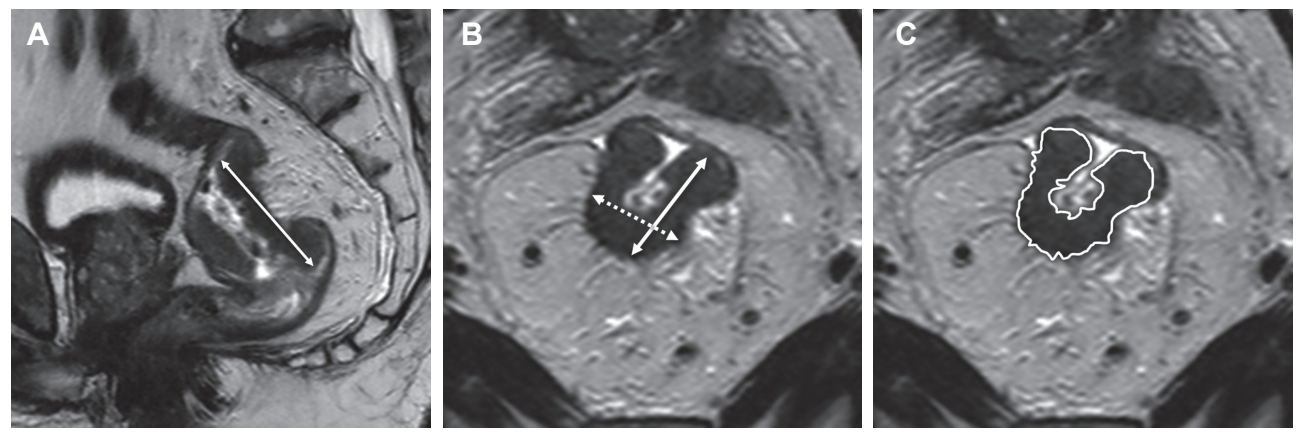

\section{Figure 1.}

(A) T2-weighted sagittal MRI, the arrow indicates the maximal tumor length (method 1). (B) T2-weighted axial MRI, the dashed arrow indicates the left-right (LR) diameter, the filled arrow represents the anterior-posterior (AP) diameter (method 2: length $x$ LR $\times$ AP). (C) Delineation of the tumor boundaries for method 3 (tumor area $x$ slice thickness).

\section{Reference standard}

All patients underwent surgical resection. Histopathological examination was performed according to the method described by Quirke et $a^{22}$. The pathological T-stage (ypT) was reported for each patient. Tumor regression grade was assessed as part of routine clinical care according to the method described by Mandard ${ }^{30}$ or Dworak ${ }^{31}$. For study purposes, the TRGs measured according to the Dworak classification were converted to the method of Mandard, with TRG 1-2 (concordant with Dworak TRG 3-4) being a good response and TRG 3-5 (concordant with Dworak TRG 0-2) being a poor response ${ }^{32}$.

\section{Statistical Analyses}

Statistical analyses were performed using the Statistical Package for the Social Sciences (SPSS, version 20.0, Inc., Chicago, Illinois, USA). For the measurements performed by both readers $(n=48)$, interobserver agreement $(I O A)$ was evaluated by calculating the intraclass correlation coefficient (ICC) with ICC 0-0.20 indicating poor, 0.21-0.40 fair, 0.41-0.60 moderate, 0.61-0.80 good and 0.81-1.0 indicating excellent agreement. For all other analyses, the measurements of reader 1 were used. Non-parametric Mann-Whitney $U$ test was performed to compare the volumes between good/poor responders. Dichotomization between good/ poor responders was performed according to different methods derived from the literature review: [1] complete response (ypT0) vs residual tumor, [2] Tumor-downstaging (cT-stage > ypT-stage) vs no Tumor-downstaging (cT-stage $\leq$ ypT-stage), and [3] TRG 1-2 vs TRG3-5, where a favorable response (i.e. ypT0, cT>pT, TRG1-2) was consistently defined as the positive outcome. Lymph nodes were not taken into account. P-values $<0.05$ were considered statistically significant. Sensitivities, specificities, positive predictive values (PPV), negative predictive values (NPV), and overall accuracies including 95\% confidence intervals (CI) for identifying a good response were calculated using the predefined cut-off values derived from the results of the literature review. 


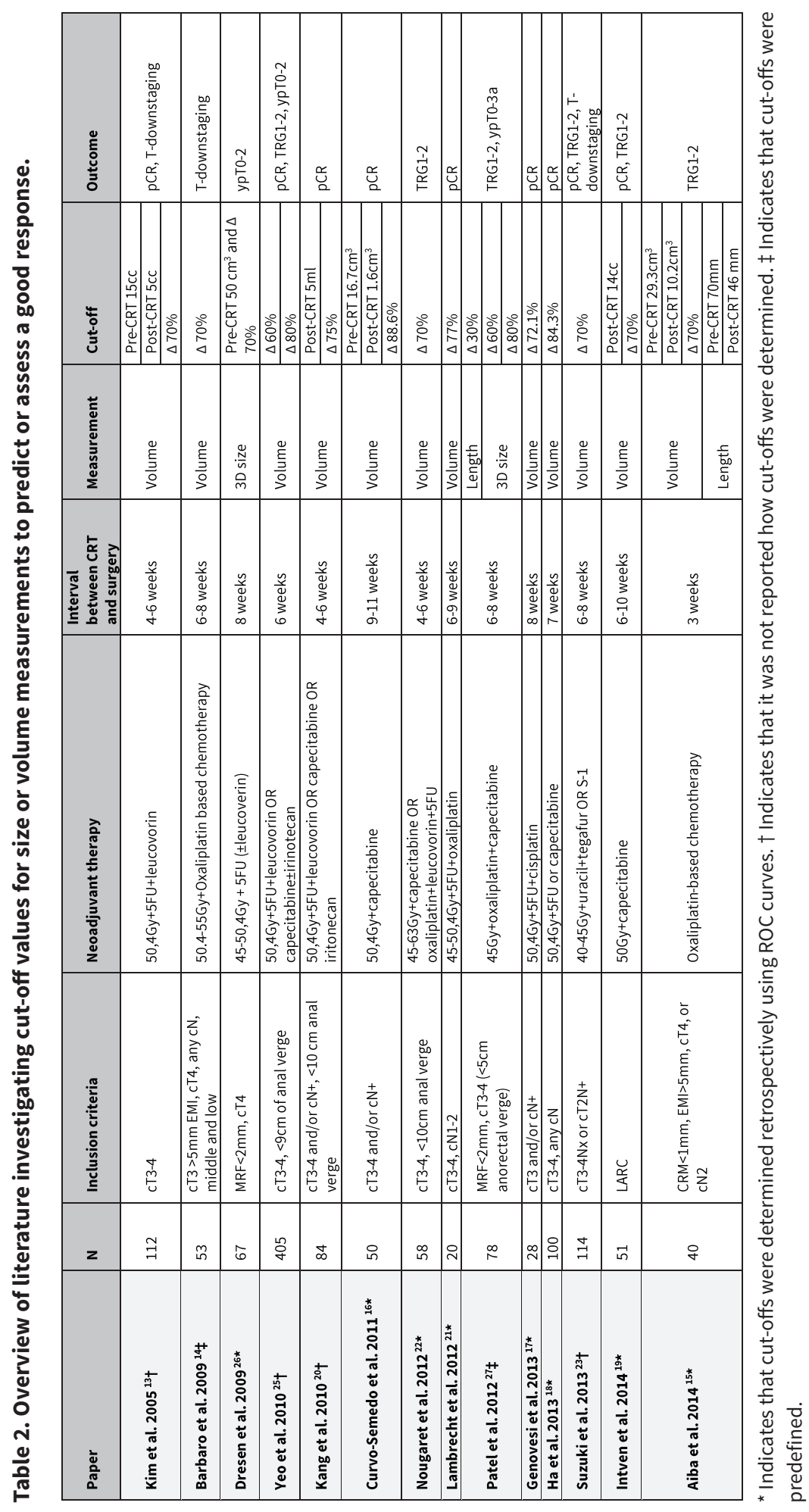




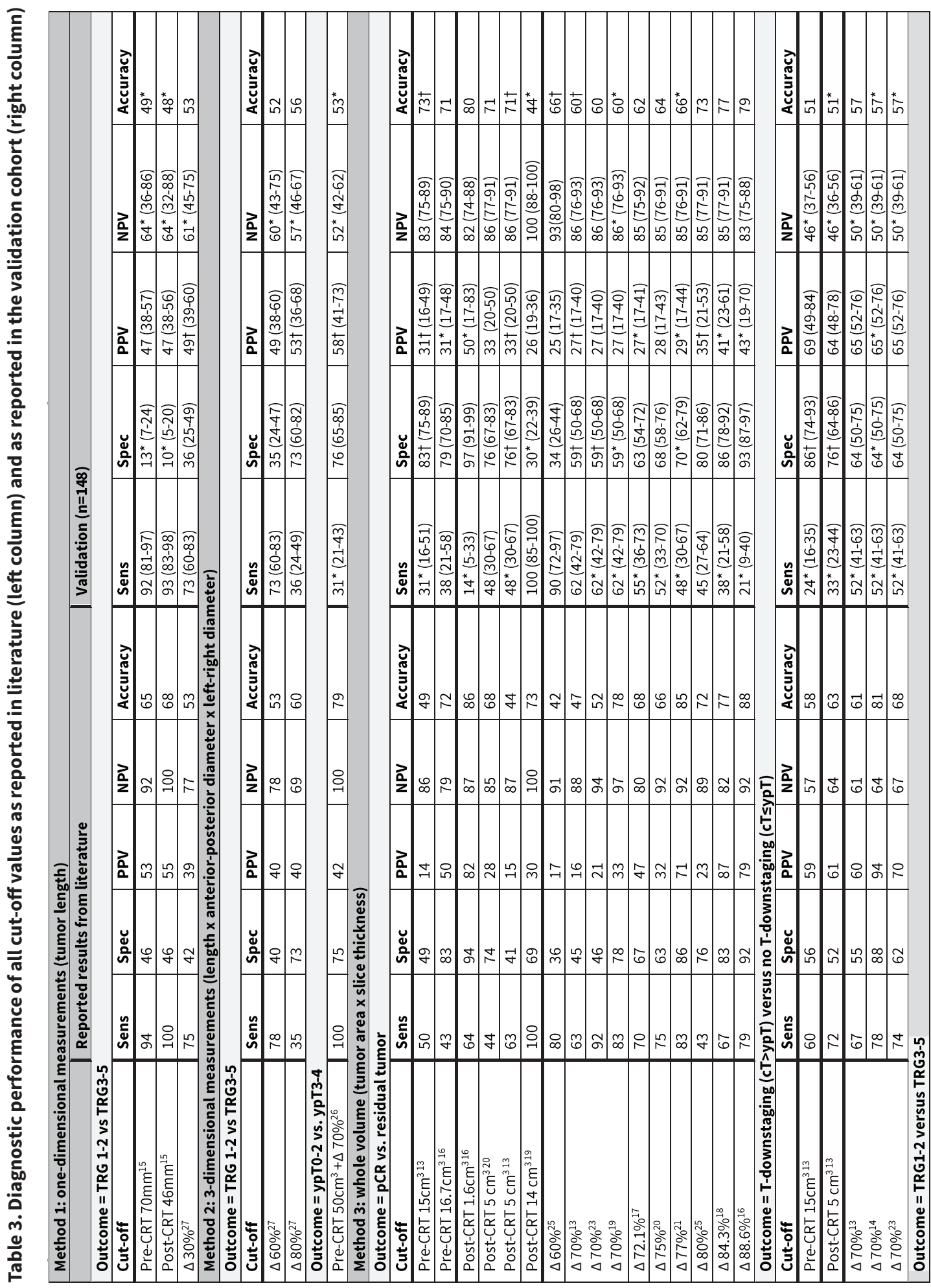



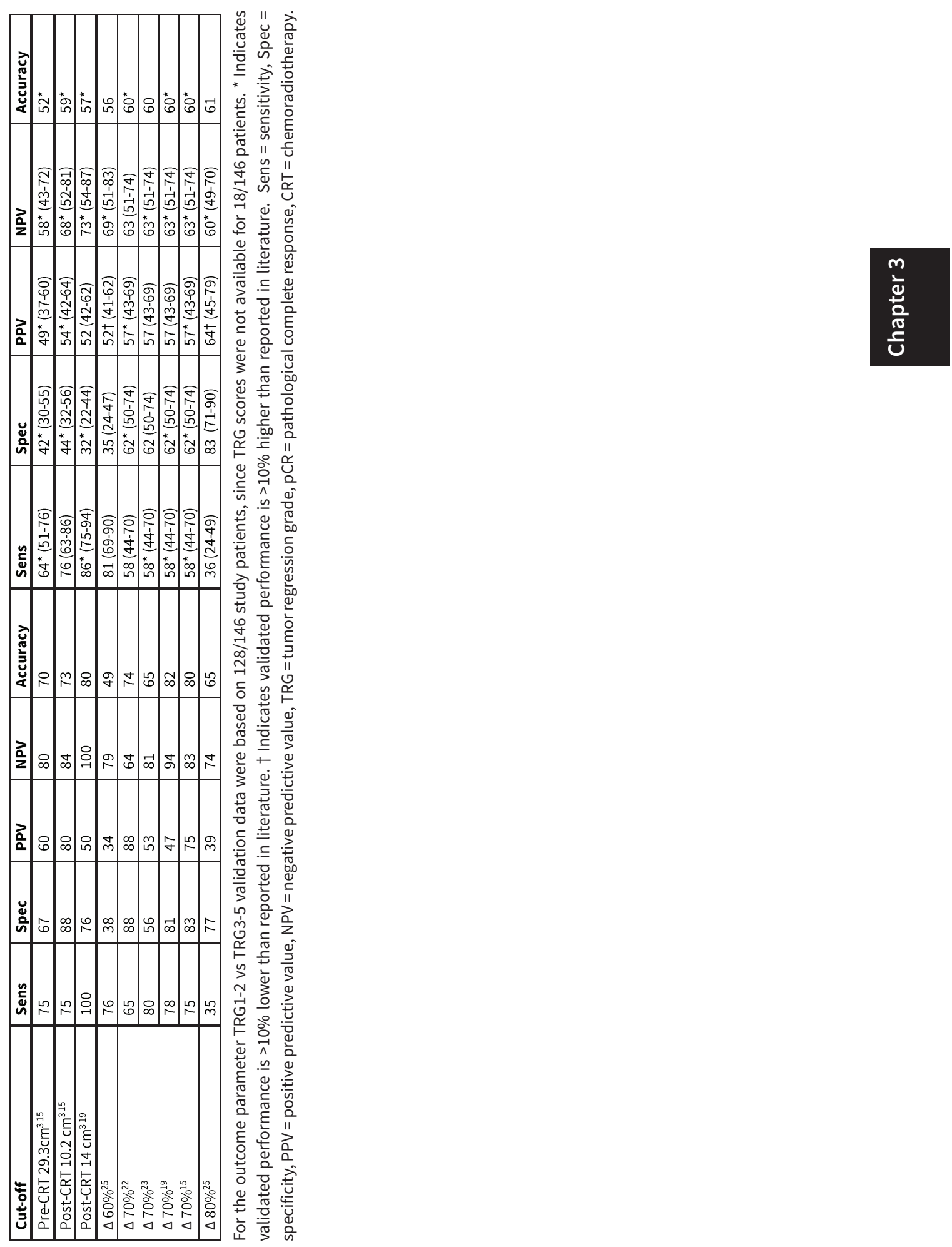


\section{Results}

\section{Literature Review}

Fourteen papers met the final inclusion criteria. An overview of these studies is presented in Table 2. Two papers focused on the one-dimensional tumor size (method 1$)^{15,27}$. Two papers evaluated the 3-dimensional tumor size (method 2) ${ }^{26,27}$. The majority of the papers $(12 / 14)$ focused on whole tumor volume measurements (method 3). In 2 of the 14 studies, cut-off values used were predefined before onset of the study ${ }^{14,27}$. In the other 12 studies, cut-off values were either retrospectively determined from the study dataset ${ }^{15-19,21,22,26}$ or it was not clearly reported how these were determined ${ }^{13,20,23,25}$. The results (i.e. accuracy figures) of the different studies are given in Table 3.

\section{Prospective validation study}

\section{Patient and treatment characteristics}

Of the 146 study patients 90 were male and 56 were female (mean age 64.6 years, \pm 10.8 ). Mean interval between the completion of radiation and restaging MRI was 5.7 weeks $( \pm 2.4)$, and mean interval between the completion of radiation and surgery was 10 weeks $( \pm 3.8)$. Further patient and treatment details are given in Table 4.

\section{Interobserver agreement}

Interobserver agreement for the one-dimensional measurements (method 1) was good preCRT (ICC 0.78) and post-CRT (ICC 0.66). For the 3-dimensional measurements (method 2) agreement was moderate pre-CRT (ICC 0.60) and good post-CRT (ICC 0.61). For the wholevolume measurements (method 3), agreement was excellent pre-CRT (ICC 0.94) and good post-CRT (ICC 0.65).

\section{Tumor size and volume measurements.}

Mean tumor length (method 1 ) was $5.1 \mathrm{~cm}( \pm 1.5 \mathrm{~cm})$ before therapy and $3.1 \mathrm{~cm}( \pm 1.1 \mathrm{~cm})$ posttherapy, with a mean decrease $(\Delta)$ of $-36.6 \%$ ( $\pm 20.2 \%)$. Mean volumes according to method 2 (length $\times$ AP $\times$ LR) were $57.9 \mathrm{~cm}^{3}$ pre-CRT $\left( \pm 50.6 \mathrm{~cm}^{3}\right), 17.0 \mathrm{~cm}^{3}$ post-CRT $\left( \pm 16.1 \mathrm{~cm}^{3}\right)$, and $V_{\Delta}$ was $-65.8 \%( \pm 23.9 \%)$. According to the whole volume measurements (method 3), mean volumes were $32.8 \mathrm{~cm}^{3}$ pre-CRT $\left( \pm 25.6 \mathrm{~cm}^{3}\right), 10.9 \mathrm{~cm}^{3}$ post-CRT $\left( \pm 11.3 \mathrm{~cm}^{3}\right)$, and $V_{\Delta}$ was $64.6 \%( \pm 22.4 \%)$.

\section{Diagnostic performance of predefined cut-off values and comparison with literature}

Diagnostic accuracy figures using the cut-off values derived from the literature review are provided in Table 3, together with those originally reported in literature.

[1] For the one-dimensional size measurements overall accuracy (vs. that originally reported in literature) to predict TRG1-2 ranged from 48-53\% (vs. 53-68\%), sensitivity was 73-93\% (vs. 
75-100\%) and specificity 10-36\% (vs. 42-46\%); the outcomes ypT0 and T-downstaging were not reported/studied.

[2] For the 3-dimensional size measurements overall accuracy to predict TRG 1-2 was 52-56\% (vs. 53-60\%), sensitivity 36-73\% (vs. 35-78\%) and specificity 35-73\% (vs. 40-73\% ); to predict ypT0-2 accuracy was 53\% (vs. 79\%), sensitivity 31\% (vs. 100\%) and specificity 76\% (vs. 75\%); the outcome ypT0 was not reported/studied.

[3] For the whole-volume measurements accuracy to predict a complete response (ypT0) was $44-80 \%$ (vs. 42-88\%), sensitivity $14-100 \%$ (vs. 43-100\%) and specificity 30-97\% (vs. 3694\%); to predict T-downstaging accuracy was 51-57\% (vs. 58-61\%), sensitivity $24-52 \%$ (vs. 60-78\%) and specificity 64-86\% (vs. 52-88\%); to predict TRG1-2 accuracy was 52-61\% (vs. 4982\%), sensitivity $36-86 \%$ (vs. 35-100\%) and specificity 32-83\% (vs. 38-88\%).

When comparing the results for the multicenter validation with those originally reported in literature, the prospectively calculated accuracy was $>10 \%$ lower than originally reported in literature in $43 \%$ of the tested cut-offs, in $11 \%$ overall accuracy was $>10 \%$ higher than originally reported and in the other $46 \%$ accuracy was similar (within a 10\% range) to the originally reported data.

\section{Discussion}

Aim of this study was threefold. The first aim was to systematically review all available literature investigating the value of tumor size/volume measurements on MRI to predict and assess tumor response to CRT in patients with locally advanced rectal cancer. The second aim was to prospectively validate different cut-off values derived from the literature review in an independent multicenter cohort of rectal cancer patients treated with CRT. The third aim was to evaluate - based on the former - whether MRI volumetry is reliable to predict and assess the response of the primary tumor to CRT and which method and cut-off values would be most accurate.

As expected, the diagnostic performance of retrospectively determined cut-off values from literature decreased when tested 'prospectively' in an independent new cohort of patients. Of the fourteen papers included in the review, cut-off values had been determined retrospectively from the study data in the majority (8/14) of studies, while another four papers did not report how cut-off values had been defined. Only two papers had defined the cut-off values beforehand and already applied the cut-offs prospectively in their study. For $43 \%$ of the cut-offs that were tested, the overall accuracies in our prospective multicenter validation cohort were at least 10\% lower compared to the results reported in the original studies. Similarly, the sensitivity and NPV were at least 10\% lower than initially reported in $44 \%$ and 56\%, respectively. The specificity and PPV on the other hand were typically similar or even $>10 \%$ better than initially reported. As one would expect, a certain tradeoff between sensitivity and specificity was observed depending on the chosen cut-off values. A stricter cut-off will result in a higher specificity at the expense of a lower sensitivity and vice versa. 
Table 4. Patient and treatment characteristics

\begin{tabular}{|c|c|c|}
\hline Parameter & Categories & Total $(n=146)$ \\
\hline Age $[\operatorname{mean}( \pm S D)]$ & Years & $64.6( \pm 10.8)$ \\
\hline $\operatorname{Sex}[n(\%)]$ & $\begin{array}{l}\text { Male } \\
\text { female }\end{array}$ & $\begin{array}{l}90(61.6) \\
56(38.4)\end{array}$ \\
\hline Clinical T-stage [n (\%)] & $\begin{array}{l}\text { CT2 } \\
\text { CT3 } \\
\text { CT4 }\end{array}$ & $\begin{array}{l}2(1.4) \\
122(83.6) \\
22(15.1)\end{array}$ \\
\hline MRF involvement [n (\%)] & $\begin{array}{l}\text { No } \\
\text { Threatened }(<2 \mathrm{~mm}) \\
\text { Involvement }\end{array}$ & $\begin{array}{l}61(41.8) \\
38(26) \\
47(32.2)\end{array}$ \\
\hline Distance from anorectal verge [n (\%)] & $\begin{array}{l}0-5 \mathrm{~cm} \\
5-10 \mathrm{~cm} \\
10-15 \mathrm{~cm}\end{array}$ & $\begin{array}{l}78(53.4) \\
61(41.8) \\
7(4.8)\end{array}$ \\
\hline Clinical N-stage [n (\%)] & $\begin{array}{l}\mathrm{CN} 0 \\
\mathrm{CN} 1 \\
\mathrm{CN} 2\end{array}$ & $\begin{array}{l}16(11) \\
34(23.3) \\
96(65.8)\end{array}$ \\
\hline Neoadjuvant treatment [n (\%)] & $\begin{array}{l}45 G y+5 F U \\
50.4 G y+\text { Capecitabine } \\
\text { Other }\end{array}$ & $\begin{array}{l}35(24) \\
92(63.0) \\
19(13.0)\end{array}$ \\
\hline Type of resection [n (\%)] & $\begin{array}{l}\text { LAR } \\
\text { APR } \\
\text { Local excision } \\
\text { TEM } \\
\text { Extended surgery }\end{array}$ & $\begin{array}{l}102(69.9) \\
38(26.2) \\
2(1.4) \\
1(0.7) \\
2(1.4) \\
\end{array}$ \\
\hline Pathogical T-stage [n (\%)] & $\begin{array}{l}\text { урт0 } \\
\text { урT1 } \\
\text { урт2 } \\
\text { урт3 } \\
\text { урT4 }\end{array}$ & $\begin{array}{l}29(19.9) \\
5(3.4) \\
41(28.1) \\
69(47.3) \\
2(1.4)\end{array}$ \\
\hline Pathogical N-stage [n (\%)] & $\begin{array}{l}\text { ypN0 } \\
\text { ypN1 } \\
\text { ypN2 }\end{array}$ & $\begin{array}{l}99(67.8) \\
33(22.6) \\
14(9.6)\end{array}$ \\
\hline $\begin{array}{l}\text { Tumor regression grade according to } \\
\text { Mandard [n (\%)] }\end{array}$ & $\begin{array}{l}1 \\
2 \\
3 \\
4 \\
5 \\
\text { Unknown }\end{array}$ & $\begin{array}{l}30(20.5) \\
29(19.9) \\
47(32.2) \\
18(12.3) \\
4(2.7) \\
18(12.3)\end{array}$ \\
\hline \multirow[t]{3}{*}{ Good vs Poor response [n (\%)] } & $\begin{array}{l}\text { pCR (ypT0) } \\
\text { No pCR }\end{array}$ & $\begin{array}{l}29(19.9) \\
117(80.1)\end{array}$ \\
\hline & $\begin{array}{l}\text { T-downstaging } \\
\text { No T-downstaging }\end{array}$ & $\begin{array}{l}83(56.8) \\
63(43.2)\end{array}$ \\
\hline & $\begin{array}{l}\text { TRG 1-2 } \\
\text { TRG 3-5 } \\
\text { Unknown }\end{array}$ & $\begin{array}{l}59(40.4) \\
69(47.3) \\
18(12.3)\end{array}$ \\
\hline
\end{tabular}

MRF = mesorectal fascia, $5 \mathrm{FU}=5$-fluorouracil, LAR = low anterior resection, $A P R=$ abdominopereanal resection, $\mathrm{TEM}=$ transanal endoscopic microsurgery, $\mathrm{PCR}=$ pathological complete response, $\mathrm{TRG}=$ tumor regression grade. 
An important finding of our study is that both one-dimensional (i.e. tumor length) as well as 3-dimensional size measurements are insufficiently accurate to assess response. Overall accuracy for tumor length measurements in the review ranged between only 53\%-68\% and dropped to $48-53 \%$ in our validation cohort. For the three-dimensional size measurements (length $\times$ AP $\times$ LR), the overall accuracies showed a similar trend; moderate accuracies of $53-79 \%$ were reported in literature dropping to 52-56\% when prospectively re-tested. In contrast, good results were reported for whole-tumor volume with overall accuracies of over $80 \%$ for both post-treatment and $\Delta \%$ measurements, which could be reproduced in our validation cohort, with accuracies up to 79-80\%. Moreover, interobserver agreement was considerably better for the whole-volume measurements (e.g. ICC was 0.94 pre-CRT versus 0.78 for tumor length and 0.60 for three-dimensional size measurements). The wholevolume measurements offered the best results for assessment and prediction of complete response (ypT0) as the outcome variable, with overall accuracies ranging up to $80 \%$. For the other outcome measures - tumor regression grade and T-downstaging - results were considerably poorer with accuracies of only $51-60 \%$. Although we have to take into account that this discrepancy will also partly be influenced by the fact that a complete response is a less prevalent outcome measure than TRG1-2 or T-downstaging, it may also be related to intrinsic differences between the outcome measure ypT0 on the one hand, and TRG and T-downstaging on the other hand. The relation with the reduction of tumor volume is less straightforward in TRG and T-downstaging, than for a complete tumor response. A tumor can show a marked volume reduction but still be ypT3 because of a small remnant in the mesorectum. Likewise a tumor may shrink considerably but still be a non-responder on histological TRG assessment. Additionally, it is known that there is more interobserver variation in assessing the histological TRG, compared to assessing a complete regression or T-downstaging 33 . In our study we specifically focused on the response of the primary tumor itself and did not take into account the evolution in nodal status, which was beyond the scope of this study.

For whole-tumor volumetry, the most promising cut-off values to predict ypT0 - based both on literature and the validation results - were a volume of $1.6 \mathrm{~cm}^{3}$ post-CRT and a volume reduction post-CRT of $\triangle 80-86.6 \%$. The accuracies reported in literature for these cut-offs ranged from $72 \%-88 \%$, prospective validation resulted in comparable overall accuracies ranging from $73 \%-80 \%$, with a corresponding sensitivity of $14-45 \%$ and specificity of $80 \%$ $97 \%$. From a clinical point of view, it is particularly important to achieve a high specificity to ensure that no residual tumors are missed which would induce a risk for recurrence when considering patients for organ-preserving treatments. The high specificity of 80-97\% in our validation cohort indicates that most patients with residual tumor will be correctly identified and patients have a $<3-20 \%$ chance of being falsely classified as complete responders. However, corresponding sensitivities are relatively poor, indicating that many patients with a complete response will be missed and incorrectly diagnosed as having residual tumor. When selecting patients for organ-preservation after CRT using MR volumetry to support clinical 
selection tools would imply a very strict selection, since a substantial number of patients with a complete response might not be identified as such.

In daily radiological practice, a potentially limiting factor of whole-volume tumor volumetry is the fact that it is a time consuming procedure. Future work should therefore focus on the development of post-processing methods such as auto-segmentation techniques that may speed up the process of tumor delineation. In this regard, tumor volumetry using functional imaging techniques such as diffusion-weighted MRI (DWI) or FDG-PET have already been investigated with good sensitivities up to 70\% for assessing a pCR with (semi-)automated segmentation ${ }^{34-36}$. The advantage is that both DWI and FDG-PET have superior strong contrast resolution, a prerequisite for automated tumor delineation.

Our study has some limitations. First, the validation part was performed using routinely acquired MRI data from 4 different hospitals. As such, there were variations in MRI hardware, scanning protocols and patient preparation. This approach however reflects daily clinical practice where protocols are subject to variations between different centers and therefore may render our results more generally applicable. Second, there was a subgroup of patients $(n=19)$ that underwent an alternative neoadjuvant treatment protocol, potentially affecting the treatment response itself. Third, both in literature as well as in our validation cohort, there were variable intervals between imaging and surgery, potentially leading to the underestimation on imaging of the final histopathological response. Fourth, due to the time consuming nature of the study the second reader performed measurements on only a sample of patients to test for interobserver differences. Our interobserver agreement was, however, fully in line with various previously published results on IOA and we therefore believe that our results are representative ${ }^{16,17,22,34}$. Finally, there were some variations in inclusion criteria in the different reports, from which the studied cut-off values were derived. Although overall the selection criteria used to establish our current study group corresponded well with those applied in literature, these slight variations may have affected the study outcome.

In conclusion, we have shown that MRI volumetry using whole tumor volume measurements may be helpful in rectal cancer response assessment when using selected cut-off values. Measurements of tumor length or three-dimensional tumor size are not helpful. MRI volumetry is mainly accurate to assess a complete tumor response (ypT0) after CRT, but less to assess T-downstaging or TRG1-2. Further research should focus on the development of automated segmentation techniques which may accelerate the process of tumor delineation and facilitate its implementation in clinical practice.

\section{References}

1. Heald RJ. A new approach to rectal cancer. British journal of hospital medicine 1979; 22(3):277-81.

2. Kapiteijn E, Marijnen CA, Nagtegaal ID, et al. Preoperative radiotherapy combined with total mesorectal excision for resectable rectal cancer. N Engl J Med 2001; 345(9): 638-46. 
3. Sauer R, Becker H, Hohenberger W, et al. Preoperative versus postoperative chemoradiotherapy for rectal cancer. N Engl J Med 2004; 351(17): 1731-40.

4. Reerink O, Verschueren RC, Szabo BG, Hospers GA, Mulder NH. A favourable pathological stage after neoadjuvant radiochemotherapy in patients with initially irresectable rectal cancer correlates with a favourable prognosis. European journal of cancer 2003; 39(2): 192-5.

5. Maas M, Nelemans PJ, Valentini V, et al. Long-term outcome in patients with a pathological complete response after chemoradiation for rectal cancer: a pooled analysis of individual patient data. The lancet oncology 2010; 11(9): 835-44.

6. Lezoche G, Baldarelli M, Guerrieri M, et al. A prospective randomized study with a 5-year minimum follow-up evaluation of transanal endoscopic microsurgery versus laparoscopic total mesorectal excision after neoadjuvant therapy. Surgical endoscopy 2008; 22(2): 352-8.

7. Maas M, Beets-Tan RG, Lambregts DM, et al. Wait-and-see policy for clinical complete responders after chemoradiation for rectal cancer. J Clin Oncol 2011; 29(35): 4633-40.

8. Habr-Gama A, Perez RO, Nadalin W, et al. Operative versus nonoperative treatment for stage 0 distal rectal cancer following chemoradiation therapy: long-term results. Annals of surgery 2004; 240(4): 711-7; discussion 7-8.

9. Beets-Tan RG, Lambregts DM, Maas M, et al. Magnetic resonance imaging for the clinical management of rectal cancer patients: recommendations from the 2012 European Society of Gastrointestinal and Abdominal Radiology (ESGAR) consensus meeting. Eur Radio/ 2013; 23(9): 2522-31.

10. van der Paardt MP, Zagers MB, Beets-Tan RG, Stoker J, Bipat S. Patients Who Undergo Preoperative Chemoradiotherapy for Locally Advanced Rectal Cancer Restaged by Using Diagnostic MR Imaging: A Systematic Review and Meta-Analysis. Radiology 2013; 269(1): 101-12.

11. Vliegen RF, Beets GL, Lammering $G$, et al. Mesorectal fascia invasion after neoadjuvant chemotherapy and radiation therapy for locally advanced rectal cancer: accuracy of MR imaging for prediction. Radiology 2008; 246(2): 454-62.

12. Barbaro B, Vitale R, Leccisotti L, et al. Restaging locally advanced rectal cancer with MR imaging after chemoradiation therapy. Radiographics: a review publication of the Radiological Society of North America, Inc 2010; 30(3): 699-716.

13. Kim YH, Kim DY, Kim TH, et al. Usefulness of magnetic resonance volumetric evaluation in predicting response to preoperative concurrent chemoradiotherapy in patients with resectable rectal cancer. International journal of radiation oncology, biology, physics 2005; 62(3): 761-8.

14. Barbaro B, Fiorucci C, Tebala C, et al. Locally advanced rectal cancer: MR imaging in prediction of response after preoperative chemotherapy and radiation therapy. Radiology 2009; 250(3): 730-9.

15. Aiba T, Uehara K, Nihashi T, et al. MRI and FDG-PET for assessment of response to neoadjuvant chemotherapy in locally advanced rectal cancer. Ann Surg Oncol 2014; 21(6): 1801-8.

16. Curvo-Semedo L, Lambregts DM, Maas M, et al. Rectal cancer: assessment of complete response to preoperative combined radiation therapy with chemotherapy--conventional MR volumetry versus diffusion-weighted MR imaging. Radiology 2011; 260(3): 734-43.

17. Genovesi D, Filippone A, Ausili Cefaro G, et al. Diffusion-weighted magnetic resonance for prediction of response after neoadjuvant chemoradiation therapy for locally advanced rectal cancer: preliminary results of a monoinstitutional prospective study. European journal of surgical oncology: the journal of the European Society of Surgical Oncology and the British Association of Surgical Oncology 2013; 39(10): 1071-8. 
18. Ha HI, Kim AY, Yu CS, Park SH, Ha HK. Locally advanced rectal cancer: diffusion-weighted MR tumour volumetry and the apparent diffusion coefficient for evaluating complete remission after preoperative chemoradiation therapy. Eur Radio/ 2013; 23(12): 3345-53.

19. Intven M, Reerink O, Philippens ME. Dynamic contrast enhanced MR imaging for rectal cancer response assessment after neo-adjuvant chemoradiation. J Magn Reson Imaging 2015; 41(6): 1646-53.

20. Kang JH, Kim YC, Kim H, et al. Tumor volume changes assessed by three-dimensional magnetic resonance volumetry in rectal cancer patients after preoperative chemoradiation: the impact of the volume reduction ratio on the prediction of pathologic complete response. International journal of radiation oncology, biology, physics 2010; 76(4): 1018-25.

21. Lambrecht M, Vandecaveye V, De Keyzer F, et al. Value of diffusion-weighted magnetic resonance imaging for prediction and early assessment of response to neoadjuvant radiochemotherapy in rectal cancer: preliminary results. International journal of radiation oncology, biology, physics 2012; 82(2): 863-70.

22. Nougaret S, Rouanet P, Molinari N, et al. MR volumetric measurement of low rectal cancer helps predict tumor response and outcome after combined chemotherapy and radiation therapy. Radiology 2012; 263(2): 409-18.

23. Suzuki T, Sadahiro S, Tanaka A, et al. Relationship between histologic response and the degree of tumor shrinkage after chemoradiotherapy in patients with locally advanced rectal cancer. Journal of surgical oncology 2014; 109(7): 659-64.

24. van Stiphout RG, Valentini V, Buijsen J, et al. Nomogram predicting response after chemoradiotherapy in rectal cancer using sequential PETCT imaging: A multicentric prospective study with external validation. Radiother Oncol 2014; 113(2): 215-22.

25. Yeo SG, Kim DY, Kim TH, et al. Tumor volume reduction rate measured by magnetic resonance volumetry correlated with pathologic tumor response of preoperative chemoradiotherapy for rectal cancer. International journal of radiation oncology, biology, physics 2010; 78(1): 164-71.

26. Dresen RC, Beets GL, Rutten HJ, et al. Locally advanced rectal cancer: MR imaging for restaging after neoadjuvant radiation therapy with concomitant chemotherapy. Part I. Are we able to predict tumor confined to the rectal wall? Radiology 2009; 252(1): 71-80.

27. Patel UB, Brown G, Rutten $\mathrm{H}$, et al. Comparison of magnetic resonance imaging and histopathological response to chemoradiotherapy in locally advanced rectal cancer. Ann Surg Oncol 2012; 19(9): 2842-52.

28. Rosset A, Spadola L, Ratib O. OsiriX: an open-source software for navigating in multidimensional DICOM images. J Digit Imaging 2004; 17(3): 205-16.

29. Quirke P, Durdey P, Dixon MF, Williams NS. Local recurrence of rectal adenocarcinoma due to inadequate surgical resection. Histopathological study of lateral tumour spread and surgical excision. Lancet 1986; 2(8514): 996-9.

30. Mandard AM, Dalibard F, Mandard JC, et al. Pathologic assessment of tumor regression after preoperative chemoradiotherapy of esophageal carcinoma. Clinicopathologic correlations. Cancer 1994; 73(11): 2680-6.

31. Dworak O, Keilholz L, Hoffmann A. Pathological features of rectal cancer after preoperative radiochemotherapy. Int J Colorectal Dis 1997; 12(1): 19-23. 
32. Santos MD, Silva C, Rocha A, Matos E, Nogueira C, Lopes C. Prognostic value of mandard and dworak tumor regression grading in rectal cancer: study of a single tertiary center. ISRN surgery 2014; 2014: 310542.

33. Chetty R, Gill P, Govender D, et al. International study group on rectal cancer regression grading: interobserver variability with commonly used regression grading systems. Human pathology 2012; 43(11): 1917-23.

34. Carbone SF, Pirtoli L, Ricci V, et al. Assessment of response to chemoradiation therapy in rectal cancer using MR volumetry based on diffusion-weighted data sets: a preliminary report. $L a$ Radiologia medica 2012; 117(7): 1112-24.

35. Chen SW, Chen WT, Wu YC, et al. Which FDG/PET parameters of the primary tumors in colon or sigmoid cancer provide the best correlation with the pathological findings? European journal of radiology 2013; 82(9): e405-10.

36. Lambregts DM, Rao SX, Sassen S, et al. MRI and Diffusion-Weighted MRI Volumetry for Identification of Complete Tumor Responders After Preoperative Chemoradiotherapy in Patients With Rectal Cancer: A Bi-institutional Validation Study. Annals of surgery 2014; Epub ahead of print. 


\section{CHAPTER 4}

\section{Magnetization Transfer Ratio: A potential biomarker for the assessment of post-radiation fibrosis in patients with rectal cancer}

Authors:

M.H. Martens,

D.M.J. Lambregts,

N. Papanikolaou,

L.A. Heijnen,

R.G. Riedl,

A. zur Hausen,

M. Maas,

G.L. Beets,

R.G.H. Beets-Tan

Investigative Radiology 2014 Jan;49(1):29-34 


\section{Abstract \\ Objectives}

Magnetization Transfer MRI (MT-MRI) utilizes differences in the magnetization interaction of free 'unbound' water-protons and macromolecular-bound protons. The aim of this study was to evaluate if the magnetization transfer ratio (MTR) may be used to identify fibrosis in rectal cancer patients treated with chemoradiotherapy.

\section{Materials and Methods}

This study was part of a rectal cancer imaging study, approved by the local institutional review board. Twenty-six patients, treated with neoadjuvant chemoradiotherapy, underwent a standard MRI including T2-weighted sequences and a diffusion-weighted sequence. An axially oriented MT-sequence was performed at the center of the (former) tumor location. Regions of interest (ROI) were manually drawn on the MT-MRI (with reference to the T2weighted and diffusion-weighted images), covering areas of either residual tumor, fibrosis or the normal or edematous rectal wall. Results were compared with histopathology. Differences in MTR between the four tissue types were analyzed and a receiver operating characteristics (ROC) curve was generated to assess the diagnostic potential.

\section{Results}

Thirty-eight ROIs were analyzed on the MT-MRI. The mean MTR of fibrosis was $37.7 \% \pm 2.7$, which was significantly higher than that of residual tumor $(29.6 \% \pm 4.2, p<0.001)$, normal rectal wall (30.3\% $\pm 4.7, p=0.003)$, or edematous rectal wall $(18.2 \% \pm 4.0, p<0.001)$. Use of MTR resulted in an area under the ROC curve of 0.96 , sensitivity of $88 \%$ and specificity of $90 \%$ for the diagnosis of fibrosis.

\section{Conclusion}

MTR can be used to discriminate post-radiation fibrosis from residual tumor and the normal rectal wall after chemoradiotherapy. MT-MRI could thus be a promising tool for the unsolved dilemma of interpreting post-radiation fibrosis in rectal cancer. 


\section{Introduction}

The general treatment for locally advanced rectal cancer is a long course of neo-adjuvant chemoradiotherapy (CRT) followed by surgical resection '. Nowadays, organ-saving treatment options (local excision or omission of surgery) for a selected group of good responding patients are being considered as good alternatives to standard resection ${ }^{2-6}$. Critical for a good outcome of organ-saving treatments is the selection of patients: which are the patients with residual tumor who still require surgical resection and which are patients with a sterilized, fibrotic tumor bed in whom standard resection may be omitted. The role of MRI in the workup of rectal cancer patients is well recognized, but its role as a response evaluation tool is not yet established. This is partly because morphological information from MRI has limited value for the interpretation of post-radiation fibrosis. Fibrosis in the irradiated tumor bed is known to contain residual tumor cells in approximately 50\% of the cases $^{7}$. To be on the safe side, radiologists tend to overstage the presence of residual tumor, resulting in false positive rates of $75-80 \%$ 8,9. Imaging techniques such as diffusion-weighted imaging (DWI) have shown promising results in detecting residual tumor. Nevertheless, the precise assessment of post-radiation fibrosis remains a challenge ${ }^{10}$, possibly explaining the relatively poor sensitivity to identify the complete responders with DWI reported by some authors (52-64\%) ${ }^{11}$. An additional tool that could quantify the fibrosis and exclude residual tumor could potentially improve treatment response evaluation.

Magnetization Transfer (MT) imaging is a technique that potentially has the ability to quantitatively assess fibrosis. The high collagen content of fibrosis reflects a high density of macromolecules. There are two different kind of protons: the free moving bulk water protons and the restricted protons that are bound to macromolecules. The latter cannot be directly imaged with MRI due to the ultra short T2 relaxation times they exhibit, which is responsible for selective signal loss. Magnetization exchange between free and bound protons is taking place through dipole-dipole interactions. When an MT pulse is applied, the bound protons are selectively saturated. The magnetization will then be transferred from the free protons to the bound protons, resulting in a decrease in MRI signal. The percentage of change of the signal intensity before and after the application of an MT pulse is defined as the 'magnetization transfer ratio' (MTR). In the absence of macromolecules there will be no change in signal and the MT ratio will remain low. In tissues with a predominantly macromolecular composition, such as fibrosis, the MTR will be high. Hence, the MTR may be used to discriminate between fibrotic and non-fibrotic tissues.

Currently, MT imaging is mainly used in time of flight MR angiography to improve the signal contrast between blood and other tissues ${ }^{12,13}$, and to improve the detection and characterization of white matter lesions in the brain in patients with multiple sclerosis ${ }^{14-16}$. More recently, MT imaging has been reported to be of value for the evaluation of cartilage in osteoarthritis ${ }^{17}$, the detection of prostate cancer ${ }^{18}$, the assessment of breast lesions ${ }^{19,20}$, and iron deposits in myocardial tissue ${ }^{21}$. Furthermore, MT imaging has proven feasible to assess 
the extent of fibrosis in pancreatic tumors and in Crohn's bowel strictures ${ }^{22,23}$, although the evidence so far is only available in preclinical studies.

We hypothesize that in patients with locally advanced rectal cancer the MTR of chemoradiotherapy-induced fibrosis on restaging MRI is higher than the MTR of other tissues (e.g. tumor, normal rectal wall, and edema). The purpose of this study was to evaluate whether it is feasible to differentiate fibrosis from residual tumor in patients with locally advanced rectal cancer treated with preoperative chemoradiotherapy using magnetization transfer imaging.

\section{Materials and methods}

\section{Patients}

Thirty-five consecutive patients with locally advanced rectal cancer were considered for inclusion in this prospective study between December 2011 and July 2012. The study was conducted as part of a rectal cancer imaging study, which was approved by the local institutional review board. Inclusion criteria were: 1) biopsy proven rectal cancer, 2) locally advanced disease (staged with MRI as cT3-4, involved mesorectal fascia, and/or cN+), and 3) treated with a long course of neoadjuvant therapy (capecitabine and/or oxaliplatin with 50.4 Gy, or 5x5 Gy with Rapamycin) followed by surgery. Nine patients did not receive surgery and were excluded. This left a total study population of 26 eligible patients. Twenty-two patients were male and four were female. The median age was 66.0 years (range 49-80). All patients underwent a restaging MRI including MTR approximately 8 weeks after completion of neoadjuvant therapy.

\section{MR Imaging}

MR imaging was performed at 1.5 T using a phased array body coil (Intera, Philips Medical Systems, Best, the Netherlands). Patients were placed in feet first supine position and received an intravenous injection of $20 \mathrm{mg}$ of butylscopolamine (Buscopan ${ }^{\circledR}$,Boehringer Ingelheim bv, Ingelheim, Germany) to minimize peristaltic movement. Patients did not receive any bowel-preparation. The MR protocol consisted of standard T2-weighted (T2W) fast spin-echo sequences in three orthogonal planes (TR/TE 8456/130 ms, 90 flip angle, 25

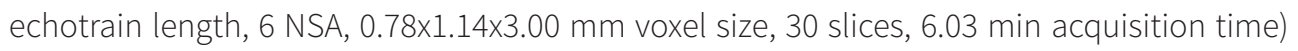
and an axial diffusion weighted sequence (spectral presaturation with inversion recovery, b-values 0, 500, and $1000 \mathrm{~s} / \mathrm{mm} 2$, TR/TE 4971/70 ms, 55 EPI factor, 5 NSA, 1.82×2.31×5.00 mm voxel size, 24 slices, 5.33 min acquisition time). One axially oriented slice of the magnetization transfer contrast sequence was acquired in the center of the area suspicious for residual tumor as visualized on the post-treatment T2W sequence. Parameters of the MT sequence were: 2D FFE, TR/TE 32/4.6, $12^{\circ}$ flip angle, 2 NSA, 1.17×1.47×5.00 mm voxel size, 1 slice, 10 $\mathrm{s}$ acquisition time. The MT-pulse was an on-resonance composite pulse and consisted of 4 
rectangular pulses $\left(90^{\circ}-\times 90^{\circ} \times 90^{\circ} \times 90^{\circ}-x\right)$ with a duration of $2 \mathrm{~ms}\left(0.5 \mathrm{~ms}\right.$ for each $90^{\circ}$ pulse). All axial sequences were angled in identical planes, perpendicular to the tumor axis.

\section{MR Evaluation}

MTR measurements were performed using Weasis Medical Viewer 1.1.2, an open-source post-processing tool. MTR maps were generated by applying the following formula on a pixel-by-pixel basis: MTR=100x(SI ${ }_{\text {off }}-\mathrm{SI}_{\text {on }}$ )/SI off , where $\mathrm{SI}_{\text {off }}$ and $\mathrm{SI}_{\text {on }}$ represent the signal intensity with the MT pulse turned off and on, respectively (Figure1). The (T2-weighted) MR images obtained before onset of treatment were used as a reference to determine the initial location and circumferential growth pattern of the tumor. Two readers in consensus (MHM and DMJL) with respectively one and four years of specific expertise in reading MRI of rectal cancer) manually placed regions of interest (ROI).

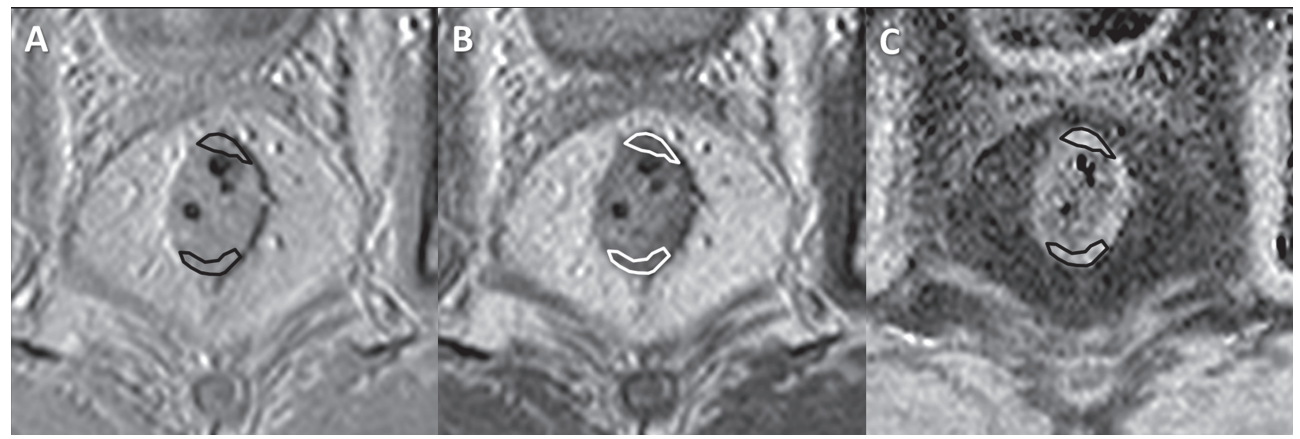

\section{Figure 1.}

Axial 2D FFE images obtained before (A) and after (B) application of the magnetization transfer pulse. A magnetization transfer ratio (MTR) map was calculated (C). This example shows an area of fibrosis at the ventral side, visible as high signal on the MTR map. At the dorsal side an area of normal rectal wall is visualized, showing moderate to high signal on the MTR map. The MTR of the area with fibrosis was significantly higher (40.4\%) than that of the normal rectal wall (30.6\%).

ROIs were manually drawn on the MTR-maps covering areas of [1] residual tumor, [2] fibrosis, [3] normal rectal wall, or [4] edematous rectal wall, as identified with reference to the corresponding T2-weighted and the diffusion-weighted (b1000) images. Areas of residual tumor were defined as a wall thickening or mass showing isointense signal on T2W-MRI and corresponding high signal on high b-value DWI. Areas of fibrosis were defined as a fully hypointense wall thickening on T2W-MRI with no corresponding high signal on DWI. A normal rectal wall was defined as a two-layered rectal wall on T2W-MRI consisting of an isointense mucosal layer and hypointense muscularis without any visible wall thickening or high signal on DWI. An edematous rectal wall was defined as a hyperintense thickened bowel wall on T2W-MRI with corresponding low signal on DWI. Depending on the different tissues present in the one slice of MTR, a total of 1 to 3 ROIs were included per patient. The mean MTR was calculated for each ROI (Figure 2). 


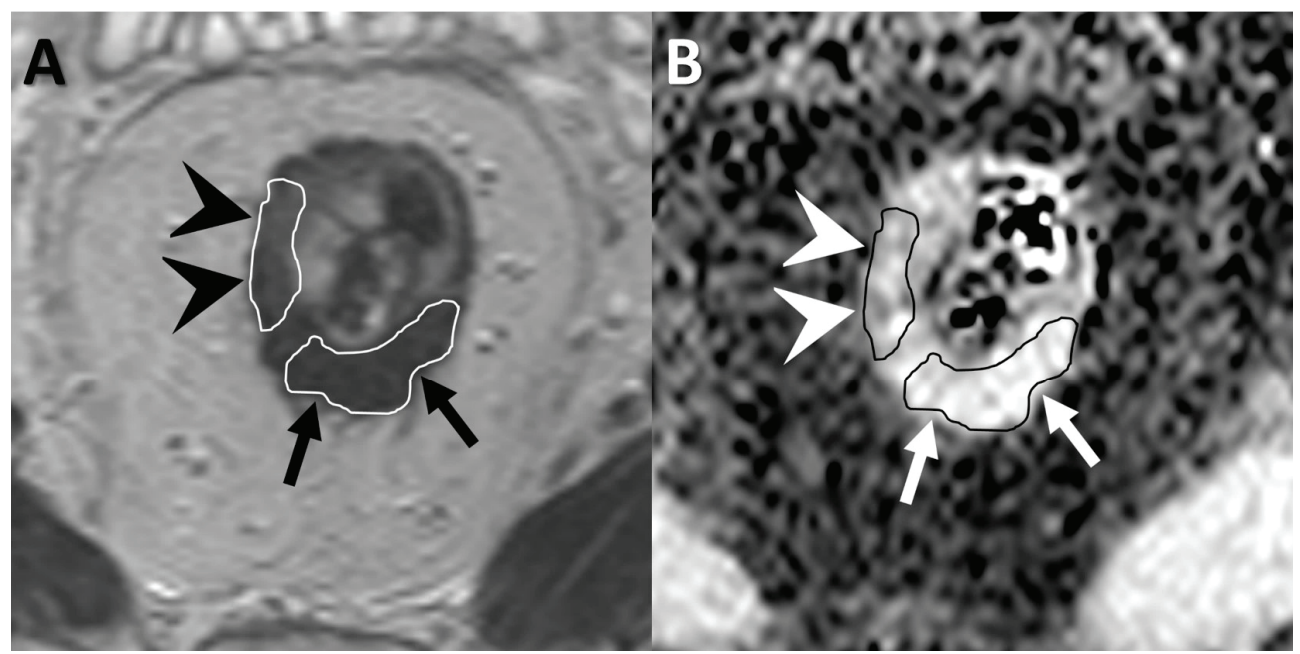

\section{Figure 2.}

Axial T2-weighted image after neo-adjuvant chemoradiotherapy (A), and the corresponding magnetization transfer ratio (MTR) map after chemoradiotherapy (B). Regions of interest are determined on the MTR map with reference to their morphologic appearance on the T2-weighted images and histopathologic correlation. At the dorsal side, histopathology showed extensive fibrosis (arrows) The mean MTR of the fibrosis was $41.9 \%$. On the right lateral side residual tumor was present at histopathology (arrow heads), in this ROI the mean MTR was $25.6 \%$.

\section{Histopathological correlation}

For each patients the results of the restaging MRI including the MT sequence were compared with histological findings of the resection specimen.The histopathological assessment of the surgical resection specimen was performed according to the method of Quirke et al ${ }^{24}$. In eleven patients an additional staining with Sirius red (specific for fibrosis) was performed to allow accurate correlation between the MTR map and histopathology.

\section{Statistical analyses}

Statistical analyses were performed using the Statistical Package for the Social Sciences (SPSS, version 18.0, Inc, Chicago, Illinois, USA). One-way ANOVA was used to test differences in MTR between the four predefined tissue types (tumour, fibrosis, normal rectal wall and edema). A Tukey post-hoc test was used to compare all means of the MTR. A p-value of less than 0.05 was considered statistically significant. The diagnostic potential of the MTR to diagnose fibrosis was assessed using receiver operating characteristic (ROC) curves analysis and the area under the ROC-curve was calculated. The optimal MTR cut-off value was determined based on the point nearest to the upper left corner of the ROC curve. Corresponding sensitivity, specificity, positive predictive value (PPV) and negative predictive value (NPV) with 95\% confidence intervals (Cl) were calculated. 


\section{Results}

\section{Patient characteristics}

All patients underwent surgical resection: 12 underwent a low anterior resection, 6 an abdominoperineal resection, 1 a Hartmann's procedure, 5 transanal endoscopic microsurgery, and 2 more extended surgery. At histopathology, 8 patients had an ypT0, 2 урт1, 8 урт2, 7 урт3, and 1 yрT4 stage.

\section{MT ratios}

A total number of 38 ROls was analyzed, ranging in size from 15 to 298 mm² $^{2}$ mean 103.8 \pm 65.5 ). The mean MTRs for the four tissue types are displayed in Table 1 . The mean MTR of fibrosis was $37.7 \%$, which was significantly higher than the mean MTR of residual tumor (29.6\%, $p<0.001)$, the normal rectal wall $(30.2 \%, p=0.003)$ and the edematous rectal wall (18.2\%, $p<0.001)$. There was no significant difference in the mean MTR between residual tumor and normal rectal wall ( $p=0.980)$. The mean MTR of edematous rectal wall $(18.2 \%)$ was significantly lower than that of both residual tumor and the normal rectal wall $(p<0.001)$.

Table 1. The mean magnetization transfer ratio for fibrosis, residual tumor, normal rectal wall, and edematous rectal wall.

\begin{tabular}{|c|c|}
\hline Tissue as defined on T2W-MRI and histopathology ( $n^{\circ}$ of ROIs) & Mean MTR ( \pm SD) \\
\hline Fibrosis $(n=8)$ & $37.7(2.7)$ \\
\hline Residual tumor ( $\mathrm{n}=13$ ) & $29.6(4.2)$ \\
\hline Normal rectal wall $(n=9)$ & $30.3(4.7)$ \\
\hline Edematous rectal wall $(n=8)$ & $18.2(4.0)$ \\
\hline
\end{tabular}

$\mathrm{T} 2 \mathrm{~W}-\mathrm{MRI}=\mathrm{T} 2$ weighted MRI, MTR= magnetization transfer ratio, $\mathrm{SD}=$ standard deviation .

\section{Histopathological correlation}

One of the MR readers was always present during histopathological examination in order to ensure accurate correlation between the imaging findings and corresponding histopathology. Histopathology (including the Sirius red staining specific for fibrosis) confirmed the presence of residual tumor in all patients with ROIs referring to residual tumor, and extensive fibrosis without tumor cells in all patients with ROIs referring to fibrosis. In the eleven patients in whom additional Sirius red staining was performed on the total rectal wall and surrounding mesorectal fat the localization and extension of the fibrotic areas correlated well with the MTR maps. Figure 3 shows an example of the correlation between the MTR measurements and histopathology including Sirius red staining. 

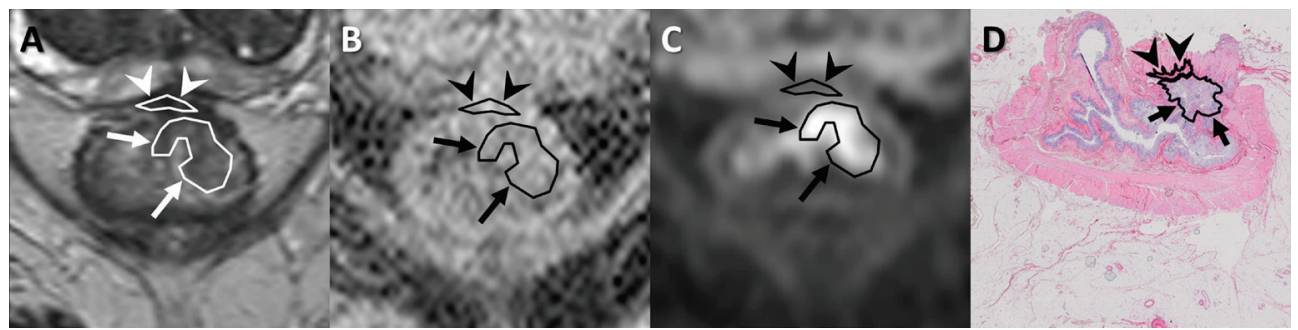

Figure 3.

Axial T2-weigthed image (A) and corresponding magnetization transfer ratio (MTR) map (B), b1000 diffusionweighted image (C), and whole mount section with Sirius red staining (no magnification) (D) of a patient with a CT3N1 rectal tumor that was treated with a long course of neo-adjuvant chemoradiotherapy. Regions of interest were determined on the MTR map with reference to the T2-weighted images and diffusion weighted images. The mean MTR of residual tumor (arrows) was $23.5 \%$ and the mean MTR of fibrosis (arrowheads) was $34.7 \%$. At histopathology, these areas corresponded with residual tumor (arrows) and fibrosis (arrowheads), respectively.

\section{Diagnostic performance}

The area under the ROC curve (Figure 4) for differentiating between fibrosis and non-fibrotic tissues (e.g. residual tumor and normal or edematous rectal wall) based on the MTR was 0.96 (95\% $\mathrm{Cl}$ 0.91-1.00). When applying a cut-off MTR value of $34.7 \%$, a sensitivity of $88 \%(7 / 8,95 \%$ Cl $47-99 \%)$, specificity of $90 \%(27 / 30,95 \% \mathrm{Cl} 72-97 \%)$, positive predictive value of $70 \%$ (7/10, $95 \% \mathrm{Cl} 35-92 \%)$, and negative predictive value of $96 \%$ (27/28, 95\% Cl 80-100\%) was obtained.

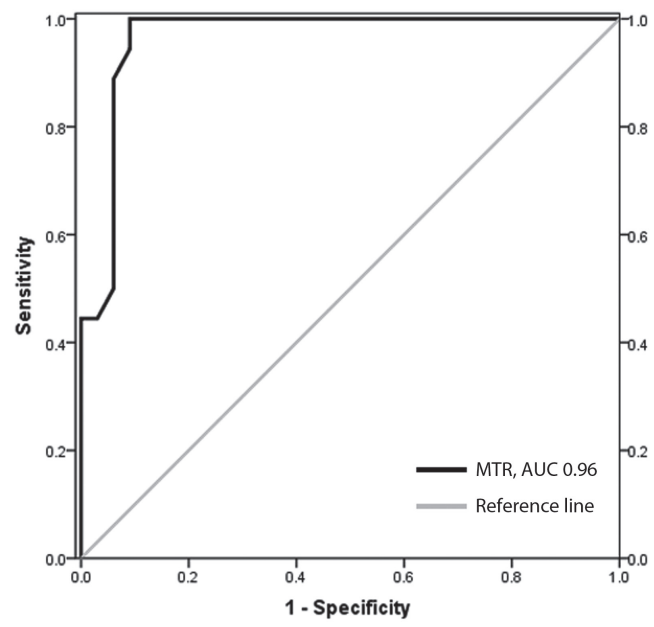

\section{Figure 4.}

Receiver operating characteristics curve for differentiating between fibrosis and non-fibrotic tissues using the magnetization transfer ratio (MTR). The area under the curve was $0.96(95 \% \mathrm{Cl} 0.91-1.00)$

\section{Discussion}

The purpose of this study was to evaluate whether it is feasible to differentiate fibrosis from residual tumor in patients with locally advanced rectal cancer treated with neo-adjuvant chemoradiotherapy using magnetization transfer MR imaging. The MT ratio for the different tissue types (residual tumor, fibrosis, normal rectal wall, and edematous rectal wall) was 
calculated in 26 patients treated with chemoradiotherapy. Our hypothesis was that the MTR of CRT-induced fibrosis is higher than that of other tissues. The results of our study support this hypothesis and show that MT imaging can differentiate fibrosis from residual tumor and the normal or edematous rectal wall with a high AUC of 0.96 .

To our knowledge, this is the first study to assess the potential of MTR to assess bowel fibrosis in a human study population. The results are in line with the findings of a previous study using an animal model; Adler et al. investigated whether fibrotic changes in bowel strictures in a rat's model of Crohn's disease were associated with higher MT ratio's. They found that the MTR increased in areas with fibrosis as found at histology and concluded that MT imaging is sensitive to detect bowel fibrosis ${ }^{22}$. In addition to bowel fibrosis, Li et al. showed that areas of high MTR also correlated with areas of high fibrotic levels at histology in a mouse model with pancreatic cancer cell lines ${ }^{23}$. Similarly, MT ratios have also been reported to correlate with fibrosis in the breast ${ }^{25}$ and in the brain in patients with cirrhotic liver disease ${ }^{26}$. Conversely, MTR has by different authors been shown to be of limited value for the assessment of fibrosis in the liver ${ }^{27,28}$. A possible explanation for the lack of increased MTR in liver fibrosis could be the diffuse pattern of disease. In the liver, fibrosis develops heterogeneously with the formation of fibrotic bands, while in the bowel fibrosis occurs in a focal pattern instead of diffuse. This could explain the difficulty of MT imaging to detect fibrosis in the liver.

In patients with rectal cancer, the assessment of fibrosis is a particularly relevant issue, since there is increasing evidence showing that organ saving treatment for patients with a true complete response (sterilized fibrosis in the tumor bed) is a good alternative to standard resection with less morbidity and without compromising outcome ${ }^{2,3}$. Crucial in decision making is the selection of the patients with a complete response. Morphological assessment of standard T2W-images alone is insufficiently accurate for differentiating between mere fibrosis and fibrosis with residual tumor (cells), with reported sensitivities of only $20-42 \%$ $8,9,29$. Hence, objective information in addition to subjective morphology may be useful to improve the performance in assessing the response to CRT. In this regard, the potential value of functional imaging techniques such as FDG-PET ${ }^{30}$, dynamic contrast enhanced MRI ${ }^{31}$, and diffusion-weighted MR imaging (DWI) ${ }^{11,32,33}$ have recently been investigated, because these techniques can provide additional functional information reflecting the tissue metabolic behavior, perfusion characteristics, and cellular tissue structure. The main endpoint of these previous studies was the identification of residual tumor and its distinction from a complete tumor response. For example, DWI has been shown to be a very sensitive technique to detect areas of vital residual tumor after chemoradiotherapy ${ }^{11,33-35}$, yet for the qualitative (visual) interpretation of DWI in the assessment of a complete tumor response, the reported sensitivities remain relatively low, ranging from $52-64 \%{ }^{11}$. The visual interpretation of DWI is generally performed in combination with T2W images for anatomical reference. In this setting, extensive fibrosis on $\mathrm{T} 2 \mathrm{~W}$ images often results in an overestimation of residual tumor despite the absence of restricted diffusion (indicating tumor) on DWI. Studies that have 
applied a quantitative assessment of DWI - i.e. measuring the apparent diffusion coefficient (ADC) - have also reported conflicting results. Whereas some authors claim significantly different ADC values between patients with residual tumor and patients with a complete response ${ }^{34-36}$, others found no differences in ADC between the two groups ${ }^{32,37}$. A possible explanation could be the method of image processing. With the traditional 2 dimensional (ROI) data extraction of ADC, the mean ADC over all pixels is generated. This mean ADC value does not take regions consisting of fibrosis into account. In particular, in the complete response group, results may have been influenced by the contribution of both normal or edematous rectal wall tissue components as well as areas of fibrosis within the ROI. This may explain why in some studies the measured ADC values were not found to be useful in discriminating between the complete response and residual tumor group, in whom similar fibrotic areas may be present within the measured ROIs ${ }^{32,37}$. In contrast, MT-MRI specifically focuses on quantifying the fibrotic tissue compound. The knowledge that sterilized fibrosis (complete tumor regression) has a significantly different MTR than all other tissues is even more interesting for a precise identification of the true complete responder, who can be considered for organ saving treatments. When further research confirms our findings, MTMRI could be complementary to DWI and T2W and information on the response after CRT will be more accurate and comprehensive. Our current study was performed at 1.5 Tesla MRI, but results should in theory also be reproducible at higher field strengths. Similar results have been shown for rectal cancer staging using $1.5 \mathrm{~T}$ and 3.0T MRI $(37,38)$. Regarding the MT sequence, it has been shown that for abdominal MT imaging, MTR values vary only slightly between 1.5T and 3.0T MRI38.

Our study was regarded as a 'proof of principle' study. As such, there are some limitations to the study design. Although the presence of residual tumor and/or fibrosis was histologically confirmed for each patient, we did not perform a whole-mount correlation for the MTR measurements in each single patient. Secondly, only one slice of the MT sequence was currently performed. Now that we have shown the potential value of MT-MRI for assessing fibrosis, the next step would be to perform multislice MT-MRI covering the whole tumor volume (acquisition time \pm 4 minutes) and compare this with whole mount sections of the resection specimen.

In conclusion, this study has shown that magnetization transfer MR imaging may be useful in differentiating fibrosis from all other tissues such as residual tumor, normal rectal wall, and edematous rectal wall in patients with locally advanced rectal cancer treated with CRT. MT-MRI therefore is promising in addressing the yet unsolved dilemma of interpreting postradiation fibrosis. The preliminary results are such that further research with a large patient cohort is warranted. 


\section{References}

1. Glimelius B, Oliveira J. Rectal cancer: ESMO clinical recommendations for diagnosis, treatment and follow-up. Ann Oncol 2009; 20 Suppl 4: 54-6.

2. Maas M, Beets-Tan RG, Lambregts DM, et al. Wait-and-see policy for clinical complete responders after chemoradiation for rectal cancer. J Clin Oncol 2011; 29(35): 4633-40.

3. Habr-Gama A, Perez RO, Proscurshim I, et al. Patterns of failure and survival for nonoperative treatment of stage c0 distal rectal cancer following neoadjuvant chemoradiation therapy. Journal of gastrointestinal surgery: official journal of the Society for Surgery of the Alimentary Tract 2006; 10(10): 1319-28; discussion 28-9.

4. Lezoche G, Baldarelli M, Guerrieri M, et al. A prospective randomized study with a 5-year minimum follow-up evaluation of transanal endoscopic microsurgery versus laparoscopic total mesorectal excision after neoadjuvant therapy. Surgical endoscopy 2008; 22(2): 352-8.

5. Borschitz T, Wachtlin D, Mohler M, Schmidberger H, Junginger T. Neoadjuvant chemoradiation and local excision for T2-3 rectal cancer. Ann Surg Oncol 2008; 15(3): 712-20.

6. Glynne-Jones R, Hughes R. Critical appraisal of the 'wait and see' approach in rectal cancer for clinical complete responders after chemoradiation. Br J Surg 2012; 99(7): 897-909.

7. Vliegen RF, Beets GL, Lammering $G$, et al. Mesorectal fascia invasion after neoadjuvant chemotherapy and radiation therapy for locally advanced rectal cancer: accuracy of MR imaging for prediction. Radiology 2008; 246(2): 454-62.

8. Chen CC, Lee RC, Lin JK, Wang LW, Yang SH. How accurate is magnetic resonance imaging in restaging rectal cancer in patients receiving preoperative combined chemoradiotherapy? Dis Colon Rectum 2005; 48(4): 722-8.

9. Suppiah A, Hunter IA, Cowley J, et al. Magnetic resonance imaging accuracy in assessing tumour down-staging following chemoradiation in rectal cancer. Colorectal Dis 2009; 11(3): 249-53.

10. Klauss M, Gaida MM, Lemke A, et al. Fibrosis and pancreatic lesions: counterintuitive behavior of the diffusion imaging-derived structural diffusion coefficient d. Invest Radiol 2013; 48(3): 129-33.

11. Lambregts DM, Vandecaveye V, Barbaro B, et al. Diffusion-weighted MRI for selection of complete responders after chemoradiation for locally advanced rectal cancer: a multicenter study. Ann Surg Oncol 2011; 18(8): 2224-31.

12. Nezafat R, Han Y, Peters DC, et al. Coronary magnetic resonance vein imaging: imaging contrast, sequence, and timing. Magn Reson Med 2007; 58(6): 1196-206.

13. Henkelman RM, Stanisz GJ, Graham SJ. Magnetization transfer in MRI: a review. NMR Biomed 2001; 14(2): 57-64.

14. Karampekios S, Papanikolaou N, Papadaki E, et al. Quantification of magnetization transfer rate and native $\mathrm{T} 1$ relaxation time of the brain: correlation with magnetization transfer ratio measurements in patients with multiple sclerosis. Neuroradiology 2005; 47(3): 189-96.

15. Papanikolaou N, Papadaki E, Karampekios S, et al. T2 relaxation time analysis in patients with multiple sclerosis: correlation with magnetization transfer ratio. Eur Radiol 2004; 14(1): 115-22.

16. Papanikolaou N, Maniatis V, Pappas J, Roussakis A, Efthimiadou R, Andreou J. Biexponential T2 relaxation time analysis of the brain: correlation with magnetization transfer ratio. Invest Radiol 2002; 37(7): 363-7. 
17. Wachsmuth L, Juretschke HP, Raiss RX. Can magnetization transfer magnetic resonance imaging follow proteoglycan depletion in articular cartilage? MAGMA 1997; 5(1): 71-8.

18. Kumar V, Jagannathan NR, Kumar R, et al. Evaluation of the role of magnetization transfer imaging in prostate: a preliminary study. Magn Reson Imaging 2008; 26(5): 644-9.

19. Bonini RH, Zeotti D, Saraiva LA, et al. Magnetization transfer ratio as a predictor of malignancy in breast lesions: preliminary results. Magn Reson Med 2008; 59(5): 1030-4.

20. Leong CS, Daniel BL, Herfkens RJ, et al. Characterization of breast lesion morphology with delayed 3DSSMT: an adjunct to dynamic breast MRI. J Magn Reson Imaging 2000; 11(2): 87-96.

21. Papanikolaou N, Ghiatas A, Kattamis A, Ladis C, Kritikos N, Kattamis C. Non-invasive myocardial iron assessment in thalassaemic patients. T2 relaxometry and magnetization transfer ratio measurements. Acta Radiol 2000; 41(4): 348-51.

22. Adler J, Swanson SD, Schmiedlin-Ren P, et al. Magnetization transfer helps detect intestinal fibrosis in an animal model of Crohn disease. Radiology 2011; 259(1): 127-35.

23. Li W, Zhang Z, Nicolai J, Yang GY, Omary RA, Larson AC. Magnetization transfer MRI in pancreatic cancer xenograft models. Magn Reson Med 2012; 68(4): 1291-7.

24. Quirke P, Durdey P, Dixon MF, Williams NS. Local recurrence of rectal adenocarcinoma due to inadequate surgical resection. Histopathological study of lateral tumour spread and surgical excision. Lancet 1986; 2(8514): 996-9.

25. Matsushima S, Sasaki F, Kinosada Y, et al. Saturation transfer ratio imaging in invasive ductal carcinomas of the breast. Magn Reson Imaging 2000; 18(4): 379-85.

26. Miese F, Kircheis G, Wittsack HJ, et al. 1H-MR spectroscopy, magnetization transfer, and diffusion-weighted imaging in alcoholic and nonalcoholic patients with cirrhosis with hepatic encephalopathy. AJNR Am J Neuroradiol 2006; 27(5): 1019-26.

27. Kim H, Booth CJ, Pinus AB, et al. Induced hepatic fibrosis in rats: hepatic steatosis, macromolecule content, perfusion parameters, and their correlations--preliminary MR imaging in rats. Radiology 2008; 247(3): 696-705.

28. Rosenkrantz AB, Storey P, Gilet AG, et al. Magnetization transfer contrast-prepared MR imaging of the liver: inability to distinguish healthy from cirrhotic liver. Radiology 2012; 262(1): 136-43.

29. Dresen RC, Beets GL, Rutten HJ, et al. Locally advanced rectal cancer: MR imaging for restaging after neoadjuvant radiation therapy with concomitant chemotherapy. Part I. Are we able to predict tumor confined to the rectal wall? Radiology 2009; 252(1): 71-80.

30. Capirci C, Rubello D, Chierichetti F, et al. Restaging after neoadjuvant chemoradiotherapy for rectal adenocarcinoma: role of F18-FDG PET. Biomedicine \& pharmacotherapy = Biomedecine \& pharmacotherapie 2004; 58(8): 451-7.

31. Gollub MJ, Gultekin DH, Akin O, et al. Dynamic contrast enhanced-MRI for the detection of pathological complete response to neoadjuvant chemotherapy for locally advanced rectal cancer. Eur Radio/ 2011; 22(4): 821-31.

32. Curvo-Semedo L, Lambregts DM, Maas M, et al. Rectal cancer: assessment of complete response to preoperative combined radiation therapy with chemotherapy--conventional MR volumetry versus diffusion-weighted MR imaging. Radiology 2011; 260(3): 734-43.

33. Kim SH, Lee JM, Hong SH, et al. Locally advanced rectal cancer: added value of diffusion-weighted MR imaging in the evaluation of tumor response to neoadjuvant chemo- and radiation therapy. Radiology 2009; 253(1): 116-25. 
34. Song I, Kim SH, Lee SJ, Choi JY, Kim MJ, Rhim H. Value of diffusion-weighted imaging in the detection of viable tumour after neoadjuvant chemoradiation therapy in patients with locally advanced rectal cancer: comparison with T2 weighted and PET/CT imaging. Br J Radiol 2011; 85(1013): 577-86.

35. Lambrecht M, Deroose C, Roels S, et al. The use of FDG-PET/CT and diffusion-weighted magnetic resonance imaging for response prediction before, during and after preoperative chemoradiotherapy for rectal cancer. Acta Oncol 2010; 49(7): 956-63.

36. Kim SH, Lee JY, Lee JM, Han JK, Choi BI. Apparent diffusion coefficient for evaluating tumour response to neoadjuvant chemoradiation therapy for locally advanced rectal cancer. Eur Radiol 2010; 21(5): 987-95.

37. Sun YS, Zhang XP, Tang L, et al. Locally advanced rectal carcinoma treated with preoperative chemotherapy and radiation therapy: preliminary analysis of diffusion-weighted MR imaging for early detection of tumor histopathologic downstaging. Radiology 2010; 254(1): 170-8.

38. Martirosian P, Boss A, Deimling M, et al. Systematic variation of off-resonance prepulses for clinical magnetization transfer contrast imaging at 0.2, 1.5, and 3.0 tesla. Invest Radiol 2008; 43(1): 16-26. 


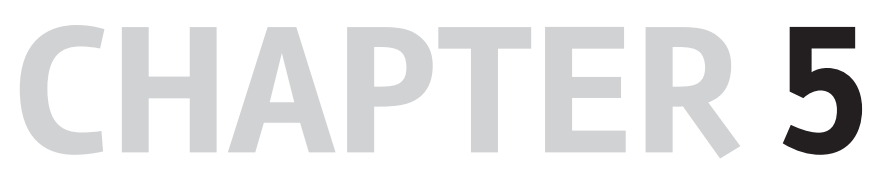

Magnetization Transfer Imaging to assess tumor response
after chemo-radiotherapy in rectal cancer

Authors:

M.H. Martens,

D.M.J. Lambregts,

N. Papanikolaou,

S. Alefantinou,

M. Maas,

G.C. Manikis,

K. Marias,

R.G. Riedl,

G.L. Beets,

R.G.H. Beets-Tan

European Radiology 2015 Jun 12. [Epub ahead of print] 


\section{Abstract}

\section{Purpose}

Single-slice magnetization transfer (MT) imaging has shown promising results to evaluate post-radiation fibrosis. Aim of study was to evaluate the value of multislice MT imaging to assess tumor response after chemoradiotherapy by comparing magnetization transfer ratios (MTR) with histopathological tumor regression grade (TRG).

\section{Materials and Methods}

30 patients with locally advanced rectal cancer (cT3-4 and/or cN2) underwent restaging MRI routinely performed 8 weeks post-chemoradiotherapy, including multislice MT-sequence, covering entire tumor bed. Two independent readers delineated regions of interest on MTR maps, covering all potential remaining tumor and fibrotic areas. Mean MTR and histogram parameters (minimum, maximum, median, standard deviation, skewness, kurtosis, and 5-30-70-95 th percentiles) were calculated. Reference standard was histological TRG1-2 (good response) and TRG3-5 (poor response).

\section{Results}

24/30 patients were male; mean age was $67.7 \pm 10.8$ years. Mean MTR rendered AUCs of 0.65 (reader1) and 0.87 (reader2) to differentiate between TRG1-2 versus TRG3-5. Best results were obtained for $95^{\text {th }}$ percentile (AUC 0.75- 0.88). Interobserver agreement was moderate (ICC 0.50) for mean MTR and good (ICC 0.80) for $95^{\text {th }}$ percentile.

\section{Conclusion}

MT imaging is a promising tool to assess tumor response post-chemoradiotherapy in rectal cancer. Particularly $95^{\text {th }}$ percentile results in AUCs up to 0.88 to discriminate a good tumor response. 


\section{Introduction}

Assessment of response after chemoradiotherapy (CRT) in patients with rectal cancer has become increasingly relevant. Until recently, chemoradiotherapy followed by surgical resection of the tumor was the standard, irrespective of the response to treatment ${ }^{1}$. However organ-preserving treatments in carefully selected patients who show a clinically complete or near-complete response are now considered potential alternatives to resection, albeit still controversial. Alternative options include a local excision for a small tumor remnant ${ }^{2}$ and a non-operative management ("wait-and-see" policy) in case of a complete tumor response $e^{3-5}$. Critical in these organ-preserving treatments is the availability of tools to accurately select the suitable patients. In reported studies so far this has mainly been done by clinical examination and endoscopy ${ }^{6}$. A restaging MRI 8 weeks after chemoradiotherapy has become standard in many centers because MRI has proven valuable in assessing tumor downsizing, identifying tumor regression from initially invaded structures and thereby altering the surgical approach ${ }^{7}$. However, one of the main challenges of MRI remains the interpretation of post-radiation fibrosis ${ }^{8}$. With morphological MRI it is very difficult to discriminate between fibrosis with and without tumor remnants ${ }^{9}$, explaining the poor sensitivity of $19 \%$ reported for MRI in a recent meta-analysis for discriminating complete response ( $P C R)$ from residual tumor $^{10}$. Quantifying signal intensities on T2-weighted MRI has been suggested to be helpful: signal intensity decreases more after chemoradiation in complete responders with a more homogeneous signal distribution compared to patients with residual tumor. So far this approach has only been addressed by a single report ${ }^{11}$. Functional imaging techniques such as diffusion-weighted imaging and dynamic contrast-enhanced MRI have shown promising results in detecting residual tumor after CRT in different reports ${ }^{12-14}$, but these techniques do not specifically target fibrosis.

In recent reports it was shown that 'Magnetization Transfer' (MT) imaging may also be a helpful tool to assess bowel fibrosis ${ }^{15-17}$. MT-imaging is a technique that utilizes differences in the magnetization interaction of free, 'unbound' water-protons and macromolecularbound protons. After applying an MT pulse, there will be a transfer of magnetization from free water-protons towards macromolecular-bound water protons. This transfer can only occur when macromolecular-bound water protons are present within a given tissue. In tissues that are rich in macromolecules (which is typically the case in fibrosis due to the presence of collagen), the transfer of magnetization - expressed as the "Magnetization Transfer Ratio" or MTR - will be high. In a recent proof of principle study of 26 patients with rectal cancer who were treated with preoperative CRT, the MTR of histopathologically proven areas of fibrosis was significantly higher than that of areas of residual tumor, indicating that there might be a potential benefit in adding MT-imaging to a rectal cancer imaging protocol after CRT ${ }^{16}$. In this report, only a single slice of the MT-sequence was acquired, which did not cover (and is therefore not representative of) the entire tumor area. As such it is difficult to assess the value of MT imaging to evaluate the overall tumor response. Moreover, only mean MTR values were 
assessed, which provide a rough estimation of the MTR of a given area of interest, but does not take into account the heterogeneity of the tumor and fibrosis.

The aim of the current study therefore was to evaluate the potential value of multislice MTimaging of the whole tumor volume for assessing tumor response after CRT by comparing the magnetization transfer ratios with the tumor regression grade (TRG) as assessed at histopathology. A second goal was to assess the added benefit of performing histogram analyses as compared to evaluation of only mean MTR values.

\section{Materials and Methods}

\section{Patients}

This study was performed retrospectively. According to our country's national law, institutional review board approval was not required. All patients were informed that their anonymized data could be used for research purposes, to which none of the study patients objected. Between November 2012 and June 2014, all consecutive patients with locally advanced rectal cancer were considered for inclusion. Inclusion criteria were (1) biopsy proven rectal adenocarcinoma, (2) locally advanced disease (cT3/4, and/or N2) without distant metastasis, (3) neoadjuvant chemoradiation, (4) availability of a restaging MRI including the MT-sequence, (5) resection within 2 months after restaging MRI or in case of non-operative treatment and follow-up in patients with a clinical complete response - a disease-free follow-up period of $>1$ year.

\section{MR Imaging}

MR imaging was performed at 1.5T (Ingenia, Philips Medical Systems, Best, the Netherlands) using a phased array body coil. Patients were placed in feet-first supine position and did not receive any bowel preparation. To minimize peristaltic movements, patients received an intravenous bolus injection of 20 mg of butylscopolamine (Buscopan, Boehringer Ingelheim BV, Ingelheim, Germany). The routine clinical MR protocol included standard T2-weighted (T2W) fast spin-echo sequences in 3 planes (sagittal, axial, and coronal). Additionally an axial MTR sequence was performed with the following parameters: Multislice fast field echo, dynamic (simultaneous acquisition of images with and without MT pulse in one sequence), interleaved two-dimensional, TR/TE 50/4.6, $12^{\circ}$ flip angle, 2 NSA, 1.17×1.47×3.00 mm voxel size, 10 slices, $300 \times 239$ mm field of view, $256 \times 162$ matrix, receiver bandwidth $147 \mathrm{~Hz} /$ pixel, 5:02 minutes acquisition time. The MT-pulse was an on-resonance composite pulse and consisted of 4 rectangular pulses $\left(90^{\circ}-\times 90^{\circ} \times 90^{\circ} \times 90^{\circ}-x\right)$ with a duration of $2 \mathrm{~ms}(0.5 \mathrm{~ms}$ for each $90^{\circ}$ pulse) and a bandwidth of $2083 \mathrm{~Hz}$. Delay between MT pulse and the following excitation was 3.2 ms. The axial T2-weighted and MT sequences were angulated in identical planes, perpendicular to the (former) tumor axis as defined on sagittal MRI. 


\section{Image evaluation and calculation of the Magnetization Transfer Ratio (MTR) parameters.}

Image assessment was performed on the restaging MRI routinely performed 8 weeks after completion of CRT. Magnetization Transfer Ratio maps were generated using a freely distributed software tool (MANGO; Multi-image Analysis GUI, version 2.6, Research Imaging Institute, University of Texas Health Science Center, San Antonio, USA). MTR maps were generated using the following formula on a voxel-by-voxel basis: MTR=100x ( I $\left._{\text {off }}-S_{\text {on }}\right)$ / $\mathrm{SI}_{\text {ofp }}$ where $\mathrm{SI}_{\text {off }}$ and $\mathrm{SI}_{\text {on }}$ represent the signal intensity with the MT pulse turned off and on, respectively. Two independent readers with respectively 6 (DMJL) and 3 years (MHM) of specific expertise in rectal cancer imaging manually delineated free-hand Regions of Interest (ROIs) on the MTR maps on each individual tumor-containing slice. The corresponding T2weighted images, as well as the primary staging MRIs were at the readers' disposal as an anatomical reference and to determine the primary tumor location (Figure 1). On every slice, the entire area of potential residual tumor was included within the ROI, including any potential viable tumor remnants (iso-intense mass or wall thickening), as well as fibrosis (hypo-intense wall thickening). The two readers were blinded to each other's results and histopathology outcomes. Histogram analyses were performed using in-house software, developed in the programming environment $\mathrm{R}$ language ${ }^{18}$. The MTR measurements were imported and the following normalized histogram parameters were calculated automatically: minimum, maximum, median, standard deviation, skewness (asymmetry of distribution), kurtosis (width of peak of distribution), and $5^{\text {th }}, 30^{\text {th }}, 70^{\text {th }}$, and $95^{\text {th }}$ percentiles.

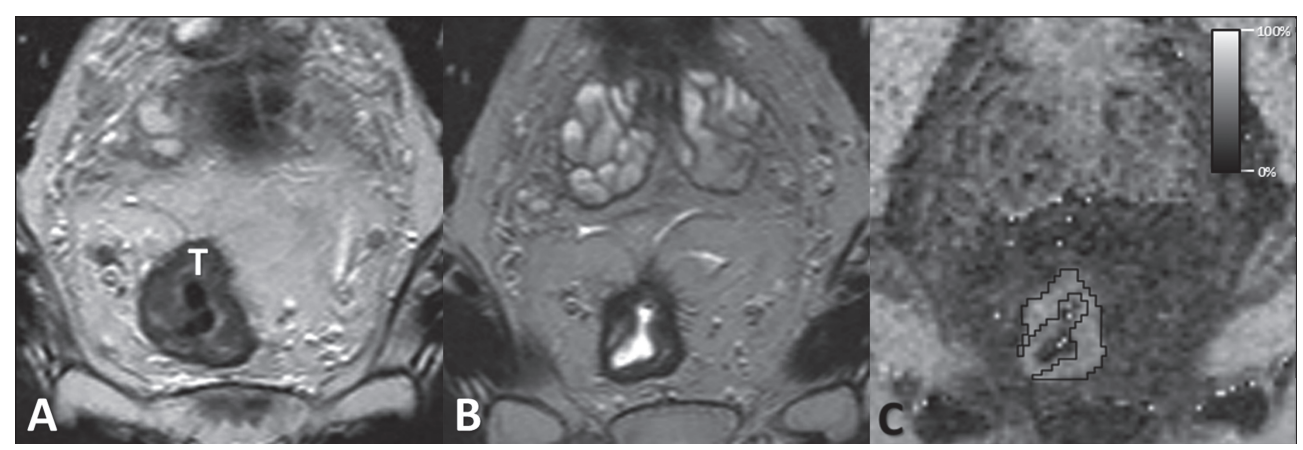

Figure 1.

(A) Axial T2-weigthed MRI at primary staging with ' $T$ ' indicating the primary tumor. (B) Axial restaging T2weighted MRI performed 8 weeks after chemoradiation, (C) corresponding axial MTR map showing the delineation of the tumor remnant.

\section{Standard of Reference}

Histopathological assessment of the surgical resection specimens served as the primary standard of reference. Histopathological assessment was performed according to the method as described by Quirke et al. ${ }^{19}$. A dedicated GI-pathologist evaluated the tumor 
regression grade (TRG) according to the method described by Mandard ${ }^{20}$ : TRG 1 (complete regression) = absence of residual cancer and fibrosis extending to the different layers of the bowel wall, TRG 2 = rare residual cancer cells scattered throughout predominantly fibrosis, TRG 3 = increase in the number of residual cancer cells but fibrosis still predominates, TRG $4=$ residual cancer outgrowing fibrosis, and TRG $5=$ absence of any regressive changes. Patients were then dichotomized into two categories: TRG 1-2 (good response) and TRG 3-5 (poor response).

\section{Statistical analyses}

Statistical analyses were performed with Statistical Package for the Social Sciences (SPSS 20.0, Inc, Chicago, Illinois, USA). Intraclass correlation coefficients (ICC) and Bland Altman plots were calculated to determine the interobserver agreement for the MTR measurements between the two readers. Receiver Operator Characteristics (ROC) curves were computed and corresponding Area's Under the Curve (AUCS) with 95\% confidence intervals were calculated to assess the diagnostic performance of the various MTR - histogram parameters for discriminating between TRG1-2 and TRG3-5. Predictive value of parameters was assessed by logistic regression analyses, parameters were selected for multivariable analyses when $p$ was $<0.20$. For the predicted probabilities for the combined MTR histogram parameters AUCS with 95\% confidence intervals were calculated.

\section{Results}

\section{Patient and treatment characteristics}

Forty-one consecutive patients were considered for inclusion. Eleven patients were excluded for the following reasons: delayed surgery after CRT ( $n=5)$, mucinous tumor $(n=4)$, or refusal of surgery despite clinical residual tumor $(n=2)$. Inclusion process and reasons for exclusion are displayed in figure 2.In total 30 patients were included (24 male, 6 female; mean age 67.7 \pm 10.8 years). The preoperative treatment consisted of a long course chemoradiation $(28 \times 1.8$ $\mathrm{Gy}+2 \times 825 \mathrm{mg} / \mathrm{m}^{2} / \mathrm{d}$ capecitabine) in 28 patients. The remaining two patients received 5x5Gy with an interval of 8-10 weeks before surgery. The median interval between the final day of radiation and the restaging MRI was 8.9 weeks (range 6.6-24.7). Eighteen patients underwent a low anterior resection, 2 an abdominopereanal resection, and 2 transanal endoscopic microsurgery. The histological tumor regression grade in these patients was TRG $1(n=4)$, TRG $2(n=5), \operatorname{TRG} 3(n=7), \operatorname{TRG} 4(n=5)$, and TRG $5(n=1)$. Eight patients were followed for $>1$ year according to a wait-and-see policy (median disease free survival of 17.5 months, range 12-21 months). These patients were all considered TRG1. In total the dichotomized tumor regression grade was as follows: $n=17$ TRG1-2 and $n=13$ TRG3-5. 


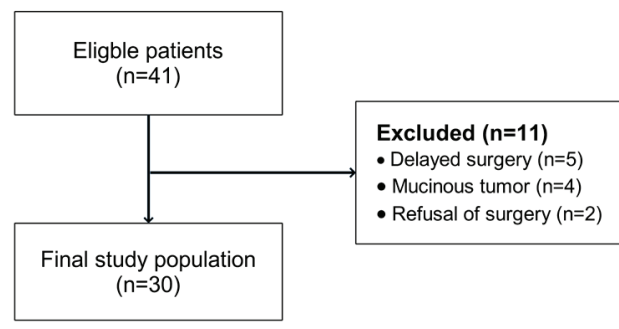

Figure 2.

CONSORT diagram of patient recruitment and reasons for exclusion.

\section{Interobserver agreement}

The intraclass correlation coefficients (ICCS) between the two readers for the mean MTR as well as the various histogram parameters are given in Table 1. ICC for the mean MTR measurements was 0.50 (moderate). The highest ICC's were obtained for the maximum MTR and the $95^{\text {th }}$ percentile with an ICC of 0.80 (good). Figure 3 displays the Bland-Altman plot for the mean MTR measurements and for the $95^{\text {th }}$ percentile. The mean bias in mean MTR between the two readers was -1.79 and limits of agreement were \pm 5.70 . For the $95^{\text {th }}$ percentile mean bias was -1.22 and limits of agreement were \pm 4.67 .

Table 1. Intraclass correlation coefficients for the mean MTR and the histogram parameters measured after chemoradiation.

\begin{tabular}{|c|c|}
\hline Parameter & Intraclass correlation \\
\hline \multicolumn{2}{|l|}{ Mean MTR } \\
\hline Mean & 0.50 \\
\hline \multicolumn{2}{|c|}{ Histogram parameters } \\
\hline Min & 0.12 \\
\hline Max & 0.80 \\
\hline Median & 0.55 \\
\hline Standard deviation & 0.53 \\
\hline Skewness & 0.29 \\
\hline Kurtosis & 0.36 \\
\hline 5th percentile & 0.29 \\
\hline 30th percentile & 0.45 \\
\hline 70th percentile & 0.64 \\
\hline 95th percentile & 0.80 \\
\hline
\end{tabular}



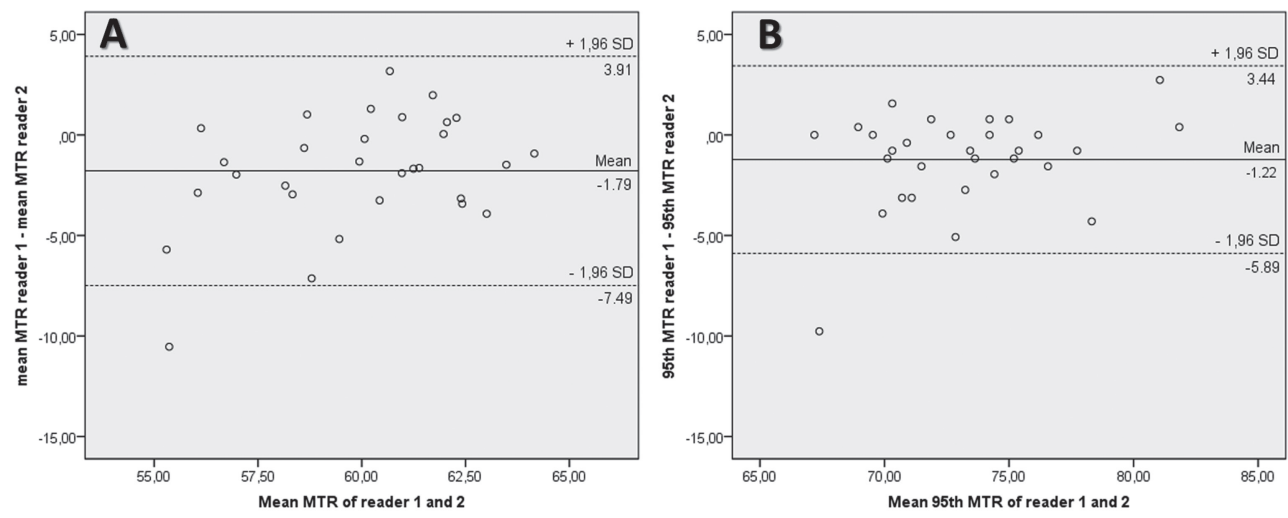

Figure 3.

Interobserver reproducibility for the mean MTR measurements (A) and $95^{\text {th }}$ percentile MTR (B). Bland-Altman plot of the averaged measurement of the two readers ( $x$-axis) plotted against the difference in measurement between the two readers (y-axis). The continuous line represents the mean absolute difference (bias) between the two readers; the dashed lines represent the $95 \%$ confidence intervals of the mean difference (limits of agreement).

\section{MTR and histogram parameters}

The mean MTR was 59.6 \pm 3.4 (TRG1-2) versus 58.4 \pm 3.1 (TRG3-5) for reader 1 ( $p=0.33$ ) and 62.1 \pm 1.9 (TRG1-2) versus $59.2 \pm 1.9$ (TRG3-5) for reader 2 ( $p<0.001$ ). Figure 4 shows the distribution in mean MTR values (averaged for the two readers) between the different tumor regression grades. The mean $95^{\text {th }}$ percentile was $73.9 \pm 4.9$ (TRG1-2) versus $70.9 \pm 1.8$ (TRG3-5) for reader 1 $(p=0.029)$ and $75.7 \pm 3.0$ (TRG1-2) versus 71.4 $\pm 2.4($ TRG3-5) for reader 2 ( $p<0.001)$. Table 2 shows the results of the receiver operator characteristics curve analyses for discriminating between TRG 1-2 and TRG 3-5 for the various histogram parameters as well as for the combined assessment of (1) Mean MTR + Skewness (2) Mean MTR $+95^{\text {th }}$ percentile, (3) Skewness $+95^{\text {th }}$ percentile, and (4) Mean MTR + Skewness $+95^{\text {th }}$ percentile. The latter combinations were derived from the logistic regression analyses.

The best single parameter to discriminate between TRG 1-2 and TRG 3-5 was the $95^{\text {th }}$ percentile for both readers (AUC 0.75 for reader 1 and 0.88 for reader 2). The various combinations of Mean MTR, Skewness and the 95 ${ }^{\text {th }}$ percentile resulted in AUCs of 0.72-0.81 for reader 1 and $0.87-0.90$ for reader 2 . This was not a significant improvement compared with the $95^{\text {th }}$ percentile as a single parameter ( $p=0.28-1.00)$. Sensitivities, specificities, positive predictive value, negative predictive value, and overall accuracies of the $95^{\text {th }}$ percentile and the various combinations are presented in table 3 . Sensitivities and specificities for diagnosing a good response ranged between $71-77 \%$ and $77-92 \%$, respectively. 
Table 2. Results from the Receiver Operator Characteristics Curve analyses for the different individual histogram parameters and various parameters combined in differentiating TRG1-2 from TRG3-5.

\begin{tabular}{|c|c|c|c|c|}
\hline \multirow{2}{*}{ Parameter } & \multicolumn{2}{|c|}{ Reader 1} & \multicolumn{2}{|c|}{ Reader 2} \\
\hline & AUC & $95 \% \mathrm{Cl}$ & AUC & $95 \% \mathrm{Cl}$ \\
\hline \multicolumn{5}{|l|}{ Mean MTR } \\
\hline Mean & 0.65 & $0.45-0.85$ & 0.87 & $0.75-1.00$ \\
\hline \multicolumn{5}{|l|}{ Histogram parameters } \\
\hline Min & 0.67 & $0.47-0.87$ & 0.72 & $0.53-0.91$ \\
\hline Max & 0.67 & $0.45-0.88$ & 0.58 & $0.37-0.80$ \\
\hline Median & 0.62 & $0.41-0.83$ & 0.86 & $0.72-0.99$ \\
\hline Standard deviation & 0.62 & $0.41-0.82$ & 0.66 & $0.46-0.85$ \\
\hline Skewness & 0.72 & $0.54-0.91$ & 0.58 & $0.37-0.78$ \\
\hline Kurtosis & 0.70 & $0.51-0.89$ & 0.65 & $0.44-0.86$ \\
\hline 5th percentile & 0.50 & $0.28-0.72$ & 0.65 & $0.45-0.85$ \\
\hline 30th percentile & 0.55 & $0.34-0.77$ & 0.81 & $0.66-0.97$ \\
\hline 70th percentile & 0.68 & $0.48-0.87$ & 0.88 & $0.76-1.00$ \\
\hline 95th percentile & 0.75 & $0.56-0.94$ & 0.88 & $0.76-1.00$ \\
\hline \multicolumn{5}{|l|}{ Combined parameters } \\
\hline Mean + skewness & 0.72 & $0.54-0.91$ & 0.87 & $0.75-1.00$ \\
\hline Mean $+95^{\text {th }}$ percentile & 0.75 & $0.56-0.94$ & 0.90 & $0.78-1.00$ \\
\hline Skewness $+95^{\text {th }}$ percentile & 0.81 & $0.65-0.97$ & 0.89 & $0.77-1.00$ \\
\hline Mean + skewness $+95^{\text {th }}$ percentile & 0.79 & $0.63-0.95$ & 0.90 & $0.78-1.00$ \\
\hline
\end{tabular}

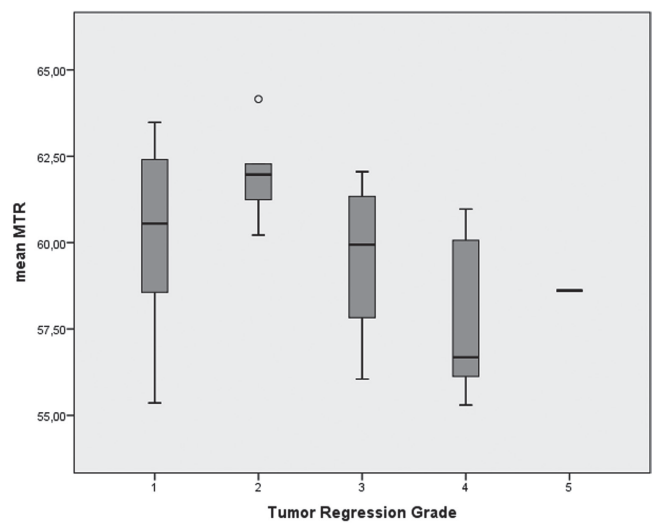

\section{Figure 4.}

Boxplot showing the distribution of mean MTR values between the different TRG subgroups. MTR values were averaged between the two readers.

Table 3: Diagnostic accuracy of MTR parameters

\begin{tabular}{|c|c|c|c|c|c|c|c|c|}
\hline Parameter & Reader & AUC & Cut-off & Sensitivity & Specificity & PPV & NPV & $\begin{array}{l}\text { Overall } \\
\text { accuracy }\end{array}$ \\
\hline \multirow{2}{*}{$95^{\text {th }}$ percentile } & Reader 1 & 0.75 & $72.9 \%$ & 70.6 & 84.6 & 68.8 & 85.1 & 76.7 \\
\hline & Reader 2 & 0.88 & $74.0 \%$ & 70.6 & 84.6 & 68.8 & 85.7 & 76.7 \\
\hline \multirow{2}{*}{$\begin{array}{l}\text { Mean }+ \\
95^{\text {th }} \text { percentile }\end{array}$} & Reader 1 & 0.75 & -0.14 & 70.6 & 92.3 & 70.6 & 92.3 & 80.0 \\
\hline & Reader 2 & 0.90 & -0.06 & 76.5 & 84.6 & 73.3 & 86.7 & 80.0 \\
\hline \multirow{2}{*}{$\begin{array}{l}\text { Skewness + } \\
95^{\text {th }} \text { percentile }\end{array}$} & Reader 1 & 0.81 & -0.20 & 76.5 & 84.6 & 73.3 & 86.7 & 80.0 \\
\hline & Reader 2 & 0.89 & -0.06 & 76.5 & 84.6 & 73.3 & 86.7 & 80.0 \\
\hline \multirow{2}{*}{$\begin{array}{l}\text { Mean + skewness+ } \\
95^{\text {th }} \text { percentile }\end{array}$} & Reader 1 & 0.79 & -0.19 & 70.6 & 76.9 & 66.7 & 80.0 & 73.3 \\
\hline & Reader 2 & 0.90 & -0.06 & 76.5 & 84.6 & 73.3 & 86.7 & 80.0 \\
\hline
\end{tabular}

$\mathrm{AUC}=$ area under the receiver operating characteristics curve, $\mathrm{PPV}=$ positive predicting value, NPV = negative predicting value. 


\section{Discussion}

The purpose of this study was to evaluate the potential value of whole-tumor MT-imaging to assess response after neoadjuvant chemoradiation for rectal cancer. We compared the mean MTR as well as various histogram parameters derived from the MTR between patients with a good response after CRT (TRG 1-2) and patients with a poor response (TRG 3-5). Results showed that MT imaging of the whole tumor volume after CRT could predict a TRG1-2 at histology with AUCS up to 0.88 (for the $95^{\text {th }}$ percentile), thereby confirming that MTR is a potentially useful help assess fibrosis.

Interestingly, the best results were obtained for the histogram parameters, in particular for the $95^{\text {th }}$ percentile which resulted in high AUCs for both readers $(0.75$ and 0.88 , respectively). The $95^{\text {th }}$ percentile represents the value above which the highest $5 \%$ of the MTR measurements are within a given ROI. In other words, the $95^{\text {th }}$ percentile represents the most fibrotic areas within the ROI. It is anticipated that the $95^{\text {th }}$ percentile will be higher in patients with a good response (with predominant fibrosis), and lower in patients with a poor response. This is indeed supported by the findings of our current study. It also confirms our secondary hypothesis that - compared to only analyzing the mean MTR - histogram data analysis is advantageous in that it gives us a better insight into the distribution pattern of MTR and therefore on the heterogeneity of the tumor area. Moreover, with a good intraclass correlation coefficient of 0.80 , the $95^{\text {th }}$ percentile appears to be more robust than mean MTR values, which showed only a moderate ICC of 0.50 . Interobserver reproducibility may thus be a limiting factor when assessing MTR. For the mean MTR measurements, the difference observed between patients with TRG1-2 and TRG3-5 was in fact smaller than the limits of agreement for the difference in MTR measurements between the two readers as derived from the Bland-Altman analysis. Although ICC was considerably better for the $95^{\text {th }}$ percentile, the mean difference between the two response groups was still smaller than the differences in measurements between the two readers. This limits its clinical utility, but could partially be explained by the current study methodology, in which ROI placement was performed directly on the MTR-maps. The MTR maps (see figure 1) lack anatomical detail, making it challenging for radiologists to interpret them. Although corresponding T2-weighted images were used for anatomical reference during the delineation process, it may be worthwhile to investigate whether it could help to draw the ROIs and copy them from another sequence, such as the standard T2-weighted images. A second potential disadvantage of quantitative imaging such as MT-imaging is that it can be a somewhat incomprehensible concept for clinicians to interpret. A simpler, qualitative approach, such as an MRI-based scoring system may be easier for clinicians to understand. The Royal Marsden research group have investigated in the MrTRG, an MRI-based scoring system of the tumor regression grade, which is constructed similar to the pathological Mandard model ${ }^{21}$. In their report, the degree of fibrosis determined at pathology was significantly associated with the mrTRG, however with moderate interobserver agreement $(0.55)^{22}$. This qualitative approach has not been 
reproduced or validated by other groups so far. For future studies, it would be interesting to compare this qualitative approach with MT-imaging for the assessment of fibrosis.

Our current study results are in line with a previous proof of principle study in 26 patients in which a single "sample" slice of the MT sequence was obtained after CRT. In this previous study, the single slice MTR map was correlated to a corresponding whole-mount histopathology section and individual areas of fibrosis, tumor, and the normal rectal wall were compared in detail with the underlying histology. This resulted in a significant difference in the mean MTR between areas of pathologically proven fibrosis and non-fibrotic areas including residual tumor ${ }^{16}$. In the current study we did not perform such a detailed whole-mount correlation with histopathology but evaluated the overall MTR of the entire tumor area (obtained from multislice MT-imaging) to discriminate between good (TRG1-2) and poor response (TRG35) to assess the value of measuring MTR from a more clinical, patient-based perspective. It confirmed that whole-tumor MTR can help differentiate between different response groups (i.e. patients with varying degrees of fibrosis). Nevertheless MTR measurements mainly reflect an estimation of the amount of fibrosis and specific information about the presence and extent of residual tumor cells is limited. In this light, it might be interesting to combine MT-imaging with other tools that specifically target detection of residual tumor, such as diffusion-weighted MR imaging to obtain a more complete understanding of the overall tumor response. Diffusion weighted imaging has shown great promise in various studies as a sensitive technique for detecting residual tumor within post-radiation fibrosis with AUCs in the range of $0.87-0.93^{13,14,23,24}$. As such, the two techniques may be of complementary value in assessing the effects of chemoradiotherapy and evaluating the various aspects of treatment response.

The MT ratios found in our study are higher than typically reported in literature. This can probably be explained by a combination of factors. First, we used on on-resonance MT prepulse, because on-resonance pulse has been suggested to result in are more prominent MT effect (resulting in higher MT ratios) compared to use of an off-resonance MT pulse ${ }^{25}$, ${ }^{26}$, which are more commonly used in current literature. Other factors that could potentially contribute to the relatively high MT ratios in our study are the use of a multislice sequence, and the dynamic acquisition of the MT sequence (simultaneous acquisition of images with and without MT pulse in one sequence). The fact that factors such as acquisition parameters can affect the MTR reveals the need for standardization and calibration of the MT-sequence. There are some limitations to our study design. First, the studied patient group was relatively small. Second, there were some small differences in the neoadjuvant treatment scheme within the study group. And finally, not in all patients histopathological confirmation was obtained. Eight patients were followed according to a wait-and-see protocol with a recurrence-free follow up period of 12-21 months, which was considered a surrogate endpoint for a complete response (TRG 1). 
In conclusion, MT-imaging is a promising tool to assess response after neoadjuvant chemoradiation in rectal cancer. In addition to measuring the mean MTR, histogram data assessment (in particular the $95^{\text {th }}$ percentile) proved beneficial and resulted in high AUCs for differentiating between patients with a good and poor response. A potential limitation of MTR is the limited interobserver agreement, which may be improved by adjusting the measurement protocol, an item that should be addressed by future studies.

\section{References}

1. Sauer R, Becker H, Hohenberger W, et al. Preoperative versus postoperative chemoradiotherapy for rectal cancer. N Engl J Med 2004; 351(17): 1731-40.

2. Coco C, Rizzo G, Mattana C, et al. Transanal endoscopic microsurgery after neoadjuvant radiochemotherapy for locally advanced extraperitoneal rectal cancer: short-term morbidity and functional outcome. Surgical endoscopy 2013; 27(8): 2860-7.

3. Habr-Gama A, Perez RO, Nadalin W, et al. Operative versus nonoperative treatment for stage 0 distal rectal cancer following chemoradiation therapy: long-term results. Annals of surgery 2004; 240(4): 711-7; discussion 7-8.

4. Maas M, Beets-Tan RG, Lambregts DM, et al. Wait-and-see policy for clinical complete responders after chemoradiation for rectal cancer. J Clin Oncol 2011; 29(35): 4633-40.

5. Smith JD, Ruby JA, Goodman KA, et al. Nonoperative management of rectal cancer with complete clinical response after neoadjuvant therapy. Annals of surgery 2012; 256(6): 965-72.

6. Habr-Gama A, Perez RO, Wynn G, Marks J, Kessler H, Gama-Rodrigues J. Complete clinical response after neoadjuvant chemoradiation therapy for distal rectal cancer: characterization of clinical and endoscopic findings for standardization. Dis Colon Rectum 2010; 53(12): 1692-8.

7. Vliegen RF, Beets GL, Lammering G, et al. Mesorectal fascia invasion after neoadjuvant chemotherapy and radiation therapy for locally advanced rectal cancer: accuracy of MR imaging for prediction. Radiology 2008; 246(2): 454-62.

8. Beets-Tan RG, Lambregts DM, Maas M, et al. Magnetic resonance imaging for the clinical management of rectal cancer patients: recommendations from the 2012 European Society of Gastrointestinal and Abdominal Radiology (ESGAR) consensus meeting. Eur Radiol 2013; 23(9): 2522-31.

9. Barbaro B, Vitale R, Leccisotti L, et al. Restaging locally advanced rectal cancer with MR imaging after chemoradiation therapy. Radiographics: a review publication of the Radiological Society of North America, Inc 2010; 30(3): 699-716.

10. van der Paardt MP, Zagers MB, Beets-Tan RG, Stoker J, Bipat S. Patients Who Undergo Preoperative Chemoradiotherapy for Locally Advanced Rectal Cancer Restaged by Using Diagnostic MR Imaging: A Systematic Review and Meta-Analysis. Radiology 2013; 269(1): 101-12.

11. Kluza E, Rozeboom ED, Maas M, et al. T2 weighted signal intensity evolution may predict pathological complete response after treatment for rectal cancer. Eur Radiol 2013; 23(1): 253-61.

12. Gollub MJ, Gultekin DH, Akin O, et al. Dynamic contrast enhanced-MRI for the detection of pathological complete response to neoadjuvant chemotherapy for locally advanced rectal cancer. Eur Radio/ 2011; 22(4): 821-31. 
13. Lambregts DM, Rao SX, Sassen S, et al. MRI and Diffusion-Weighted MRI Volumetry for Identification of Complete Tumor Responders After Preoperative Chemoradiotherapy in Patients With Rectal Cancer: A Bi-institutional Validation Study. Annals of surgery 2014; Epub ahead of print.

14. Kim SH, Lee JM, Hong SH, et al. Locally advanced rectal cancer: added value of diffusion-weighted MR imaging in the evaluation of tumor response to neoadjuvant chemo- and radiation therapy. Radiology 2009; 253(1): 116-25.

15. Adler J, Swanson SD, Schmiedlin-Ren P, et al. Magnetization transfer helps detect intestinal fibrosis in an animal model of Crohn disease. Radiology 2011; 259(1): 127-35.

16. Martens MH, Lambregts DM, Papanikolaou N, et al. Magnetization transfer ratio: a potential biomarker for the assessment of postradiation fibrosis in patients with rectal cancer. Invest Radiol 2014; 49(1): 29-34.

17. Heijnen LA, Lambregts DM, Mondal D, et al. Diffusion-weighted MR imaging in primary rectal cancer staging demonstrates but does not characterise lymph nodes. Eur Radiol 2013; 23(12): 3354-60.

18. The R project for statistical computing. 2014. www.r-project.org.

19. Quirke P, Durdey P, Dixon MF, Williams NS. Local recurrence of rectal adenocarcinoma due to inadequate surgical resection. Histopathological study of lateral tumour spread and surgical excision. Lancet 1986; 2(8514): 996-9.

20. Mandard AM, Dalibard F, Mandard JC, et al. Pathologic assessment of tumor regression after preoperative chemoradiotherapy of esophageal carcinoma. Clinicopathologic correlations. Cancer 1994; 73(11): 2680-6.

21. Patel UB, Taylor F, Blomqvist L, et al. Magnetic resonance imaging-detected tumor response for locally advanced rectal cancer predicts survival outcomes: MERCURY experience. J Clin Oncol; 29(28): 3753-60.

22. Patel UB, Brown $\mathrm{G}$, Rutten $\mathrm{H}$, et al. Comparison of magnetic resonance imaging and histopathological response to chemoradiotherapy in locally advanced rectal cancer. Ann Surg Oncol 2012; 19(9): 2842-52.

23. Curvo-Semedo L, Lambregts DM, Maas M, et al. Rectal cancer: assessment of complete response to preoperative combined radiation therapy with chemotherapy--conventional MR volumetry versus diffusion-weighted MR imaging. Radiology 2011; 260(3): 734-43.

24. Ha HI, Kim AY, Yu CS, Park SH, Ha HK. Locally advanced rectal cancer: diffusion-weighted MR tumour volumetry and the apparent diffusion coefficient for evaluating complete remission after preoperative chemoradiation therapy. Eur Radiol 2013; 23(12): 3345-53.

25. Phinikaridou A, Andia ME, Saha P, Modarai B, Smith A, Botnar RM. In vivo magnetization transfer and diffusion-weighted magnetic resonance imaging detects thrombus composition in a mouse model of deep vein thrombosis. Circulation Cardiovascular imaging 2013; 6(3): 433-40.

26. Papanikolaou N, Maniatis V, Pappas J, Roussakis A, Efthimiadou R, Andreou J. Biexponential T2 relaxation time analysis of the brain: correlation with magnetization transfer ratio. Invest Radiol 2002; 37(7): 363-7. 


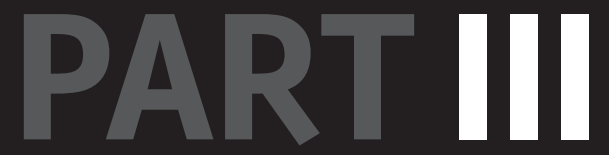

Response prediction before therapy 


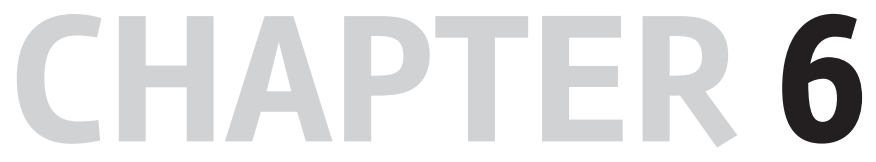

Can perfusion MRI predict response to preoperative treatment in rectal cancer?

Authors:

\author{
M.H. Martens, \\ S. Subhani, \\ L.A. Heijnen, \\ D.M.J. Lambregts, \\ J. Buijsen, \\ M. Maas, \\ R.G. Riedl, \\ C.R.L.P.N. Jeukens, \\ G.L. Beets, \\ E. Kluza, \\ R.G.H. Beets-Tan
}

Radiotherapy \& Oncology. 2015 Feb;114(2):218-23 


\section{Abstract}

\section{Background and Purpose}

Dynamic contrast-enhanced MRI (DCE-MRI) provides information on perfusion and could identify good prognostic tumors. Aim of this study was to evaluate whether DCE-MRI using a novel blood pool contrast-agent can accurately predict the response to neoadjuvant chemoradiotherapy in locally advanced rectal cancer.

\section{Material and Methods}

Thirty patients underwent DCE-MRI before and 7-10 weeks after chemoradiotherapy. Regions of interest were drawn on DCE-MRI with T2W-images as reference. DCE-MRI-based kinetic parameters (initial slope, initial peak, late slope, and AUC at 60, 90, and 120 seconds) determined pre- and post-CRT and their $\Delta$ were compared between good (TRG1-2) and poor (TRG3-5) responders. Optimal thresholds were determined and sensitivities, specificities, positive predictive values (PPV), and negative predictive values (NPV) were calculated.

\section{Results}

Pre-therapy, the late slope was able to discriminate between good and poor responders $\left(-0.05 \times 10^{-3}\right.$ vs $\left.0.62 \times 10^{-3}, p<0.001\right)$ with an AUC of 0.90 , sensitivity $92 \%$, specificity $82 \%$, PPV $80 \%$, and NPV 93\%. Other pre-CRT parameters showed no significant differences, nor any post-CRT parameters or their $\triangle$.

\section{Conclusions}

The kinetic parameter 'late slope' derived from DCE-MRI could potentially be helpful to predict before the onset of neoadjuvant chemoradiotherapy which tumors are likely going to respond. This could allow for personalized treatment-options in rectal cancer patients. 


\section{Introduction}

After neoadjuvant chemoradiotherapy (CRT) of locally advanced rectal cancer (LARC) ${ }^{1,2}$, a complete response is obtained in $8-24 \%{ }^{3-6}$. There is now a growing awareness of an organpreserving approach (local excision of the remaining scar tissue or a non-operative "wait-andsee" approach) as an alternative curative treatment for these patients ${ }^{7-9}$, given the substantial risk for morbidity and mortality associated with major resection ${ }^{10,11}$. While currently the decision for organ-preservation is made after completion of CRT, knowing upfront which patients have a high chance for a good response would be highly advantageous. Moreover, it is anticipated that the spectrum of rectal tumors manageable with organ-preservation may enlarge and selection criteria may become more liberal. However, it is not justified to irradiate all patients knowing that not all tumors will respond and still require TME, resulting in worse functional outcome compared with TME without preoperative chemoradiation. Apart from this, there may be a subgroup of patients with LARC who may have a reasonably good chance for a complete response but require intensification of preoperative treatment to increase the chance of obtaining a complete response. Therefore noninvasive tools that can predict response before the onset of CRT may allow for a more individualized treatment. Currently no reliable conventional imaging modalities exist for response prediction, because they can only provide morphological information. Dynamic Contrast-Enhanced (DCE) MRI is a functional imaging technique, mapping angiogenic microvasculature by measuring the inflow and leakage of contrast into the extracellular space. This can be expressed in signal enhancement curves from which we can quantify perfusion parameters. These parameters reflect the perfusion heterogeneity within the tumor. Therefore DCE-MRI could potentially differentiate between good and poor prognostic tumors. Few studies in literature investigated DCE-MRI for response prediction to CRT with a conventional low molecular-weight contrastagents and showed conflicting results ${ }^{12-17}$. We hypothesize that DCE-MRI based on a blood pool (e.g. high molecular-weight) contrast-agent is more accurate ${ }^{18}$.

The aim of this study was to evaluate whether perfusion parameters derived from DCE-MRI using a blood pool contrast-agent measured both before and after neoadjuvant treatment can accurately differentiate between good and poor responders in locally advanced rectal cancer. 


\section{Material and Methods}

\section{Patients}

This study was part of a prospective study on rectal cancer MRI, which was approved by the local institutional review board. Between February 2011 and March 2014, 41 consecutive patients diagnosed with LARC (cT3-4 and/or cN+) were included. Inclusion criteria consisted of (1) biopsy proven rectal cancer ( $\leq 15 \mathrm{~cm}$ of the anorectal junction), (2) LARC without distant metastases, and (3) neoadjuvant CRT. All patients underwent a primary staging MRI and a second restaging MRI 7-10 weeks after completion of CRT, both including DCE-MRI. Neoadjuvant treatment routinely consisted of 50.4Gy radiation (28x1.8Gy) combined with capecitabine $\left(825 \mathrm{mg} / \mathrm{m}^{2}\right.$ twice daily). Eleven of the 41 eligible patients were excluded for the following reasons: delayed surgery of $>3$ months after restaging MRI ( $n=7)$, artifacts on DCE-MRI hampering tumor delineation ( $n=1$ ), and mucinous tumors ( $n=3$ ) because they are known to exhibit different MR enhancing patterns compared to solid tumors.

\section{MR acquisition}

All MRI examinations were performed at 1.5T (Intera (Achieva) or Ingenia, Philips Medical Systems, Best, the Netherlands) using a phased array body coil. An intravenous bolus injection of 20 mg of butylscopolamine (Buscopan ${ }^{\circledR}$,Boehringer Ingelheim bv, Ingelheim, Germany) was administered to reduce peristaltic movement, patients did not receive bowel preparation. The standard imaging protocol consisted of T2-weighted sequences in three orthogonal planes. DCE-MRI consisted of axial dynamic T1-weighted 3D fast field echo using the following parameters: $8 \mathrm{~s}$ temporal resolution, TR/TE 7.9/4.6 ms, 30 flip angle, 11 slices, $5 \mathrm{~mm}$ slice thickness, $5 \mathrm{~mm}$ interslice distance, 220x220 mm FOV, 256x228 matrix, 6 minutes total acquisition time. After 3 series of baseline measurements (24 s), $0.12 \mathrm{ml} / \mathrm{kg}$ bodyweight of the blood pool contrast-agent gadofosveset (Ablavar ${ }^{\circledR}$, Lantheus Medical Imaging, North Billerica, Massachussets, USA) was administered at a rate of $0.70 \mathrm{ml} / \mathrm{s}$ into the brachial vein followed by a $20 \mathrm{~mL}$ saline flush using an MR compatible power injector (Spectris Solaris, MEDRAD, Warrendale, Pennsylvania, USA). The axial T2-weighted and DCE-MRI sequences were angled in identical planes, perpendicular to the tumor axis.

\section{MR analysis}

Free-hand regions of interest (ROI) were drawn by a reader, blinded for clinical and pathological data, with two years of specific expertise in rectal cancer MRI (MHM), using freely distributed medical imaging software (MRIcroN by Chris Rorden, University of South Carolina, Columbia, South Carolina, USA). The ROIs were drawn on the DCE-MRI of both the primary and post-CRT MRI. The axial T2W images were used as a reference to detect the primary tumor and the suspected residual tumor, defined as residual wall thickening with either an iso- or hypointense signal intensity at the former tumor location (figure 1). 


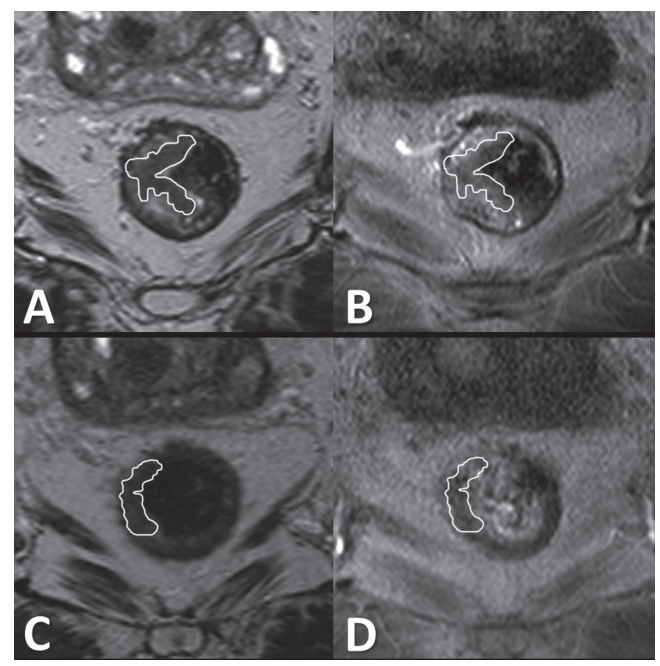

\section{Figure 1.}

Representative T2 weighted images (A \& C) and corresponding dynamic contrast enhanced MRI acquired 56 seconds after administration of gadofosveset ( $B$ \& $D$ ) in a patient with locally advanced rectal cancer. The upper panel represents the MRI of the primary tumor at initial diagnosis (A \& B) and the lower panel depicts the same level after neo-adjuvant chemoradiation ( $C \& D$ ). Regions of interest of the primary tumor and the residual tissue after chemoradiation are delineated in white.

DCE-MRI analysis was performed on a voxel-by-voxel basis using Matlab (Matlab 2007, MathWorks, Natick, Massachussetts, USA). Semiquantitative data analysis was chosen in view of the complex pharmacokinetic properties of the protein-binding contrast-agent, which affects the conversion of signal enhancement to gadofosveset concentration and applicability of the current pharmacokinetic methods. For each voxel, a relative enhancement-time curve was obtained according to the equation: $S_{r e l}(t)=\left(S(t)-S_{0}\right) / S_{0}$, where $S(t)$ represents the signal intensity at a time $(t)$ and $S_{0}$ the baseline signal intensity, i.e. the average of the three precontrast scans. Six semiquantitative kinetic parameters were calculated: (1) Initial slope, based on the initial 3 upslope data points of the enhancement-time curve. (2) Initial peak, represents the magnitude of enhancement at the end of the initial slope. (3) Areas under the first 60, 90, and 120 seconds of the enhancement curve $\left(A \cup C_{60}, A \cup C_{90}\right.$, and $A \cup C_{120}$ ), the integrals of the respective data. (4) Late slope, based on the late phase of the enhancementtime curve, i.e. from the initial peak until the end of acquisition (figure 2). The aforementioned parameters are dimensionless since they were calculated from relative enhancement curves.

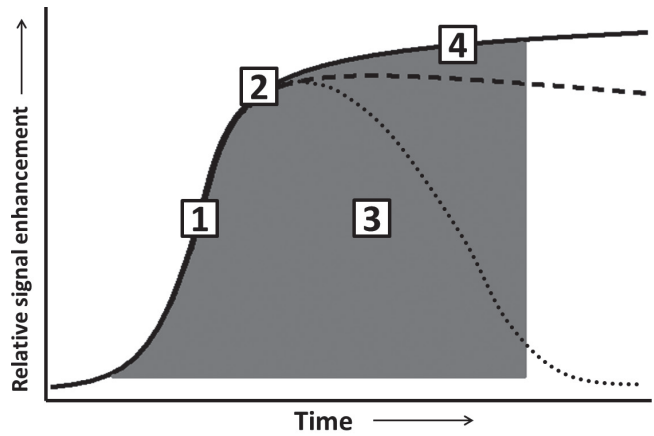

Figure 2.

Schematic representation of the contrast enhancement-time curve obtained from the dynamic contrast enhanced MRI using blood pool contrast-agent gadofosveset. (1) Initial slope, (2) initial peak, (3) area under the enhancement curve, and (4) late slope. The dashed line represents the different types of the late enhancement profile (increase or decrease). The dotted line represents the typical wash out phase in conventional gadolinium. 


\section{Histopathology}

Histopathological assessment of the resection specimen was performed according to the method of Quirke et al ${ }^{19}$. Tumor regression grade (TRG) was assessed by a dedicated pathologist according to Mandard 20; TRG1: Complete regression, no residual tumor cells, TRG2: Predominant fibrosis with isolated tumor cells, TRG3: More residual tumor cells but fibrosis still dominant, TRG4: Residual tumor outgrowing fibrosis, TRG5: no regression. Therapeutic response was stratified into two groups: Good responders were defined as TRG 1-2 and poor responders were defined as TRG $3-5^{21}$.

\section{Statistical analyses}

All statistical analyses were performed with Statistical Package for the Social Sciences (SPSS 20.0, Inc, Chicago, Illinois, USA). Normally distributed kinetic parameters were compared between good and poor responders using an independent samples T-test. The nonparametric Mann-Whitney $U$ test was performed for non-normally distributed data. P-values $<0.05$ were considered statistically significant. Multivariate analysis was not performed due to the exploratory nature of this study with a limited number of patients. For parameters that were significantly different between the response groups, receiver operating characteristic (ROC) curves were obtained and the area under the ROC curve (AUC) was calculated. The optimal cut-off value was determined as the point nearest to the upper left corner of the ROCcurve and corresponding sensitivity, specificity, positive predictive value (PPV), and negative predictive value (NPV) including 95\% confidence intervals were calculated.

\section{Results}

In total 30 patients were included, the mean age was 66 years (SD \pm 10.1 ). Table 1 displays the patient, treatment, and tumor characteristics. Four patients had a complete response on MRI (including diffusion-weighted MRI) and endoscopy and did not undergo surgery but were subjected to a stringent follow-up consisting of MRI and endoscopy. These patients showed sustained complete response for 12, 13, 13, and 32 months, respectively, and were categorized as good responders. All together 13 patients were considered good responders (TRG 1-2) and 17 patients as poor responders (TRG 3-5).

The pre-CRT mean of the late slope for good responders $\left(-0.05 \times 10^{-3} \pm 0.34 \times 10^{-3}\right)$ was significantly lower compared to that for poor responders $\left(0.62 \times 10^{-3} \pm 0.51 \times 10^{-3}, p<0.001\right)$. The pre-CRT initial slope, initial peak, $A \cup C_{60}, A \cup C_{90}$, and $A \cup C_{120}$ were not significantly different between good and poor responders (Table 2A). Figure 3 illustrates representative relative enhancement-time curves of a good and poor responder.

The means of the post-CRT initial slope, initial peak, $A \cup C_{60}, A \cup C_{90}$, and $A \cup C_{120}$, and late slope were not significantly different between good and poor responders (table 2B). The percentage of change $(\Delta)$ of the parameters was higher for good responders, but not 
Table 1. Patient-, treatment- and tumor-characteristics

\begin{tabular}{|c|c|c|c|c|}
\hline \multicolumn{2}{|c|}{ Characteristic } & $\begin{array}{l}\text { All patients } \\
(n=30)\end{array}$ & $\begin{array}{l}\text { Good responders } \\
(n=13)\end{array}$ & $\begin{array}{l}\text { Poor responders } \\
(n=17)\end{array}$ \\
\hline \multirow{2}{*}{ Sex } & Male & $23(77 \%)$ & $11(85 \%)$ & $12(71 \%)$ \\
\hline & Female & $7(23 \%)$ & $2(15 \%)$ & $5(29 \%)$ \\
\hline \multirow{3}{*}{$\begin{array}{l}\text { Primary } \\
\text { T-stage }\end{array}$} & сT2 & $2(7 \%)$ & $2(15 \%)$ & 0 \\
\hline & cT3 & $27(90 \%)$ & $11(85 \%)$ & $16(94 \%)$ \\
\hline & CT4 & $1(3 \%)$ & 0 & $1(6 \%)$ \\
\hline \multirow{3}{*}{$\begin{array}{l}\text { Primary } \\
\text { N-stage }\end{array}$} & $\mathrm{cNO}$ & $2(7 \%)$ & $1(8 \%)$ & $1(6 \%)$ \\
\hline & $\mathrm{cN} 1$ & $3(10 \%)$ & $2(15 \%)$ & $1(6 \%)$ \\
\hline & $\mathrm{cN} 2$ & $25(83 \%)$ & $10(77 \%)$ & $15(88 \%)$ \\
\hline \multirow{4}{*}{$\begin{array}{l}\text { Type of } \\
\text { surgery }\end{array}$} & Low anterior resection & $22(73 \%)$ & $7(54 \%)$ & $15(88 \%)$ \\
\hline & Abdominoperineal resection & $3(10 \%)$ & $2(15 \%)$ & $1(6 \%)$ \\
\hline & Extended resection & $1(3 \%)$ & & $1(6 \%)$ \\
\hline & No surgery & $4(13 \%)$ & $4(31 \%)$ & 0 \\
\hline \multirow{6}{*}{$\begin{array}{l}\text { ypT } \\
\text { category }\end{array}$} & урт0 & $2(7 \%)$ & $2(15 \%)$ & 0 \\
\hline & урT1 & $2(7 \%)$ & $2(15 \%)$ & 0 \\
\hline & урт2 & $5(17 \%)$ & $2(15 \%)$ & $3(18 \%)$ \\
\hline & урт3 & $16(53 \%)$ & $3(23 \%)$ & $13(77 \%)$ \\
\hline & урT4 & $1(3 \%)$ & 0 & $1(6 \%)$ \\
\hline & ypTx & $4^{\wedge}(13 \%)$ & $4^{\wedge}(31 \%)$ & 0 \\
\hline \multirow{4}{*}{$\begin{array}{l}\text { ypN } \\
\text { catergory }\end{array}$} & ypNo & $16(53 \%)$ & $8(62 \%)$ & $8(47 \%)$ \\
\hline & ypN1 & $7(23 \%)$ & $1(8 \%)$ & $6(35 \%)$ \\
\hline & ypN2 & $3(10 \%)$ & & $3(18 \%)$ \\
\hline & ypNx & $4(13 \%)$ & $4^{\wedge}(31 \%)$ & n.a. \\
\hline \multirow{6}{*}{$\mathrm{TRG}^{*}$} & 1 & $2(7 \%)$ & $2(15 \%)$ & n.a. \\
\hline & 2 & $7(23 \%)$ & $7(54 \%)$ & n.a. \\
\hline & 3 & $12(40 \%)$ & n.a. & $12(71 \%)$ \\
\hline & 4 & $4(13 \%)$ & n.a. & $4(24 \%)$ \\
\hline & 5 & $1(3 \%)$ & n.a. & $1(6 \%)$ \\
\hline & unknown & $4^{\wedge}(13 \%)$ & $4^{\wedge}(31 \%)$ & n.a. \\
\hline
\end{tabular}

* Tumor regression grade according to Mandard, ^ Consisting of the 4 non-operated patients, n.a. = not applicable

statistically significant. A trend towards significance was seen for the initial slope (35.6\% vs 23.1\%, $p=0.063$ ), initial peak (31.4\% vs 20.0\%, $p=0.084$ ), and $\mathrm{AUC}_{90}$ (35.6\% vs 23.9\%, $\left.p=0.079\right)$. The area under the ROC curve for predicting a good response based on the pre-CRT late slope was 0.90 (95\% Cl 0.78-1.00). The optimal cut-off was determined at $0.258 \times 10^{-3}$ and resulted in a sensitivity of $92 \%$ (12/13; 95\% Cl 62-100\%), specificity of 82\% (14/17; 95\% Cl 56-95\%), PPV of $80 \%$ (12/15; 95\% Cl 51-95\%), and NPV of 93\% (14/15; 95\% Cl 66-100\%). 
Table 2:

\begin{tabular}{|c|c|c|c|}
\hline Kinetic parameter & Good responders $(n=13)$ & Poor responders $(n=17)$ & p-value \\
\hline \multicolumn{4}{|c|}{ Before neo-adjuvant chemoradiotherapy } \\
\hline Initial slope & $0.039( \pm 0.006)$ & $0.037( \pm 0.012)$ & 0.570 \\
\hline Initial peak & $1.76( \pm 0.28)$ & $1.75( \pm 0.44)$ & 0.965 \\
\hline $\mathrm{AUC}_{60}{ }^{*}$ & $96( \pm 16)$ & $92( \pm 22)$ & 0.547 \\
\hline $\mathrm{AUC}_{90}{ }^{*}$ & $150( \pm 24)$ & $151( \pm 39)$ & 0.928 \\
\hline $\mathrm{AUC}_{120^{*}}$ & $204( \pm 32)$ & $207( \pm 51)$ & 0.813 \\
\hline Late slope & $-0.05 \times 10^{-3}\left( \pm 0.34 \times 10^{-3}\right)$ & $0.62 \times 10^{-3}\left( \pm 0.51 \times 10^{-3}\right)$ & $<0.001$ \\
\hline \multicolumn{4}{|c|}{ After neo-adjuvant chemoradiotherapy } \\
\hline Initial slope & $0.025( \pm 0.005)$ & $0.027( \pm 0.007)$ & 0.265 \\
\hline Initial peak & $1.19( \pm 0.26)$ & $1.35( \pm 0.24)$ & 0.085 \\
\hline $\mathrm{AUC}_{60}{ }^{*}$ & $61( \pm 13)$ & $68( \pm 15)$ & 0.201 \\
\hline $\mathrm{AUC}_{90}{ }^{\star}$ & $101( \pm 23)$ & $114( \pm 22)$ & 0.118 \\
\hline $\mathrm{AUC}_{120^{*}}$ & $144( \pm 33)$ & $162( \pm 32)$ & 0.114 \\
\hline Late slope & $1.94 \times 10^{-3}\left( \pm 1.13 \times 10^{-3}\right)$ & $1.75 \times 10^{-3}\left( \pm 0.84 \times 10^{-3}\right)$ & 0.593 \\
\hline \multicolumn{4}{|c|}{ Percentage of change after neo-adjuvant chemoradiotherapy } \\
\hline Initial slope & $35.6 \%( \pm 14.9)$ & $23.1 \%( \pm 19.3)$ & 0.063 \\
\hline Initial peak & $31.4 \%( \pm 17.0)$ & $20.0 \%( \pm 17.4)$ & 0.084 \\
\hline $\mathrm{AUC}_{60}{ }^{*}$ & $35.6 \%( \pm 16.5)$ & $23.9 \%( \pm 18.1)$ & 0.079 \\
\hline $\mathrm{AUC}_{90}{ }^{*}$ & $31.8 \%( \pm 17.0)$ & $21.4 \%( \pm 18.0)$ & 0.120 \\
\hline $\mathrm{AUC}_{120^{*}}$ & $28.5 \%( \pm 17.8)$ & $19.1 \%( \pm 18.3)$ & 0.169 \\
\hline Late slope & $75 \%( \pm 241)$ & $-4.5 \%( \pm 13.0)$ & 0.300 \\
\hline
\end{tabular}

Comparison between good and poor responders with respect to the mean of the kinetic parameters before neoadjuvant chemoradiation $(A)$, after neoadjuvant treatment $(B)$, and the percentage of change $(\Delta)$ after neoadjuvant treatment (C). Data represent the mean \pm standard deviation (SD).

${ }^{*} A \cup C_{60}, A C_{90}$, and $A \cup C_{120}=$ Areas under the first 60,90 , and 120 seconds of the enhancement curve 


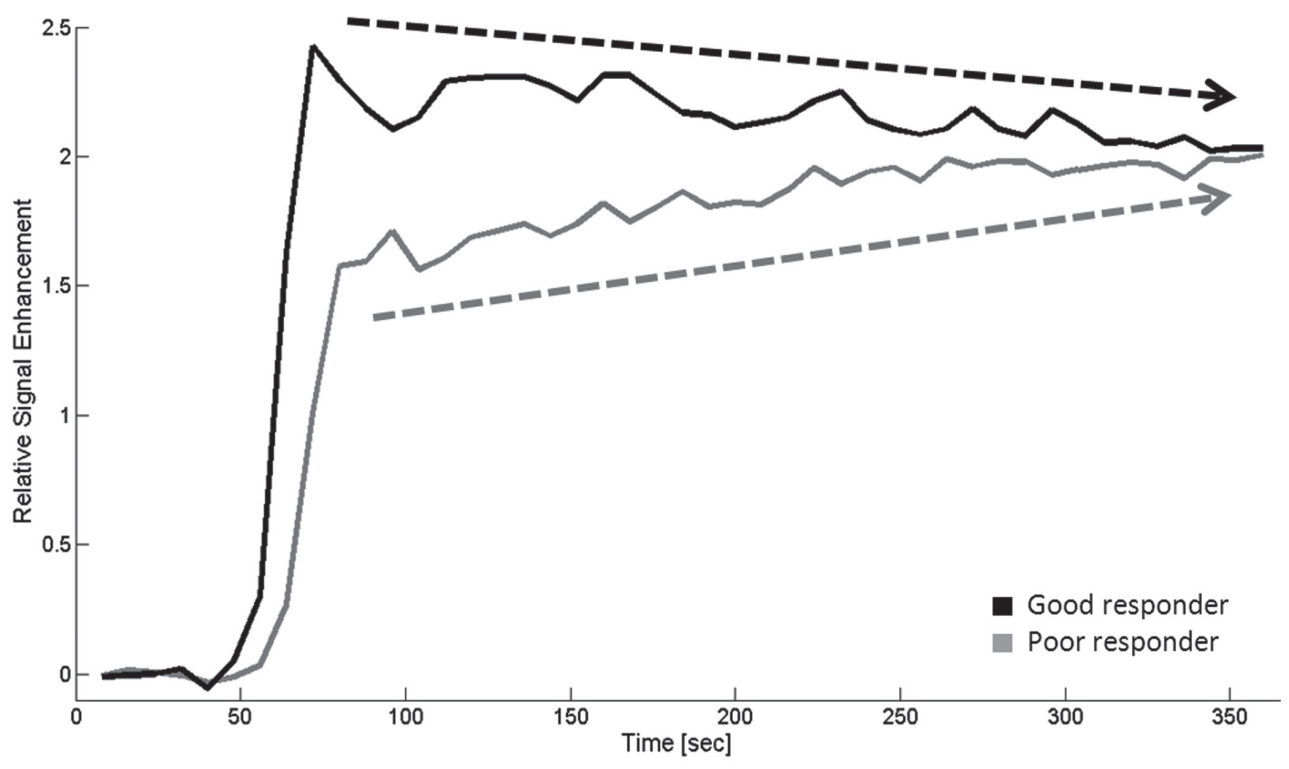

Figure 3.

Representative relative enhancement-time curves obtained for the primary tumor before neoadjuvant chemoradiation of a good responder (black line) and a poor responder (grey line). The dashed arrows indicate the late slope.

\section{Discussion}

The aim of this study was to evaluate whether kinetic parameters derived from DCE-MRI using a blood pool contrast-agent and measured before and after CRT, can accurately predict which patients will achieve a good (TRG1-2) or poor response (TRG3-5) to CRT in rectal cancer. We found that the kinetic parameter 'late slope' of the DCE-MRI enhancement curve could accurately predict response pre-CRT with an AUC 0.90. Kinetic parameters measured after CRT and their $\triangle$ post-CRT did not show significant differences between good and poor responders. DCE-MRI before onset of CRT seems promising to identify patients likely to respond very well to CRT.

Currently, no reliable imaging tools exist to accurately predict response to CRT in patients with locally advanced rectal cancer. Pretreatment prediction of response using the quantitative biomarker "apparent diffusion coefficient" (ADC) from diffusion-MRI, has been the focus of research in several studies, showing no clear value and a considerable variation in the mean ADC-values between good and poor responders ${ }^{15,22-30}$. DCE-MRI has only been sparsely investigated for response prediction with conventional gadolinium contrast agents and the results of published studies are difficult to compare because some used semiquantitative and others quantitative analysis and each focused on different parameters. For example the groups of Oberholzer ${ }^{31}$, George ${ }^{32}$, Lim ${ }^{33}$, and Intven ${ }^{34}$ focused on the quantitative 
analysis, all found higher pretreatment values for downstaged tumors compared with nondownstaged. In contrast, Devries et al., who focused on the Perfusion Index (maximum slope of concentration-time curve normalized by maximum arterial input function) found a significantly lower perfusion index in ypT0-2 tumors compared with ypT3-4 tumors 12, 14, 17 . Additionally, Kim et al. reported no significant difference in pre-therapy perfusion between downstaged and non-downstaged tumors ${ }^{16}$. The value of DCE-MRI for response prediction is therefore not clear, nor is it clear whether DCE-MRI should be post-processed in a quantitative or semiquantitative way.

We found the semiquantitative kinetic parameter 'late slope', which we calculated preCRT based on the relative enhancement-time curve, to be highly promising in predicting patients who were going to respond well to CRT (AUC 0.90, sensitivity 92\%, and specificity $82 \%)$. The pretreatment mean value of this perfusion parameter was significantly lower for good compared to poor responders $\left(-0.05 \times 10^{-3} \mathrm{vs} 0.62 \times 10^{-3}, p<0.001\right)$. To our knowledge only one paper reported on a quantitative late enhancement parameter $\left(k_{e p}\right)$ using gadolinium, and the results were negative ${ }^{16}$. With gadolinium an obvious wash-out is observed, as a result of rapid contrast clearance. However, our large molecular agent is an albumin-bound gadofosveset complex, that is formed in the blood stream during the first 30 seconds after injection and behaves as a long-circulating blood pool contrast that does not generate a typical wash-out phase (figure 2). In normal tissues, the enhancement reaches a steady state, whereas in non-irradiated tumors we found either increasing (poor response) or slightly decreasing (good response) trend (figure 3). The persistent increasing enhancement has been attributed to the steady ongoing extravasation of a large contrast-agent through the leaky tumor angiogenic vasculature ${ }^{35}$. These results support the theory that tumor angiogenic activity has a negative impact on therapy outcome ${ }^{36,37}$. We hypothesize that the intrinsic tumor aggressiveness may counteract the therapeutic effects of the chemoradiotherapy. The advantage of the large molecular gadofosveset is that it requires a lower dose to generate the same contrast compared to gadolinium, which is important for reduction of the risk of nephrogenic systemic fibrosis ${ }^{38}$.

Second, we used a 3D DCE-MRI sequence, while others used a 2D imaging sequence. It is known that 3D acquisition of perfusion MR data at satisfying temporal and spatial resolution better represent the tumor heterogeneous state of perfusion than a 2D acquisition. With the 3D method, the information is given on the entire tumor while with the 2D method this information is only of 1 or 2 slices of the tumor. In other words, a 3D perfusion MRI could hypothetically better depict subtle differences in tumor heterogeneity between good and poor prognostic tumors, allowing a more accurate prediction of the differences in its response to treatment.

The 'late slope' parameter calculated from the post-CRT DCE curves in our study did not prove efficient for the assessment of response, nor its $\triangle$ post-CRT. Although a large increase post-CRT was observed for good responders, while it remained relatively constant in poor responders, this was not significantly different (table $2 \mathrm{C}$ ). Radiation treatment is associated 
with vascular hyperpermeability ${ }^{39,40}$ and we hypothesize that as a consequence of these therapeutic effects, the 'late slope' lost its diagnostic value.

Predicting tumor response to CRT before the onset of treatment is clinically relevant since there is a paradigm shift in treatment towards organ-preservation for patients with clinical (near) complete response after CRT. Currently it is not well understood why some patients show a dramatic response while others do not. The ability to predict which patients are likely to have a (near) complete response to CRT would allow for more rational, cost effective, and personalized treatment choices. Patients who will normally undergo major surgery without neoadjuvant treatment, but are predicted to show a very good response to neoadjuvant chemoradiation, could then be considered for an organ-preservation. Patients with more advanced rectal cancer that are likely going to respond well to CRT, could be offered an intensified neoadjuvant treatment in order to increase the chance of obtaining a better response. The best prediction models will eventually include a variety of predictive factors from clinical, imaging, molecular, and other sources ${ }^{41}$.

There are some limitations to this study. First, this study was performed with a relative small sample size. Further research with a larger patient cohort is needed to confirm these results and to validate the threshold determined in the current study. Second, the patients in the present study were all locally advanced tumors, and it remains to be determined whether this predictive effect also applies to smaller tumors. Thirdly, not all patients underwent surgery after neoadjuvant treatment, because of suspected clinical complete response based on (DWI)MRI and endoscopy and stratification into an organ-preserving treatment. All four patients remained free of disease for at least 12 months.

In conclusion, we have shown that the kinetic parameter 'late slope' based on DCE-MRI with a blood pool contrast-agent could potentially be helpful to predict before the onset of treatment which patients with locally advanced rectal cancer are likely going to respond to neoadjuvant chemoradiotherapy. This would allow for a more personalized treatment stratification.

\section{References}

1. Glimelius B, Oliveira J. Rectal cancer: ESMO clinical recommendations for diagnosis, treatment and follow-up. Ann Oncol 2009; 20 Suppl 4: 54-6.

2. Engelen SM, Maas M, Lahaye MJ, et al. Modern multidisciplinary treatment of rectal cancer based on staging with magnetic resonance imaging leads to excellent local control, but distant control remains a challenge. European journal of cancer 2013; 49(10): 2311-20.

3. Maas M, Nelemans PJ, Valentini V, et al. Long-term outcome in patients with a pathological complete response after chemoradiation for rectal cancer: a pooled analysis of individual patient data. The lancet oncology 2010; 11(9): 835-44. 
4. Dhadda AS, Dickinson P, Zaitoun AM, Gandhi N, Bessell EM. Prognostic importance of Mandard tumour regression grade following pre-operative chemo/radiotherapy for locally advanced rectal cancer. European journal of cancer 2011; 47(8): 1138-45.

5. Burbach JP, den Harder AM, Intven M, van Vulpen M, Verkooijen HM, Reerink O. Impact of radiotherapy boost on pathological complete response in patients with locally advanced rectal cancer: a systematic review and meta-analysis. Radiother Oncol 2014; 113(1): 1-9.

6. Swellengrebel HA, Bosch SL, Cats A, et al. Tumour regression grading after chemoradiotherapy for locally advanced rectal cancer: a near pathologic complete response does not translate into good clinical outcome. Radiother Oncol 2014; 112(1): 44-51.

7. Habr-Gama A, Perez RO, Proscurshim I, et al. Patterns of failure and survival for nonoperative treatment of stage c0 distal rectal cancer following neoadjuvant chemoradiation therapy. Journal of gastrointestinal surgery: official journal of the Society for Surgery of the Alimentary Tract 2006; 10(10): 1319-28; discussion 28-9.

8. Maas M, Beets-Tan RG, Lambregts DM, et al. Wait-and-see policy for clinical complete responders after chemoradiation for rectal cancer. J Clin Oncol 2011; 29(35): 4633-40.

9. Garcia-Aguilar J, Shi Q, Thomas CR, Jr., et al. A phase II trial of neoadjuvant chemoradiation and local excision for T2NO rectal cancer: preliminary results of the ACOSOG Z6041 trial. Ann Surg Oncol 2012; 19(2): 384-91.

10. Marijnen CA, Kapiteijn E, van de Velde CJ, et al. Acute side effects and complications after shortterm preoperative radiotherapy combined with total mesorectal excision in primary rectal cancer: report of a multicenter randomized trial. J Clin Oncol 2002; 20(3): 817-25.

11. Loos M, Quentmeier P, Schuster T, et al. Effect of preoperative radio (chemo)therapy on long-term functional outcome in rectal cancer patients: a systematic review and meta-analysis. Ann Surg Oncol 2013; 20(6): 1816-28.

12. Devries AF, Griebel J, Kremser C, et al. Tumor microcirculation evaluated by dynamic magnetic resonance imaging predicts therapy outcome for primary rectal carcinoma. Cancer Res 2001; 61(6): 2513-6.

13. Gollub MJ, Gultekin DH, Akin O, et al. Dynamic contrast enhanced-MRI for the detection of pathological complete response to neoadjuvant chemotherapy for locally advanced rectal cancer. Eur Radio/ 2011; 22(4): 821-31.

14. Kremser C, Trieb T, Rudisch A, Judmaier W, de Vries A. Dynamic T(1) mapping predicts outcome of chemoradiation therapy in primary rectal carcinoma: sequence implementation and data analysis. J Magn Reson Imaging 2007; 26(3): 662-71.

15. Intven M, Reerink O, Philippens ME. Diffusion-weighted MRI in locally advanced rectal cancer : pathological response prediction after neo-adjuvant radiochemotherapy. Strahlentherapie und Onkologie: Organ der Deutschen Rontgengesellschaft [et al] 2013; 189(2): 117-22.

16. Kim SH, Lee JM, Gupta SN, Han JK, Choi BI. Dynamic contrast-enhanced MRI to evaluate the therapeutic response to neoadjuvant chemoradiation therapy in locally advanced rectal cancer. J Magn Reson Imaging 2014; 40(3): 730-7.

17. DeVries AF, Piringer G, Kremser C, et al. Pretreatment evaluation of microcirculation by dynamic contrast-enhanced magnetic resonance imaging predicts survival in primary rectal cancer patients. International journal of radiation oncology, biology, physics 2014; 90(5): 1161-7. 
18. de Lussanet QG, Langereis S, Beets-Tan RG, et al. Dynamic contrast-enhanced MR imaging kinetic parameters and molecular weight of dendritic contrast agents in tumor angiogenesis in mice. Radiology 2005; 235(1): 65-72.

19. Quirke P, Durdey P, Dixon MF, Williams NS. Local recurrence of rectal adenocarcinoma due to inadequate surgical resection. Histopathological study of lateral tumour spread and surgical excision. Lancet 1986; 2(8514): 996-9.

20. Mandard AM, Dalibard F, Mandard JC, et al. Pathologic assessment of tumor regression after preoperative chemoradiotherapy of esophageal carcinoma. Clinicopathologic correlations. Cancer 1994; 73(11): 2680-6.

21. Vecchio FM, Valentini V, Minsky BD, et al. The relationship of pathologic tumor regression grade (TRG) and outcomes after preoperative therapy in rectal cancer. International journal of radiation oncology, biology, physics 2005; 62(3): 752-60.

22. DeVries AF, Kremser C, Hein PA, et al. Tumor microcirculation and diffusion predict therapy outcome for primary rectal carcinoma. International journal of radiation oncology, biology, physics 2003; 56(4): 958-65.

23. Ippolito D, Monguzzi L, Guerra L, et al. Response to neoadjuvant therapy in locally advanced rectal cancer: assessment with diffusion-weighted MR imaging and 18FDG PET/CT. Abdom Imaging 2012; 37(6): 1032-40.

24. Kim YC, Lim JS, Keum KC, et al. Comparison of diffusion-weighted MRI and MR volumetry in the evaluation of early treatment outcomes after preoperative chemoradiotherapy for locally advanced rectal cancer. J Magn Reson Imaging 2011; 34(3): 570-6.

25. Lambrecht M, Vandecaveye V, De Keyzer F, et al. Value of diffusion-weighted magnetic resonance imaging for prediction and early assessment of response to neoadjuvant radiochemotherapy in rectal cancer: preliminary results. International journal of radiation oncology, biology, physics 2012; 82(2): 863-70.

26. Monguzzi L, Ippolito D, Bernasconi DP, Trattenero C, Galimberti S, Sironi S. Locally advanced rectal cancer: value of $A D C$ mapping in prediction of tumor response to radiochemotherapy. European journal of radiology 2013; 82(2): 234-40.

27. Musio D, De Felice F, Magnante AL, et al. Diffusion-Weighted Magnetic Resonance Application in Response Prediction before, during, and after Neoadjuvant Radiochemotherapy in Primary Rectal Cancer Carcinoma. BioMed research international 2013; 2013: 740195.

28. Jung SH, Heo SH, Kim JW, et al. Predicting response to neoadjuvant chemoradiation therapy in locally advanced rectal cancer: diffusion-weighted 3 Tesla MR imaging. J Magn Reson Imaging 2012; 35(1): 110-6.

29. Sun YS, Zhang XP, Tang L, et al. Locally advanced rectal carcinoma treated with preoperative chemotherapy and radiation therapy: preliminary analysis of diffusion-weighted MR imaging for early detection of tumor histopathologic downstaging. Radiology 2010; 254(1): 170-8.

30. Barbaro $B$, Vitale $R$, Valentini $V$, et al. Diffusion-weighted magnetic resonance imaging in monitoring rectal cancer response to neoadjuvant chemoradiotherapy. International journal of radiation oncology, biology, physics 2012; 83(2): 594-9.

31. Oberholzer K, Menig M, Pohlmann A, et al. Rectal cancer: assessment of response to neoadjuvant chemoradiation by dynamic contrast-enhanced MRI. J Magn Reson Imaging 2013; 38(1): 119-26. 
32. George ML, Dzik-Jurasz AS, Padhani AR, et al. Non-invasive methods of assessing angiogenesis and their value in predicting response to treatment in colorectal cancer. Br J Surg 2001; 88(12): 1628-36.

33. Lim JS, Kim D, Baek SE, et al. Perfusion MRI for the prediction of treatment response after preoperative chemoradiotherapy in locally advanced rectal cancer. Eur Radiol 2012; 22(8): 1693700.

34. Intven M, Reerink O, Philippens ME. Dynamic contrast enhanced MR imaging for rectal cancer response assessment after neo-adjuvant chemoradiation. J Magn Reson Imaging 2015; 41(6): 1646-53.

35. Barrett T, Kobayashi H, Brechbiel M, Choyke PL. Macromolecular MRI contrast agents for imaging tumor angiogenesis. European journal of radiology 2006; 60(3): 353-66.

36. Sahani DV, Kalva SP, Hamberg LM, et al. Assessing tumor perfusion and treatment response in rectal cancer with multisection CT: initial observations. Radiology 2005; 234(3): 785-92.

37. Johansen R, Jensen LR, Rydland J, et al. Predicting survival and early clinical response to primary chemotherapy for patients with locally advanced breast cancer using DCE-MRI. J Magn Reson Imaging 2009; 29(6): 1300-7.

38. Canga A, Kislikova M, Martinez-Galvez M, et al. Renal function, nephrogenic systemic fibrosis and other adverse reactions associated with gadolinium-based contrast media. Nefrologia: publicacion oficial de la Sociedad Espanola Nefrologia 2014; 34(4): 428-38.

39. Mayr NA, Yuh WT, Arnholt JC, et al. Pixel analysis of MR perfusion imaging in predicting radiation therapy outcome in cervical cancer. J Magn Reson Imaging 2000; 12(6): 1027-33.

40. Ng QS, Goh V, Milner J, Padhani AR, Saunders MI, Hoskin PJ. Acute tumor vascular effects following fractionated radiotherapy in human lung cancer: In vivo whole tumor assessment using volumetric perfusion computed tomography. International journal of radiation oncology, biology, physics 2007; 67(2): 417-24.

41. Lambin P, van Stiphout RG, Starmans MH, et al. Predicting outcomes in radiation oncology-multifactorial decision support systems. Nature reviews Clinical oncology 2013; 10(1): 27-40. 


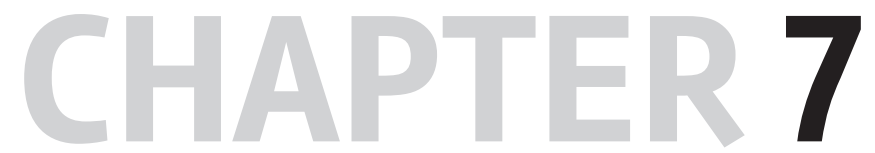

\title{
Non-invasive MR assessment of macroscopic and microscopic vascular abnormalities in the rectal tumor-surrounding mesorectum
}

\author{
Authors: \\ E. Kluza, \\ M.H. Martens, \\ J-P.J.E. Kleijnen, \\ D. Rennspiess, \\ M. Maas, \\ C.R.L.P.N. Jeukens, \\ R.G. Riedl, \\ A. zur Hausen, \\ G.L. Beets, \\ R.G.H. Beets-Tan
}

European Radiology, 2015 Aug 30 [Epub ahead of print] 


\section{Abstract \\ Objectives}

To evaluate the MRI macroscopic and microscopic parameters of mesorectal vasculature in rectal cancer patients.

\section{Methods}

13 patients with rectal adenocarcinoma underwent a dynamic contrast-enhanced MRI at 1.5T using a blood pool agent at the primary staging. Mesorectal macrovascular features, i.e. the number of vascular branches, average diameter and length, were assessed from baselinesubtracted post-contrast images by two independent readers. Mesorectal microvascular function was investigated by means of area under the enhancement-time curve (AUC). Histopathology served as reference standard of the tumor response to CRT.

\section{Results}

The average vessel branching in the mesorectum around the tumor and normal rectal wall was $8.2 \pm 3.8$ and $1.7 \pm 1.3$, respectively (reader $1 p=0.001$, reader $2 p=0.002$ ). Similarly, the tumor-surrounding mesorectum displayed circa ten-fold elevated AUC ( $p=0.01)$. Interestingly, patients with primary node involvement had two-fold higher number of macrovascular branches compared to those with healthy nodes (reader1: $p=0.005$ and reader2: $p=0.03$ ). A similar difference was observed between good and poor responders to CRT, whose tumorsurrounding mesorectum displayed $10.7 \pm 3.4$ and $5.6 \pm 1.5$ vessels, respectively (reader 1 $p=0.02$, reader $2 p=0.02$ ).

\section{Conclusions}

We showed at baseline MRI of rectal tumors a significantly enhanced macrovascular structure and microvascular function in rectal tumor-surrounding mesorectum, and the association of primary mesorectal macrovascular parameters with node involvement and therapy response. 


\section{Introduction}

Blood vasculature is an important constituent of tumor stroma and the proposed medium for cancer cell dissemination from the primary tumor to distant metastatic sites ${ }^{1-4}$. Notably, the formation of reactive vascular stroma also involves the tumor-surrounding host tissues. In these areas, the pre-existing vessels enlarge and actively engage in sprouting and branching to meet the metabolic demands of adjacent malignancy and create the invasion forefront into the neighboring tissue ${ }^{5-9}$.

In contrast to the intensively studied tumor vasculature, the vascular status of tumorsurrounding host tissue and its diagnostic significance are poorly explored. Previously, the enhanced microvascular function in peri-tumoral areas and its potential prognostic value was reported in brain cancer patients ${ }^{6,10}$. Moreover, the initial findings in brain and breast cancer suggest that the tumor microenvironment influences host vasculature also on the macroscopic level, which might be exploited for prognostic and predictive purposes ${ }^{11,12}$. The vascular assessment of tumor-surrounding host tissue might therefore provide clinically relevant information and complement the current diagnostic strategies in oncology.

In rectal cancer, the tumor extension into the mesorectal fat and mesorectal node involvement are important risk factors, which determine the patient's prognosis and treatment. Moreover, tumor invasion into the surrounding mesorectal vessels has been proposed as a relevant prognostic marker ${ }^{13-15}$. Previously, the non-invasive detection of mesorectal vasculature was based on conventional anatomical T2-weighted images, by exploiting the intrinsic negative contrast generated by deoxygenated venous blood ${ }^{14}$. However, anatomical imaging provides only limited vascular information and, thus, more sophisticated vascular imaging methods are needed to advance the knowledge on the mesorectal vascular status and its clinical relevance in rectal cancer patients. Among the clinically applicable methods, vascular imaging with an albumin-binding blood pool contrast agent, gadofosveset, appears to be especially attractive as it enables simultaneous probing of macro- and microvascular properties ${ }^{16,17}$.

Our aim was to determine both macro- and microvascular properties of the tumorsurrounding mesorectum in rectal cancer patients using gadofosveset-enhanced MRI, and investigate their relation to the node involvement and therapy response.

\section{Materials and Methods}

\section{Patients}

This prospective study was approved by the local review board and written informed consent was obtained from all the patients prior to their inclusion. Thirty primary rectal cancer patients treated with neoadjuvant chemoradiation (CRT) in our medical center between March 2011 and February 2012 were considered eligible. The following inclusion criteria were applied1) histopathologically proven rectal adenocarcinoma, 2) semicircular tumor on 
anatomical MRI, 3) glomerular filtration rate $>30 \mathrm{ml} / \mathrm{min} / \mathrm{kg}$ (GFR $<30$ is a contra-indication for gadofosveset), 4) neoadjuvant chemoradiation (50.4Gy in daily fractions of $1.8 \mathrm{~Gy}$ and $825 \mathrm{mg}$ / $\mathrm{m}^{2}$ oral capecitabine administered twice a day), 5) total mesorectal excision (TME) or in case of non-operative treatment and follow-up in patients with a clinical complete response - a disease-free follow-up period of $>1$ year. In total 17 patients were excluded for the following reasons; mucinous tumor ( $n=2$ ), circular tumor ( $n=5)$, ineligible for gadofosveset, i.e. GFR $<30$ $(n=4)$, no TME ( $n=5)$, or delayed TME $(n=1)$. The final study group consisted of 13 patients ( 1 woman and 12 men). The inclusion of patients with semicircular tumors was motivated by the fact that in these patients we could study the vascular abnormalities of mesorectal vasculature with respect to the mesorectum around normal rectal wall located within the same mesorectal segment (image slice). By this intraslice comparison, we avoided the intrinsic vascular differences between the distal, middle and proximal mesorectum. All the patients underwent primary staging MRI, followed by a long course of CRT. After a median interval of 10.1 weeks (range 8.6-14.9) from the end of CRT, 11 patients underwent TME and two patients were followed for $>1$ year according to a wait-and-see policy ${ }^{18,19}$.

To provide histological insights into the primary mesorectal status, we retrospectively identified another group of 8 patients with semicircular rectal tumors, who underwent TME after only a short-course radiotherapy (5 x 5Gy). The histological material was obtained from the Maastricht Pathology Tissue Collection. Collection, storage and use of tissue and patient data were performed in agreement with The Code for Proper Secondary Use of Human Tissue in the Netherlands. Both patient groups' characteristics is summarised in Table 1.

\section{MRI data acquisition}

MRI was performed on a 1.5T scanner (Philips Medical Systems, Netherlands) using a 5-element phased-array coil. The imaging protocol included T2-weighted, diffusionweighted and T1-weighted scans, which are routinely used in our medical center for staging and restaging of rectal cancer patients. DCE-MRI was performed at primary staging using a dynamic 3D T1-weighted gradient-echo scan (TRIVE) on a free-breathing patient, and using the following parameters: echo time $=4.6 \mathrm{~ms}$, repetition time $=7.9 \mathrm{~ms}$, time resolution $=8 \mathrm{~s}$ (time between consecutive DCE-MRI series), total scan time $=5 \mathrm{~min}$, pixel size $=0.43 \times 0.43 \times 5 \mathrm{~mm}^{3}$, field of view $=22 \times 22 \times 5.5 \mathrm{~cm}^{3}$. After the first three DCE-MRI series, gadofosveset trisodium (Ablavar ${ }^{\circledR}$, Lantheus Medical Imaging, USA), an FDA-approved blood pool contrast agent, was administered intravenously at the dose of $0.03 \mathrm{mmol} / \mathrm{kg}$ and rate of $0.7 \mathrm{ml} / \mathrm{s}$ using an injection system (Medrad ${ }^{\circledR}$ Spectris Solaris ${ }^{\circledR}$, MEDRAD, USA).

\section{MRI data analysis}

The clinical assessment of local tumor spread was based on standard T2-weighted images. Nodal staging was performed on gadofosveset-enhanced T1-weighted images according to previously described criteria ${ }^{20}$. A summary of patient clinical characteristics is presented in Table 1. 
Table 1. Patient group characteristics

\begin{tabular}{|c|c|c|c|}
\hline \multicolumn{2}{|l|}{ Parameters } & DCE-MRI $(n=13)$ & Histology $(n=8)$ \\
\hline \multicolumn{2}{|l|}{ Age [mean +/- SD] } & $66.2 \pm 9.3$ & $74 \pm 7.6$ \\
\hline \multirow{2}{*}{ Gender [n (\%)] } & Male & $12(92.3 \%)$ & $6(75 \%)$ \\
\hline & Female & $1(7.7 \%)$ & $2(25 \%)$ \\
\hline \multirow{2}{*}{ Clinical T-stage } & $\mathrm{cT} 2$ & $2(15.4 \%)$ & - \\
\hline & cT3 & $11(84.6 \%)$ & $8(100 \%)$ \\
\hline \multirow{3}{*}{ Clinical N-stage } & $\mathrm{CNO}$ & $4(31 \%)$ & $5(67.5 \%)$ \\
\hline & $\mathrm{CN} 1$ & $3(23 \%)$ & $1(12.5 \%)$ \\
\hline & $\mathrm{cN} 2$ & $6(46 \%)$ & $2(25 \%)$ \\
\hline \multirow{3}{*}{ Tumor orientation } & Anterior & $5(38.5 \%)$ & $4(50 \%)$ \\
\hline & Posterior & $2(15.4 \%)$ & $2(25 \%)$ \\
\hline & Lateral & $6(46.1 \%)$ & $2(25 \%)$ \\
\hline \multirow{2}{*}{ Neoadjuvant treatment } & $5 \times 5 \mathrm{~Gy}$ & 0 & $8(100 \%)$ \\
\hline & CRT & $13(100 \%)$ & 0 \\
\hline \multirow{6}{*}{ Pathologic T-stage } & (y)pT0 & 0 & 0 \\
\hline & (y)pT1 & $3(23 \%)$ & 0 \\
\hline & (y)pT2 & $4(30.8 \%)$ & $1(12.5 \%)$ \\
\hline & (y)pT3 & $4(30.8 \%)$ & $6(75 \%)$ \\
\hline & (y)pT4 & 0 & $1(12.5 \%)$ \\
\hline & (y)pTx & $2^{\wedge}(15.4 \%)$ & n.a. \\
\hline \multirow{4}{*}{ Pathologic N-stage } & (y)pN0 & $7(53.8 \%)$ & $6(75 \%)$ \\
\hline & (y)pN1 & $4(30.8 \%)$ & $2(25 \%)$ \\
\hline & (y)pN2 & 0 & 0 \\
\hline & (y)pNx & $2^{\wedge}(15.4 \%)$ & n.a. \\
\hline \multirow{6}{*}{ TRG } & 1 & 0 & n.a. \\
\hline & 2 & $5(38.5 \%)$ & n.a. \\
\hline & 3 & $4(30.8 \%)$ & n.a. \\
\hline & 4 & $1(7.7 \%)$ & n.a. \\
\hline & 5 & $1(7.7 \%)$ & n.a. \\
\hline & unknown & $2^{\wedge}(15.4 \%)$ & n.a. \\
\hline
\end{tabular}

CRT: long chemoradiotherapy with long interval, $5 \times 5 \mathrm{~Gy}$ : short radiotherapy;

TRG: tumor regression grade according to Mandard; ^ non-operated patients, n.a.: not applicable.

\section{Mesorectal microvasculature}

First, one reader (MHM) delineated the tumor on T2-weighted images using MRIcron software (by Chris Rorden, University of South Carolina, Columbia, South Carolina, USA).. The tumor ROls were subsequently overlaid on T1-weighted images. Only slices in which the tumor was visible were included for analysis. For these slices, the subtraction images were created by subtracting the baseline contrast (pre-contrast) from the post-contrast T1- weighted images obtained at 2 minutes from the start of enhancement, which reflect the steady-state contrast conditions ${ }^{21}$ (Matlab, Mathworks, USA) (Figure 1). 

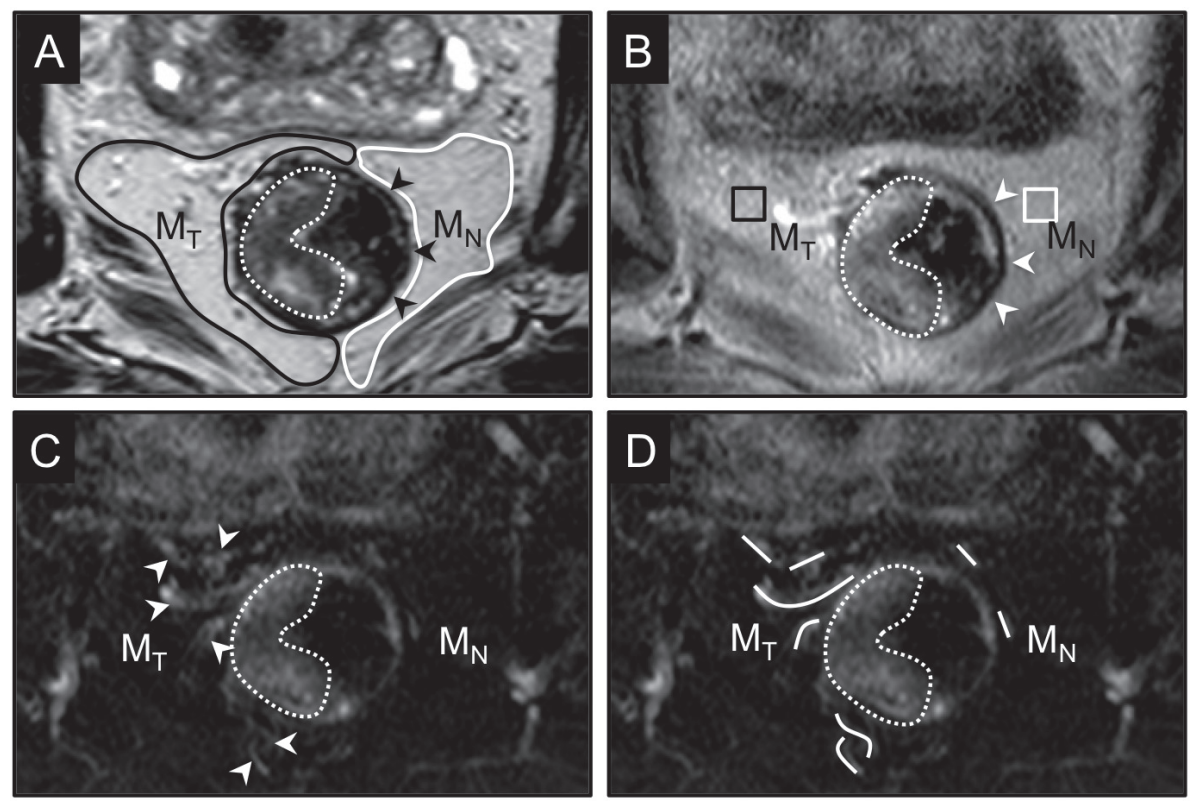

Figure 1. Definition of the tumor- and normal rectal wall-surrounding mesorectum.

(A) Representative T2-weighted image with the defined regions of interest in the tumor-surrounding $\left(M_{T}\right)$ (black line) and normal rectal wall-surrounding mesorectal fat $\left(\mathrm{M}_{\mathrm{N}}\right)$ (white line) used for the analysis of macrovasculature. Black arrowheads indicate the normal rectal wall and the white dashed line indicates the tumor. (B) Raw post-contrast T1-weighted image with the defined regions of interest in the tumor-surrounding (black squares) and normal mesorectal fat (white squares) used for the analysis of microvasculature. (C,D) Baseline-subtracted 2 min post-contrast T1-weighted image with the indicated vascular structures (white arrowheads) (C) and reader-defined vessel count/length (D).

Two independent readers (EK and MHM) assessed the macrovascular morphology by delineating the vessel branches, measurements of vessel diameter and length, and counting the number of vessels on subtraction images using Osirix software (www.osirix-viewer.com). During the analysis, T2-weighted MRI served as anatomical reference. The measurements were performed separately for the tumor-surrounding $\left(M_{T}\right)$ and normal mesorectum $\left(M_{N}\right) \cdot M_{T}$ was defined as a normal appearing adipose tissue, i.e, hyperintense on fat-unsuppressed T2w images, without tumor invasion or desmoplastic reaction, located ipsilateral of the tumor and extending from the tumor boundaries until the mesorectal fascia. $M_{N}$ was defined as the adipose tissue lying contralaterally to the tumor, neighboring the normal rectal wall and extending until the mesorectal fascia (Figure 1). Moreover, to reduce the impact of the mesorectal surface area that underwent analysis $\left(M_{T}\right.$ and $M_{N}$ was $50.2 \pm 27.8 \mathrm{~cm}^{2}$ and $33.6 \pm$ $16.2 \mathrm{~cm}^{2}$, respectively $\left.(p=0.07)\right)$, we also calculated the number of vessel branches $/ \mathrm{cm}^{2}$.

\section{Mesorectal microvasculature}

The microvascular function was assessed by using semiquantitative analysis of the dynamic T1w data. First, 3 regions of interest (ROI) were manually selected in the mesorectal fat in 
the surrounding of the tumor $\left(\mathrm{M}_{\mathrm{T}}\right)$ and normal rectal wall $\left(\mathrm{M}_{\mathrm{N}}\right)$ by one reader (EK) (Figure 1B) (MRIcron). Post-contrast T1w images were used to select exclusively microvascular tissue region and avoid larger mesorectal vessels. Semiquantitative analysis of the enhancementtime profile was performed using Matlab (Mathworks) on voxel-by-voxel basis. The start of enhancement was defined as a first timepoint of DCE-MRI series at which the signal intensity (SI) was increased $3 \times$ the standard deviation of the noise compared to the baseline SI. The baseline was defined as the average of the 7 pre-contrast timepoints. The SI increase was normalized to the baseline state, resulting in the relative signal enhancement. Subsequently, the area under the first 2 min enhancement-time curve (AUC) (arbitrary units (a.u.)) was calculated. The mean AUC calculated from all the included voxels of three ROIs was used as a semiquantitative indicator of the mesorectal microvascular function. We were not able to derive other parameters that we have previously used for characterisation of the tumor enhancement profile, such as the initial enhancement peak or initial and late enhancement slope ${ }^{22}$. This was due to the lack of (at least) three data points in the initial upslope enhancement phase and the lack of significant enhancement compared to noise level observed both early and late post-contrast, primarily for normal mesorectal data. Therefore, for consistency, we selected AUC as the most universal parameter. Samples of the obtained average enhancement-time curves are presented in Figure 2.
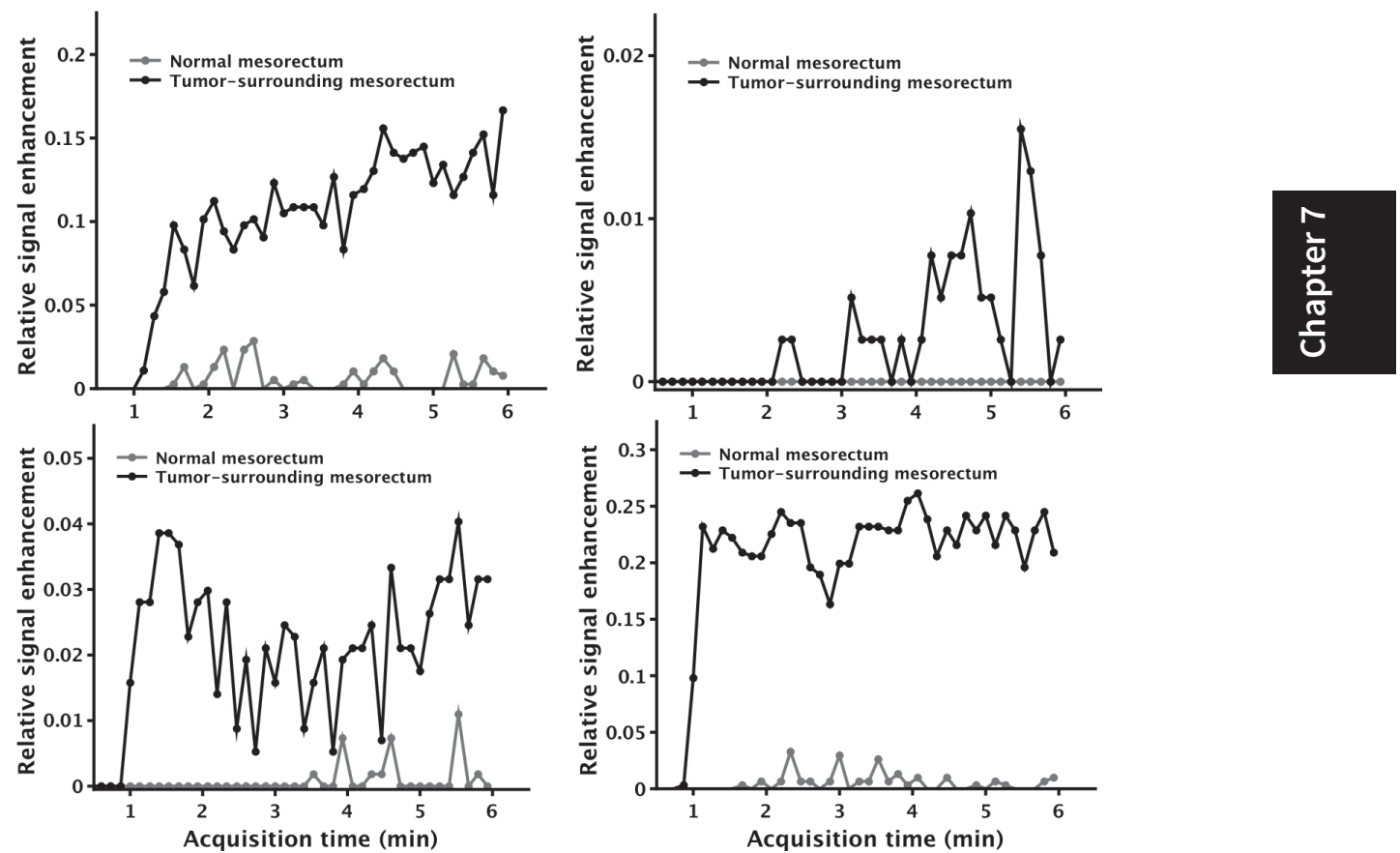

Figure 2.

Average relative signal enhancement-time curves obtained for four different patients for the tumor-surrounding (black lines) and normal mesorectum (grey lines). 


\section{Histopathological analysis}

The histopathological assessment of TME specimens of patients included in the prospective DCE-MRI study was performed by an oncological pathologist (RR) according to the method of Quirke et al. ${ }^{23}$. Tumor regression grade (TRG) was assessed according to the method described by Mandard et al. ${ }^{24}$. The patients with TRG $1-2$ and ypT0-1N0 were defined as good responders and patients with TRG 3-5 and ypT2-3N+ were defined as poor responders ${ }^{25}$. The non-operated patients included in the wait-and-see policy were considered as good responders.

The histological analysis of mesorectal vasculature was performed; $4 \mu \mathrm{m}$-thick sections of formalin-fixed and paraffin-embedded tumor and normal rectum specimens were cut. Before staining, the sections were deparafinized and rehydrated using xylene and decreasing concentrations of ethanol, followed by heat-induced antigen retrieval by using Tris/EDTA buffer pH=9 (K8004 EnVision ${ }^{\mathrm{TM}}$ Flex Target Retrieval Solution High pH 9) (Dako, Heverlee, Belgium) for $30 \mathrm{~min}$ at $97^{\circ} \mathrm{C}$. The sections were stained with either hematoxylin and eosin (H\&E) (Klinipath, Duiven, Netherlands) or CD31 antibody. In the latter case, the tissues were incubated with the monoclonal mouse anti-human CD31 antibody (Clone JC70A) (Dako). After 30 min of incubation with the primary antibody, the visualization of CD31 antigen was achieved with EnVision FLEX'M + Mouse (LINKER) kit (Dako). Hematoxylin (Dako) was used as a nuclear counterstaining.

The mesorectal macrovasculature was assessed from H\&E-stained mesorectal specimens, which were scanned using VENTANA iScan HT Scanner (Ventana Medical Systems, Inc., Tucson, AZ, USA) and analyzed with Image Viewer v. 3.1.2. software (Ventana Medical Systems). The blood vessels were defined by the presence of smooth muscle layer (tunica media) surrounded by the connective tissue (tunica adventitia) and frequently residual red blood cells in the vessel lumen. For consistency with the MR image-based analysis, only vessels with a diameter of approximately $1 \mathrm{~mm}$ and higher were included in the analysis. Due to a low number of macrovascular structures found in the mesorectal fat specimens, i.e, 2-4 vessels per tissue section, only the vascular diameter, measured in the widest vessel dimension, was used for statistical analysis.

The analysis of mesorectal microvasculature was performed on CD31-stained sections by using bright field microscopy (Leica DM3000, Leica Microsystems, Wetzlar, Germany) at 200x magnification. On average, 10 microscopy images were acquired (Camera Leica DFC 320, Leica Microsystems) per tissue section. The fraction of CD31-positive tissue area was determined using a custom-made Matlab script (Mathworks). In short, the color threshold was manually adjusted to extract the brown-stained tissue area. Subsequently, based on the created image mask, the percentage of CD31-positive tissue area was calculated. The average percentage of CD31-positive tissue area was calculated from all the analyzed images. 


\section{Statistical analysis}

All results are reported as mean \pm standard deviation (SD). Normality of the distribution of all the studied parameters was tested using the Shapiro-Wilk test ${ }^{26}$. The comparison of tumor-surrounding and normal mesorectum with respect to image- and histology-based macro- and microvascular parameters was performed by using a paired $t$-test. The same test was used to determine chemoradiotherapy-induced changes in the image-based vascular parameters. The correlation between the image-based macrovascular parameters assessed by two independent readers was tested by using intraclass correlation (model: two-way mixed, type: absolute agreement). Furthermore, a t-test was used to compare the vascular parameters of patients with respect to nodal involvement ( $\mathrm{CN}$ ) and pathological response to CRT (TRG and (y)pTN). All statistical analyses were performed using SPSS 16.0 (SPSS Inc., Chicago, IL, USA). The difference was considered significant at $p<0.05$.

\section{Results}

\section{Mesorectal macrovascular morphology}

There were significantly more vessel branches per image slice in the tumor-surrounding mesorectum $\left(M_{T}\right)$ compared to the counterpart around normal rectal wall $\left(M_{N}\right)$ reported by both readers, i.e., reader 1: $9.0 \pm 4$.3 versus $1.7 \pm 1.2$ vessels, $p_{1}=0.001$; and reader $2: 7.3 \pm 3.4$ versus
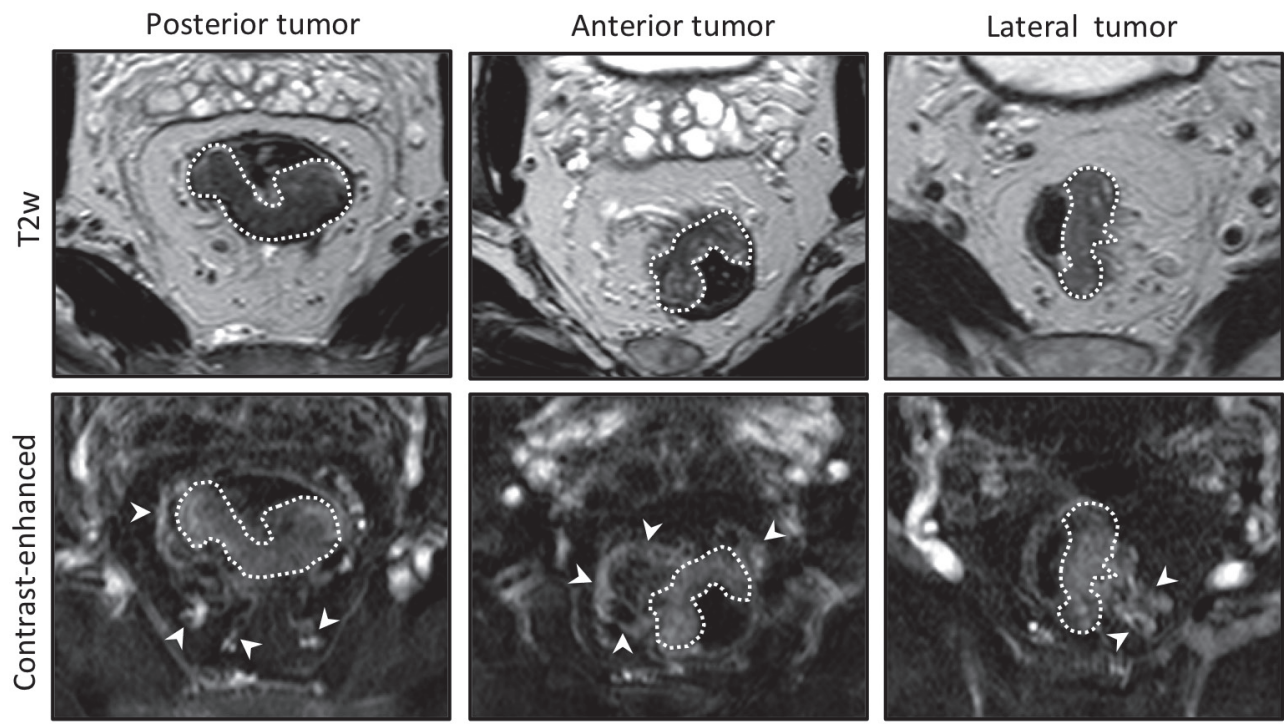

\section{Figure 3.}

T2-weighted (upper panel) and subtraction images (lower panel) from patients with different orientation of rectal tumor. The macroscopic vascular structures are indicated with white arrowheads. 
1.7 \pm 1.4 vessels, $p_{2}=0.002$ (Table 2). This vascular asymmetry was found in all 13 patients, independent of the tumor orientation in the rectum (Figure 3). Consistently, we found a significantly higher number of vessels per $\mathrm{cm}^{2}$ of $\mathrm{M}_{\mathrm{T}}$ compared to the normal counterpart $\mathrm{M}_{N}$ (Table 2). Furthermore, both readers found approximately $0.2 \mathrm{~mm}$ larger vessel diameter in $M_{T}$ $\left(p_{1}=0.025, p_{2}=0.038\right)$ (Table 2$)$. The vessel length was similar in both mesorectal compartments (Table 2). We found a strong interobserver correlation for the number of vessel branches, i.e., the intraclass correlation coefficient (ICC) was 0.94, p<0.0001, and moderate correlation for the vessel length (ICC $=0.46, p=0.021$ ). However, there was no significant correlation between the vascular diameter measurements (ICC=0.34, $\mathrm{p}=0.092$ ).

Table 2. Comparison between the tumor- and normal rectal wall-surrounding mesorectum with respect to macro- and microvascular vascular parameters.

\begin{tabular}{|c|c|c|c|c|c|c|}
\hline & \multicolumn{2}{|c|}{$\mathrm{M}_{\mathrm{T}}$} & \multicolumn{2}{|c|}{$M_{N}$} & \multicolumn{2}{|c|}{ p-value } \\
\hline & $\mathbf{R 1}$ & $\mathbf{R 2}$ & $\mathbf{R 1}$ & $\mathbf{R 2}$ & R1 & $\mathbf{R 2}$ \\
\hline \multicolumn{7}{|c|}{ Macrovascular parameters } \\
\hline $\begin{array}{l}\text { Average number of } \\
\text { vessels }\end{array}$ & $9.0 \pm 4.3$ & $7.3 \pm 3.4$ & $1.7 \pm 1.2$ & $1.7 \pm 1.4$ & $0.001^{*}$ & $0.002^{*}$ \\
\hline $\begin{array}{l}\text { Number of } \\
\text { vessels } / \mathrm{cm}^{2}\end{array}$ & $0.9 \pm 0.4$ & $0.8 \pm 0.4$ & $0.3 \pm 0.3$ & $0.3 \pm 0.2$ & $0.0003^{\star}$ & $0.002^{*}$ \\
\hline $\begin{array}{l}\text { Average vessel } \\
\text { diameter (mm) }\end{array}$ & $1.6 \pm 0.2$ & $1.7 \pm 0.2$ & $1.3 \pm 0.4$ & $1.6 \pm 0.3$ & $0.025^{\star}$ & $0.038^{\star}$ \\
\hline $\begin{array}{l}\text { Average vessel length } \\
(\mathrm{mm})\end{array}$ & $7.1 \pm 2.2$ & $8.5 \pm 2.4$ & $5.3 \pm 0.8$ & $7.4 \pm 1.3$ & 0.15 & 0.245 \\
\hline \multicolumn{7}{|c|}{ Microvascular parameter } \\
\hline AUC (a.u.) & \multicolumn{2}{|c|}{$6.4 \pm 6.6$} & \multicolumn{2}{|c|}{$0.6 \pm 1.0$} & \multicolumn{2}{|c|}{$0.01^{*}$} \\
\hline
\end{tabular}

$\mathrm{M}_{\mathrm{T}}$ : tumor-surrounding mesorectum, $\mathrm{M}_{\mathrm{N}}$ : normal rectal wall-surrounding mesorectum, R1: reader 1, R2: Reader 2, a.u: arbitrary units, * indicates significant difference

\section{Mesorectal microvascular function}

In most cases, $\mathrm{M}_{\mathrm{T}}$ displayed a considerable contrast enhancement, which persisted over time (Figure 2). In contrast, in $\mathrm{M}_{\mathrm{N}}$, the enhancement was very low and stable over time or absent. Consistently, $A \cup C$ of $M_{T}$ were significantly higher compared to $A \cup C$ of $M_{N}$, i.e, 6.4 \pm 6.6 a.u. and 0.6 \pm 1.0 a.u., respectively ( $p=0.01$ ) (Table 2), although a large interpatient variability was observed.

\section{Mesorectal vasculature and lymph nodes}

The tumor-surrounding mesorectum of patients with malignant nodes (cN1-2) displayed on average two-fold higher vascular branching $\left(p_{1}=0.005, p_{2}=0.03\right)$ and $0.2 \mathrm{~mm}$ larger vascular diameter $\left(p_{1}=0.008, p_{2}=0.02\right)$ compared to that of patients with benign mesorectal nodes (cN0) (Table 3). However, the macrovascular length and microvascular function were similar 
in both patient groups. Furthermore, there were no differences with respect to the vascular parameters of $\mathrm{M}_{\mathrm{N}}$.

\section{Mesorectal vasculature in good and poor responders to CRT}

At the primary staging, thus before therapy, good responders (TRG1-2) had significantly higher number of vessel branches in $M_{T}$ compared to poor responders (TRG3-5): reader 1: 11.9 \pm 3.5 versus $5.6 \pm 1.5$ vessels and reader $2: 9.5 \pm 3.5$ versus $5.6 \pm 1.5$ vessels $\left(p_{1} / p_{2}=0.02\right)$. A similar trend was found when using $(y)$ pTN restaging criteria $\left(p_{1}=0.07, p_{2}=0.09\right)$. Other macrovascular parameters and microvascular AUC showed no significant association with the response to CRT, or, in the case of vascular diameter, only one reader reached the significance level (Table 3).

A Tumor-surrounding mesorectum
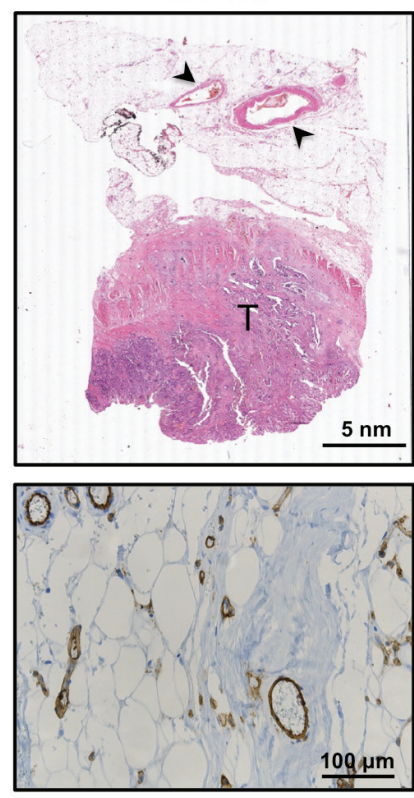

Normal mesorectum
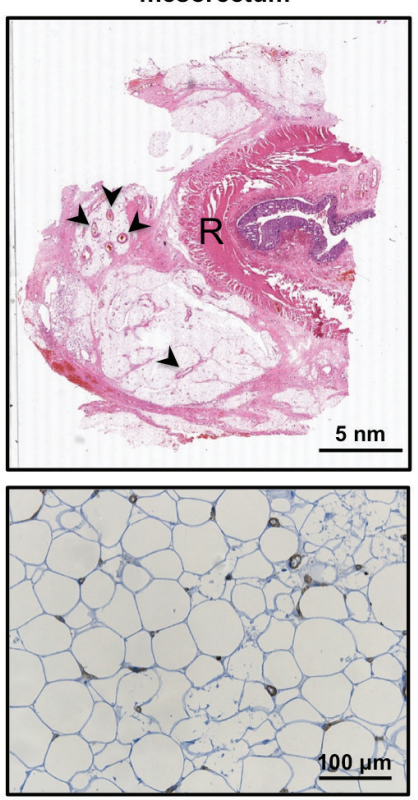

B

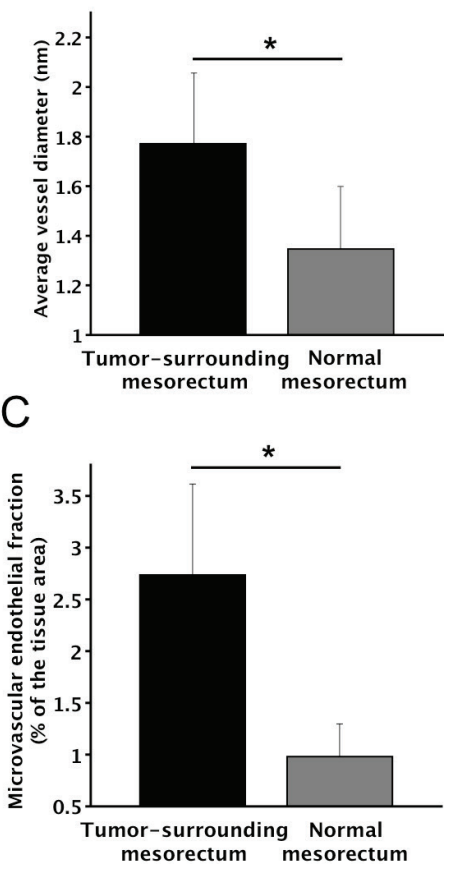

Figure 4. Histological evaluation of mesorectal vasculature at the primary tumor stage.

(A) In upper panel, the representative images of hematoxylin and eosin-stained sections obtained from the total mesorectal excision specimens display the tumor ( $T$ ) (upper left image) and normal rectal wall (R) (upper right image) with the surrounding mesorectal fat. Black arrows depict some of large mesorectal vascular structures. In lower panel, the representative microscopy images of the tumor-surrounding (lower left image) and normal mesorectum (lower right image) with the CD31-stained microvacular endothelium (brown) and hematoxylin costaining (blue). (B,C) The comparison between the tumor-surrounding and normal mesorectum with respect to the average macrovascular diameter (B) and microvascular endothelial fraction (C). Bars represent mean \pm SD. Symbol "*” indicates a significant difference $(P<0.05)$. 


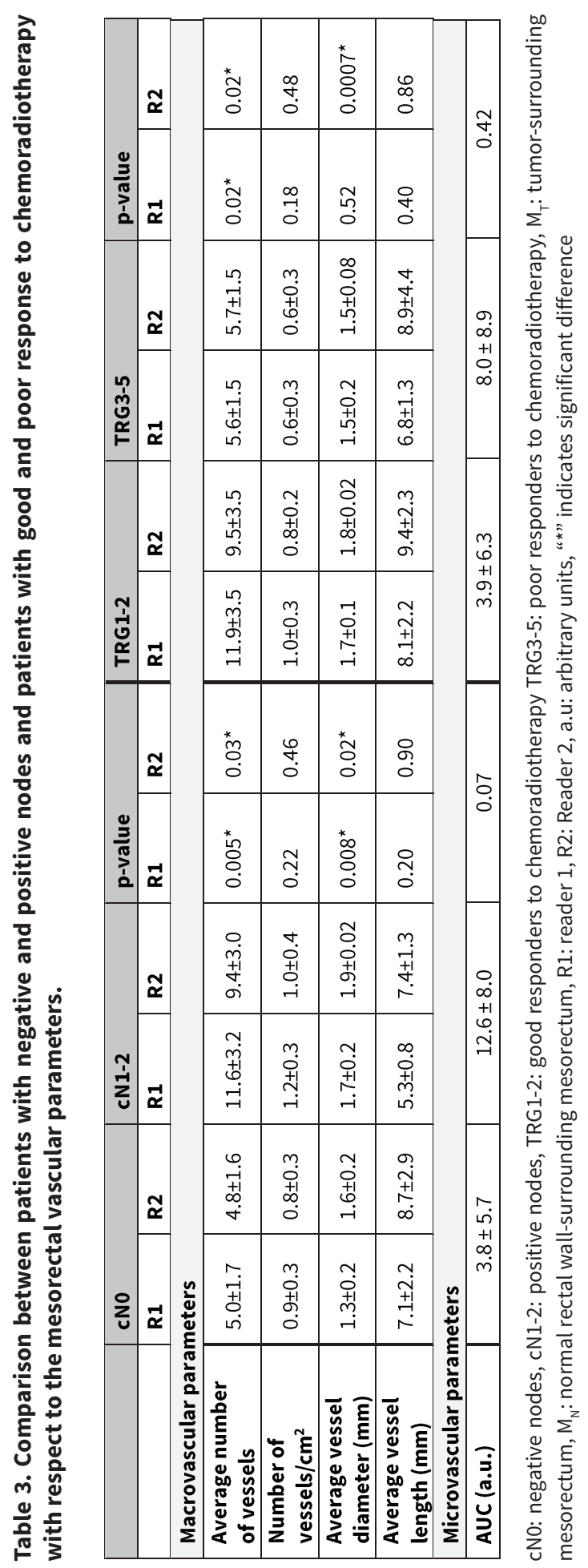




\section{Histological evaluation of mesorectal vasculature}

Histopathology of the 8 patients treated with short course 5x5Gy followed by TME revealed a significantly higher vascular diameter in the tumor-surrounding mesorectum $M_{T}(1.8 \pm 0.3 \mathrm{~mm})$ compared to the normal counterpart $M_{N}(1.3 \pm 0.25 \mathrm{~mm})$. Figure $4 \mathrm{~A}$ (upper panel) displays representative images of a tumor and normal rectal wall with the surrounding mesorectal fat, containing large mesorectal vessels ( $p=0.027$ ). The microscopical assessment of CD31-stained sections (Figure 4A, lower panel) revealed a significantly higher microvascular endothelial fraction of $2.7 \pm 0.9 \%$ in tumor-surrounding mesorectum $M_{T}$ compared to $0.98 \pm 0.3 \%$ in the normal mesorectum $M_{N}(p=0.0005)$ (Figure $\left.4 C\right)$.

\section{Discussion}

The aim of this study was to investigate macro- and microvascular parameters of the mesorectum and their relation to the node involvement and therapy response. The proposed vascular MRI method revealed a highly complex macrovascular structure and highly elevated microvascular function in the tumor-surrounding mesorectum, i.e., a two- to ten-fold higher vessel branching, circa $0.2 \mathrm{~mm}$ higher vascular diameter and ten-fold higher AUC compared to the normal wall surrounding mesorectum. Histological evaluation of the resection specimens showed a similar trend in the mesorectal vascular profile, i.e., an approximately $0.5 \mathrm{~mm}$ higher macrovascular diameter and 60\% higher microvascular endothelial fraction in the tumor-surrounding mesorectum. Interestingly, high primary macrovascular parameters were significantly associated with nodal involvement and favorable therapy response.

The tumor-surrounding mesorectal vasculature displayed several abnormal features, including a high vascular branching, enlarged vascular radii and enhanced microvascular function compared to the mesorectum around normal rectal wall. This vascular profile was supported by both imaging (Table 2) and histological parameters (Figure 4). Notably, the tumor-associated vascular enhancement was observed independently of the tumor orientation in the rectum (Figure 3). Considering the posterior location of superior rectal artery ${ }^{27}$, one might expect the preference of vascular enhancement in posterior tumors. However, our data strongly suggests an angiogenic origin (tumor-induced) of the peritumoral vasculature and a limited impact of rectal vascular anatomy. A similarly enhanced vascular profile of peri-tumoral tissue areas was previously described in brain and breast cancer patients ${ }^{10,11,28}$. Crucially, however, there are no previous reports on the impact of rectal tumor on host tissue macro- and microvascular properties. Thanks to the application of gadofosveset, an FDA-approved albumin-binding blood pool contrast agent, we were able to generate both the dynamic (functional) and equilibrium phase (steady-state) (structural) data during one DCE-MRI scan, which is more time- and cost-effective compared to the previously proposed multi-scan approach ${ }^{29}$.

Among the investigated macrovascular parameters, the vascular branching around the tumor was found to be the most promising, both considering its significant association with the nodal state and therapy response, and excellent interobserver agreement. Although 
both readers observed differences in the vascular diameter, these were below the spatial resolution of DCE-MRI $(0.43 \times 0.43 \mathrm{~mm}$, in-plane) and led to large interobserver discrepancies. The detection of these sub-resolution differences was possible due to the application of pseudo-continuous scale ROI tools of Osirix image software. Therefore, they cannot be considered as fully reliable. Nevertheless, our findings on the enhanced vascular branching coincide with previous non-contrast magnetic resonance angiography (MRA) studies, which showed abnormalities of major cerebral vessels lying outside tumor boundaries ${ }^{28}$, and their potential prognostic and predictive implications ${ }^{12,30}$. In breast cancer, gadobenate dimeglumine-enhanced MRA revealed one-sided increased vascularity associated with ipsilateral invasive carcinoma ${ }^{11}$. Both our and the aforementioned studies demonstrated that the cancer-associated angiogenesis is not limited to microvasculature but also involves large vessels, and that blood pool imaging represents a potentially useful strategy in cancer diagnostics. Furthermore, our findings suggest that the remodeling of mesorectal vasculature occurs in a paracrine manner, presumably mediated by pro-angiogenic factors and chemokines released by tumor cells into the surrounding tissue ${ }^{7,31}$. Consequently, the mesorectal vascular status might be closely linked to the tumor angiogenic activity and its metabolic demands.

Our findings also carry potential clinical implications for patients with rectal cancer. Positive extramural venous invasion (EMVI) and nodal malignancy are known poor prognostic factors for rectal cancer. Yet assessment of EMVI and nodal stage by imaging is difficult and micrometastases remain beyond the image resolution. Considering our initial findings on the relation between high mesorectal vascular parameters and nodal involvement (Table 3), and the co-localization of tumor microlesions with mesorectal vessels, the mesorectal macrovasculature parameters should be further investigated as potential prognostic markers in rectal cancer. Previously, Sica et al. ${ }^{10}$ reported that brain cancer patients with hypervascular peritumor tissue have a significantly shorter survival time and an about 3.5-fold increase in death risk. Moreover, we observed that highly developed mesorectal vasculature predispose to better response to CRT (Table 3). Possibly, the tumor-surrounding mesorectal vessels also contribute to the tumor oxygenation and facilitate delivery of cytotoxic agents, which increases radiosensitivity. Therefore, the assessment of mesorectal vasculature may potentially help to select patients that would benefit from the radiation dose escalation ${ }^{32}$. Notably, we found no association between microvascular AUC and therapy response, which is in line with our previous tumor findings ${ }^{22}$. The late slope of enhancement, which we have previously shown as a potential predictive tumor marker ${ }^{22}$, was unfortunately not easy to quantify for the mesorectal fat tissue (Figure 2).

A limitation of our study is a low number of patients and the lack of clinical endpoints to determine the diagnostic performance of mesorectal vascular parameters. Furthermore, we have no vascular data from control subjects, who would potentially provide better representation of the normal mesorectal vasculature. The reader-based analysis of mesorectal vascular maps was subjective and time consuming. The clinical application of mesorectal 
vascular mapping would require the development of (semi)automated and objectivized method of vascular assessment. Additionally, the differences in macrovascular diameter were smaller than the spatial resolution of DCE-MRI, which resulted in a poor interobserver agreement. The method application might be limited by the extent of tumor spread into the mesorectum and EMVI, which would mask the presence of mesorectal vasculature. Finally, we had no proper histological validation of the vascular imaging data at the primary cancer staging, since the mesorectal tissue specimens were available only post-CRT.

In conclusion, our study demonstrated that the formation of highly vascular stroma in the mesorectum of rectal cancer patients precedes the actual tumor invasion. The mesorectal vascular enhancement was observed both at the macro- and microscopic level. Moreover, MRI-based mesorectal macrovascular parameters showed significant association with the primary node involvement and tumor regression after CRT. Further research with a larger patients group is warranted to confirm the diagnostic value of the proposed mesorectal vascular parameters.

\section{References}

1. Folkman J. Tumor angiogenesis: therapeutic implications. N Engl J Med 1971; 285(21): 1182-6.

2. Ferrara N, Kerbel RS. Angiogenesis as a therapeutic target. Nature 2005; 438(7070): 967-74.

3. Weidner N, Semple JP, Welch WR, Folkman J. Tumor angiogenesis and metastasis--correlation in invasive breast carcinoma. The New England journal of medicine 1991; 324(1): 1-8.

4. Weidner N, Carroll PR, Flax J, Blumenfeld W, Folkman J. Tumor angiogenesis correlates with metastasis in invasive prostate carcinoma. The American journal of pathology 1993; 143(2): 401-9.

5. Li CY, Shan S, Huang Q, et al. Initial stages of tumor cell-induced angiogenesis: evaluation via skin window chambers in rodent models. Journal of the National Cancer Institute 2000; 92(2): 143-7.

6. Nowacki P, Kojder I. Peritumoral angiogenesis around primary and metastatic brain neoplasms. Morphometric analysis. Folia neuropathologica / Association of Polish Neuropathologists and Medical Research Centre, Polish Academy of Sciences 2001; 39(2): 95-102.

7. Yu DC, Chen J, Ding YT. Hypoxic and highly angiogenic non-tumor tissues surrounding hepatocellular carcinoma: the 'niche' of endothelial progenitor cells. International journal of molecular sciences 2010; 11(8): 2901-9.

8. Isaka N, Padera TP, Hagendoorn J, Fukumura D, Jain RK. Peritumor lymphatics induced by vascular endothelial growth factor-C exhibit abnormal function. Cancer research 2004; 64(13): 4400-4.

9. Winkler F, Kienast Y, Fuhrmann M, et al. Imaging glioma cell invasion in vivo reveals mechanisms of dissemination and peritumoral angiogenesis. Glia 2009; 57(12): 1306-15.

10. Sica G, Lama G, Anile C, et al. Assessment of angiogenesis by CD105 and nestin expression in peritumor tissue of glioblastoma. International journal of oncology 2011; 38(1): 41-9. 
11. Sardanelli F, lozzelli A, Fausto A, Carriero A, Kirchin MA. Gadobenate dimeglumine-enhanced MR imaging breast vascular maps: association between invasive cancer and ipsilateral increased vascularity. Radiology 2005; 235(3): 791-7.

12. Bullitt E, Zeng D, Gerig G, et al. Vessel tortuosity and brain tumor malignancy: a blinded study. Academic radiology 2005; 12(10): 1232-40.

13. Smith NJ, Barbachano Y, Norman AR, Swift RI, Abulafi AM, Brown G. Prognostic significance of magnetic resonance imaging-detected extramural vascular invasion in rectal cancer. The British journal of surgery 2008; 95(2): 229-36.

14. Smith NJ, Shihab O, Arnaout A, Swift RI, Brown G. MRI for detection of extramural vascular invasion in rectal cancer. AJR American journal of roentgenology 2008; 191(5): 1517-22.

15. Betge J, Pollheimer MJ, Lindtner RA, et al. Intramural and extramural vascular invasion in colorectal cancer: prognostic significance and quality of pathology reporting. Cancer 2012; 118(3): 628-38.

16. Leiner T, Goyen M, Rohrer M, Schönberg SO. Clinical blood pool MR imaging: Springer; 2008.

17. Turetschek K, Floyd E, Helbich T, et al. MRI assessment of microvascular characteristics in experimental breast tumors using a new blood pool contrast agent (MS-325) with correlations to histopathology. Journal of Magnetic Resonance Imaging 2001; 14(3): 237-42.

18. Maas M, Nelemans PJ, Valentini V, et al. Long-term outcome in patients with a pathological complete response after chemoradiation for rectal cancer: a pooled analysis of individual patient data. The lancet oncology 2010; 11(9): 835-44.

19. Maas M, Beets-Tan RG, Lambregts DM, et al. Wait-and-see policy for clinical complete responders after chemoradiation for rectal cancer. J Clin Oncol 2011; 29(35): 4633-40.

20. Lambregts DM, Beets GL, Maas M, et al. Accuracy of gadofosveset-enhanced MRI for nodal staging and restaging in rectal cancer. Annals of surgery 2011; 253(3): 539-45.

21. Leiner T, Goyen M, Rohrer M, Schönberg S. Clinical blood pool MR imaging: Springer; 2008.

22. Martens MH, Subhani S, Heijnen LA, et al. Can perfusion MRI predict response to preoperative treatment in rectal cancer? Radiother Oncol 2015; 114(2): 218-23.

23. Quirke P, Durdey P, Dixon MF, Williams NS. Local recurrence of rectal adenocarcinoma due to inadequate surgical resection. Histopathological study of lateral tumour spread and surgical excision. Lancet 1986; 2(8514): 996-9.

24. Mandard AM, Dalibard F, Mandard JC, et al. Pathologic assessment of tumor regression after preoperative chemoradiotherapy of esophageal carcinoma. Clinicopathologic correlations. Cancer 1994; 73(11): 2680-6.

25. Vecchio FM, Valentini V, Minsky BD, et al. The relationship of pathologic tumor regression grade (TRG) and outcomes after preoperative therapy in rectal cancer. International journal of radiation oncology, biology, physics 2005; 62(3): 752-60.

26. Shapiro SS, Wilk MB. An analysis of variance test for normality (complete samples). Biometrika 1965; 52(3/4): 591-611.

27. Moore KL, Dalley AF, Agur AM. Clinically oriented anatomy: Lippincott Williams \& Wilkins; 2013.

28. BullittE, Lin NU, Smith JK, et al. Blood vessel morphologic changes depicted with MR angiography during treatment of brain metastases: a feasibility study. Radiology 2007; 245(3): 824-30. 
29. Parikh AH, Smith JK, Ewend MG, Bullitt E. Correlation of MR perfusion imaging and vessel tortuosity parameters in assessment of intracranial neoplasms. Technology in cancer research \& treatment 2004; 3(6): 585-90.

30. Bullitt E, Ewend MG, Aylward S, et al. Abnormal vessel tortuosity as a marker of treatment response of malignant gliomas: preliminary report. Technology in cancer research \& treatment 2004; 3(6): 577-84.

31. Zhang L, Yang N, Park JW, et al. Tumor-derived vascular endothelial growth factor up-regulates angiopoietin-2 in host endothelium and destabilizes host vasculature, supporting angiogenesis in ovarian cancer. Cancer research 2003; 63(12): 3403-12.

32. Galvin JM, De Neve W. Intensity modulating and other radiation therapy devices for dose painting. Journal of clinical oncology : official journal of the American Society of Clinical Oncology 2007; 25(8): 924-30. 


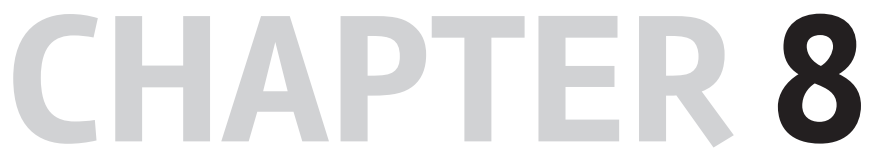

General discussion 
The standard treatment for locally advanced rectal cancer is neoadjuvant chemoradiotherapy and total mesorectal excision (TME). However there is a trend towards organ-preserving options for patients that respond very well to chemoradiotherapy. This approach is still considered controversial and is not yet standard practice. It is investigated in several trials with close surveillance and registry of patients. Accurate selection of patients is critical to minimize over- and undertreatment. Those in favor of organ-preservation, consider absence of residual tumor in the resection specimen after TME as overtreatment. On the other hand, incomplete response at initial assessment carries the risk for undertreatment and early local regrowth (within the first year of follow-up) if organ-preservation was opted for. Possibly longterm prognosis could be influenced by this over- and undertreatment. The aim of this thesis was to evaluate the oncological and functional outcome of organ-preserving treatments. A second aim was to investigate modern MRI techniques for improved response assessment after therapy, and response prediction before the onset of therapy to allow for optimized selection of patients eligible for organ-preservation.

\section{Organ preservation}

The first reports on organ-preservation were from the group of Habr-Gama ${ }^{1,2}$. Patients without evidence of residual disease at digital rectal examination (DRE) combined with endoscopy (and biopsies) were selected for a non-operative 'watch-and-wait' strategy (W\&W). Their most recent paper reported a high clinical complete response (CCR) rate of $49 \%$, but the local regrowth rate also increased to $31 \%{ }^{3}$. Similar results were reported in smaller series ${ }^{4-6}$. Other groups reported series of organ-preservation for good responders to neoadjuvant therapy with a local excision or transanal endoscopic microsurgery (TEM) of the scar tissue ${ }^{7-9}$. The main benefit of TEM is the histopathological confirmation of the response, a downside is the relatively high complication rate ${ }^{10,11}$. In the pilot study on W\&W from our center by Maas et al. ${ }^{5}$, patient selection was performed with DRE and endoscopy according to the methods described by Habr-Gama, but MRI including diffusion-weighted MRI (DWI) and gadofosvesetMRI was also incorporated. Twenty-one patients with a cCR were included in the watch-andwait policy (W\&W) and underwent follow-up including DRE, endoscopy, and MRI every 3 months during the first year, and every 6 months during the next 4 years. With strict selection criteria, i.e. (1) substantial downsizing with no residual tumor or residual fibrosis only on MRI (with low signal on DWI), (2) no suspicious lymph nodes on MRI (incl. gadofosveset-MRI), (3) no residual tumor at endoscopy or a white scar, (4) negative biopsies from the scar, and (5) no palpable tumor, only one out of $21 \mathrm{~W} \& W$ patients developed a local regrowth and 2-year overall survival was $100 \%$. While these criteria were highly specific for a complete response, they were not very sensitive, and a substantial number of patients with a complete response were not recognized and still underwent major TME surgery. In chapter 2 the same selection criteria for organ-preservation were applied in a larger patient cohort ( $n=100)$, but now patients with a 'near complete response', i.e. not meeting 1 or 2 criteria for a cCR (for example: small residual erythematous ulcer on endoscopy, residual tumor on standard MRI, 
but cCR based on DWI and endoscopy, or equivocal lymph nodes), could still be offered organ-preservation. These patients were given the following options: TME, TEM, or a second response assessment after 3 months. Patients that had a CCR at this second assessment, were offered the watch-and-wait policy at that time. All patients that still did not meet all criteria for cCR, e.g. persistent ulcer on endoscopy, or persistent residual tumor on MRI, were referred for TME. Of the in total 85 watch-and-wait patients (including the 21 patients from the pilot study), 24 were included at the second assessment after 3 months. Although not statistically different, 3-year local regrowth free survival was lower in patients included after the second assessment compared to those directly included at first assessment (77\% vs 88\%), suggesting that a larger proportion of the 'near complete responders' have residual tumor as compared to the 'complete responders'. The main concern is that these patients could have a decreased oncological outcome, e.g. higher rates of distant metastasis. In our current cohort however, we did not observe a difference in metastasis free survival and overall survival. This knowledge allows to further establish a right balance between an acceptable rate of false positives (missed residual tumor, i.e. local regrowths) and an acceptable rate of false negatives (overlooked complete response, i.e. pCR after resection). Further improvement of the response assessment after neoadjuvant therapy could further reduce both false negative and positive rates.

The main benefit of organ-preservation for the patient is avoiding the perioperative morbidity and mortality of major surgery, avoiding the need for a (permanent) colostomy, and avoiding the long-term anorectal dysfunction that can follow major surgery. This latter aspect has not been well studied, and so far only Maas et al. reported less fecal incontinence and better bowel function in W\&W patients compared to patients with a pCR ${ }^{5}$. In chapter 2 we observed that only one of the $20 \mathrm{~W} \& W$ patients suffered from major incontinence compared to 3 out of 7 in the TEM group. Even more, we conducted a retrospective study comparing the long-term quality of life (QoL) and the functional outcome of W\&W patients to case-matched patients that underwent TME and found that W\&W patients scored better on several domains ${ }^{12}$. Subsequently, the QoL and functional outcome is now monitored prospectively at shortterm and long-term follow-up.

\section{Response assessment with imaging}

The main problem with the use of subjective evaluation of morphologic MR features of residual disease is the interpretation of post-radiation fibrosis. Fibrotic tissue with and without residual small tumor volumes look similar on standard T2-weighted MRI. Areas of apparent fibrosis may still contain tumor cells in $50 \%$ of the cases ${ }^{13}$. Therefore, a visual assessment on T2W MRI is not accurate to differentiate between residual tumor and complete response, with an overall accuracy of only $43 \% 14$.

One of our research questions was whether objective measurements of the size and volume of the post-radiation remnant of the tumor bed (i.e. fibrosis and residual tumor) in addition to the simple visual interpretation of the images could improve the assessment of response. 
In chapter 3 we first reviewed all literature on tumor size and volume measurements based on standard T2W images. Then we applied the cut-offs derived from this literature in an independent multicenter cohort to validate them. Measuring only tumor length or size in three dimensions was not accurate to assess tumor response, but measuring the volume by manual delineation of the entire tumor resulted in improved results. The best overall accuracy was obtained for volume measurements on the post-CRT MRI and the rate of volume reduction after CRT. Both cut-offs reached a high specificity to detect a complete response, but sensitivities remained low (14-28\%), indicating that many patients with a pCR will be incorrectly diagnosed as having residual tumor and - using only volume measurements as a criterion will thus be denied the option for organ-preservation. Additionally volume measurements on post-CRT MRI are prone to delineation errors. For example, fibrosis that could potentially harbor residual tumor could be excluded from the delineated tumor volume or vice versa. The question is whether there are imaging biomarkers that can combine morphology with underlying tumor biology and thereby improve the response assessment. One of the most frequently applied functional MRI technique for response assessment is diffusion-weighted imaging (DWI) MRI, which focuses on differences in cellular density between tissues. A visual evaluation of residual high signal on DWI after treatment indicates residual tumor, and is easy applicable in clinics. The use of DWI for response evaluation has even been recommended in an European consensus paper ${ }^{15}$. However, the sensitivity for detecting a complete response remains low $(19 \%)^{16}$, although it increases to $65-83 \%$ when combined with a quantification of the volume of the residual high signal ${ }^{17,18}$. Nevertheless, even with DWI difficulties in the assessment of post-radiation fibrosis still results in an overestimation of the tumor remnant and an additional tool to quantify this fibrosis could be useful.

Magnetization transfer (MT) imaging is such a functional imaging technique that could quantitatively assess fibrosis. Fibrosis (e.g. collagen) contains many macromolecules, and as MT imaging is based on the presence of macromolecules it therefore has the potential to differentiate between fibrosis and residual tumor. In a proof of principle study (chapter 4) we showed that the MT ratio was significantly higher in histologically confirmed areas of fibrosis compared to areas of residual tumor, normal rectal wall, or edematous rectal wall, confirming our hypothesis. The potential of MT imaging was further explored on a patient level in chapter 5. Results showed that MT imaging can differentiate between patients with a good response (TRG1-2) and poor response (TRG3-5) with AUCs up to 0.88. Yet interobserver agreement was moderate, which could be partially explained by the study methodology. Regions of interest were placed directly on the MTR map with the T2-weigthed MRI as an anatomical reference. The MTR maps lack anatomical detail, which makes it challenging to interpret them. Also, the measurements were rather time consuming, but this could potentially be solved by the development of (semi)automated delineation tools. 


\section{Response prediction rationale}

Our patients series, as well as the series of Sao Paulo, included mostly patients with larger and distal tumors, for which CRT is a well-accepted standard neoadjuvant treatment. The clinical complete response rates in these larger tumors are generally around 10-20\%. Parallel to this, a number of studies have explored CRT in smaller tumors, with the explicit goal to achieve organ preservation. In this group of patients with generally T1-T2 (or early T3) and node negative tumors, neoadjuvant CRT followed by a local excision or TEM resulted in PCR rates of $32-38 \%$ and organ-preservation was possible for $49-66 \%$ of patients (also including ypT 1$)^{8,19,20}$. The results of a number of similar trials are awaited (Greccar2, TREC). Currently, new trials are being conducted to randomize patients between standard treatment (i.e. TME without neoadjuvant therapy) and neoadjuvant (chemo) radiation followed by TEM or watchand-wait. The downside of this approach is that a sizeable portion of the patients who do not have a good response require immediate completion TME. These patients do not benefit from the neoadjuvant therapy but suffer from the added morbidity while they could have undergone TME without neoadjuvant therapy. A tool that predicts the response before the onset of treatment will better enable a differentiation between patients who will respond very well to CRT and those who will require TME. Also for patients with more locally advanced disease, response prediction could play a role in individualized treatment. For example, when a good response is predicted, intensification of treatment with a radiotherapy boost or chemotherapy could be considered to further increase the chance of organ-preservation ${ }^{21-24}$. On the other hand, if a poor response is predicted, systemic therapy and surgery are more rational treatment modalities than radiotherapy.

\section{Response prediction with imaging}

Other than the fact that smaller volumes are associated with a better response, it is currently not well understood why some patients show a dramatic response to treatment and others do not. Based on a morphological assessment of the primary tumor only, imaging cannot accurately predict the tumor response to therapy because most rectal adenocarcinoma, except for the mucinous tumors, have similar morphological MR features. MRI can however provide more than just morphological information, like in functional MRI techniques, that combine morphological features with quantifiable measurements of underlying tumor characteristics (biomarkers). Although DWI is a promising technique as a response measure after therapy, the role of diffusion in response prediction before the onset of treatment has yet to be determined. Some reports have shown promising results for diffusion ${ }^{25-27}$, but others could not confirm this difference between good and poor responding tumors measured before the onset of therapy ${ }^{17,28,29}$. A recent pooled-analysis of available literature showed an overall accuracy of 68\% for response prediction with DWI ${ }^{16}$. PET-imaging, which assesses tumor metabolic activity, is not accurate for response prediction before the start of 
treatment ${ }^{16}$, but is reported to be able to predict the tumor response at week 1-2 after the onset of treatment with overall accuracies of $81-95 \% 26,30,31$.

Tumor perfusion is an important component of tumor pathophysiology and could potentially contain information to predict the response to treatment. High perfusion in tumors is associated with a higher risk for distant metastasis and poorer survival outcomes ${ }^{32,33}$. However, the relationship between tumor perfusion and response to neoadjuvant therapy is not clear yet. Poor tumor perfusion results in hypoxic areas, which are known to be resistant to radiotherapy ${ }^{34}$. Also, hypoxia causes cells to stop or decrease cell proliferation ${ }^{35}$, possibly reducing the cytotoxic efficacy of chemotherapy, which is more effective against rapidly proliferating cells. This would indicate that poorly perfused tumors are associated with a poor response due to hypoxia. Tumors with a high perfusion are generally better oxygenated, which would imply a good response to radiotherapy. Furthermore, tumor perfusion is a prerequisite for the delivery and consequently the effectiveness of chemotherapy, thus a high perfusion could be associated with a good response to chemotherapy. On the other hand, high tumor perfusion indicates a high angiogenic activity, which is associated with a higher tumor aggressiveness.. This would mean that tumors with high perfusion are associated with a poor response. Keep in mind that vascularization and perfusion are two different definitions and a high vascularization does not automatically translate in high perfusion, since angiogenic vessels are leak and disorganized and often result in irregular blood flow and an heterogeneous blood distribution within the tissue.

Tumor perfusion as measured by dynamic contrast-enhanced MRI (DCE-MRI) has been investigated for the prediction of tumor response before the onset of therapy. The results of different studies are difficult to compare because different quantitative and semiquantitative analyses have been reported, and a high tumor perfusion has been reported to be associated with both poor ${ }^{36-38}$ and good ${ }^{39-44}$ tumor response. In chapter 6 we found that tumors characterized by a higher 'late slope', which implies a higher vascular permeability, were associated with poor response, hence supporting the hypothesis that tumors with high angiogenic activity are aggressive and poorly responding tumors. In chapter 7 we investigated the macro- and microvasculature in the mesorectum surrounding the tumor. A high microvasculature was associated with a poor tumor response, in accordance with our previous results on tumor perfusion. Interestingly, enhanced mesorectal macrovasculature was associated with a good tumor response, possibly referring to the importance of adequate delivery of chemotherapy.

Considering the current trend towards organ-preservation for early tumors, it would be interesting to investigate if DCE-MRI can also predict the response in early tumors and possibly select the patients who would benefit from neoadjuvant treatment. Hopefully, this diagnostic technique can be combined with the ongoing and future trials for neoadjuvant therapy in early tumors. 


\section{Future perspectives and recommendations}

Although organ-preservation in rectal cancer is still controversial, it is gaining interest from both clinicians and patients. Even though in this thesis we showed excellent long-term outcomes in a cohort study, data on more patients with a longer follow-up are required to provide more evidence on the oncological safety of this approach. Recently, a multicenter collaboration has started an international database aiming to concentrate data of the main organ-preservation initiatives throughout the world. Patient selection is crucial in organpreservation and many imaging techniques, both morphologic and functional, have been reported as valuable tools to assess tumor response after neoadjuvant chemoradiation. However, further large scale multicenter studies should be conducted to identify the most valuable imaging modalities that have the potential for implementation for nonexpert radiologists in daily practice. A recent study shows that the combination of clinical assessment and MRI (including DWI) results in a better accuracy to detect patients with a complete response compared to a single modality approach ${ }^{45}$. More combinations of several parameters, both imaging, clinical (e.g. digital rectal examination, endoscopy), and biomarkers should be investigated to result in a model with the highest accuracy of response assessment to enable optimal patient selection. Imaging parameters should be standardized before implementation on a large scale is possible, especially for quantitative parameters. Quantitative imaging assessment requires post-processing that is often time-consuming and can be technically challenging. Fully and semi-automated post-processing tools should be developed to facilitate implementation in routine practice.

In addition, imaging modalities for response prediction before the onset of therapy, and early during treatment, should be further explored. Prediction of the response before or early during treatment, could allow patient-tailored therapy with intensified, reduced, or alternative treatments. DCE-MRI seems promising for response prediction, and PET during therapy. With the development of PET-MRI, there is a new possibility to combine PET with DWI and DCE-MRI.

Based on all the above, organ-preservation for complete responders after neoadjuvant treatment appears to be a oncological safe and functionally good option as an alternative to the presently still standard indicated TME. Patients with a near complete response at initial response assessment could go for TEM or a second assessment 3 months later. These patients will however have a higher chance of having residual tumor, with a higher risk of a local regrowth during the first year of follow-up. Patient selection remains difficult and should be performed with caution, preferable by centers with expertise. 


\section{References}

1. Habr-Gama A, de Souza PM, Ribeiro U, Jr., et al. Low rectal cancer: impact of radiation and chemotherapy on surgical treatment. Dis Colon Rectum 1998; 41(9): 1087-96.

2. Habr-Gama A, Perez RO, Nadalin W, et al. Operative versus nonoperative treatment for stage 0 distal rectal cancer following chemoradiation therapy: long-term results. Annals of surgery 2004; 240(4): 711-7; discussion 7-8.

3. Habr-Gama A, Gama-Rodrigues J, Sao Juliao GP, et al. Local recurrence after complete clinical response and watch and wait in rectal cancer after neoadjuvant chemoradiation: impact of salvage therapy on local disease control. International journal of radiation oncology, biology, physics 2014; 88(4): 822-8.

4. Ayloor Seshadri R, Kondaveeti SS, Jayanand SB, et al. Complete clinical response to neoadjuvant chemoradiation in rectal cancers: can surgery be avoided? Hepatogastroenterology 2013; 60(123): 410-4.

5. Maas M, Nelemans PJ, Valentini V, et al. Long-term outcome in patients with a pathological complete response after chemoradiation for rectal cancer: a pooled analysis of individual patient data. The lancet oncology 2010; 11(9): 835-44.

6. Smith JD, Ruby JA, Goodman KA, et al. Nonoperative management of rectal cancer with complete clinical response after neoadjuvant therapy. Annals of surgery 2012; 256(6): 965-72.

7. Coco C, Rizzo G, Mattana C, et al. Transanal endoscopic microsurgery after neoadjuvant radiochemotherapy for locally advanced extraperitoneal rectal cancer: short-term morbidity and functional outcome. Surgical endoscopy 2013; 27(8): 2860-7.

8. Lezoche G, Baldarelli M, Guerrieri M, et al. A prospective randomized study with a 5-year minimum follow-up evaluation of transanal endoscopic microsurgery versus laparoscopic total mesorectal excision after neoadjuvant therapy. Surgical endoscopy 2008; 22(2): 352-8.

9. Perez RO, Habr-Gama A, Sao Juliao GP, Proscurshim I, Scanavini Neto A, Gama-Rodrigues J. Transanal endoscopic microsurgery for residual rectal cancer after neoadjuvant chemoradiation therapy is associated with significant immediate pain and hospital readmission rates. Dis Colon Rectum 2011; 54(5): 545-51.

10. Bujko K, Richter P, Smith FM, et al. Preoperative radiotherapy and local excision of rectal cancer with immediate radical re-operation for poor responders: a prospective multicentre study. Radiother Oncol 2013; 106(2): 198-205.

11. Perez RO, Habr-Gama A, Lynn PB, et al. Transanal endoscopic microsurgery for residual rectal cancer (ypT0-2) following neoadjuvant chemoradiation therapy: another word of caution. Dis Colon Rectum 2013; 56(1): 6-13.

12. Hupkens B, Martens M, Belgers E, et al. Quality of life of patients with rectal cancer treated by chemoradiation therapy alone and showing clinical complete response: a comparative study with patients treated by surgery. Colorectal Dis 2014; 16 (Suppl. 3): 29.

13. Vliegen RF, Beets GL, Lammering G, et al. Mesorectal fascia invasion after neoadjuvant chemotherapy and radiation therapy for locally advanced rectal cancer: accuracy of MR imaging for prediction. Radiology 2008; 246(2): 454-62.

14. Suppiah A, Hunter IA, Cowley J, et al. Magnetic resonance imaging accuracy in assessing tumour down-staging following chemoradiation in rectal cancer. Colorectal Dis 2009; 11(3): 249-53. 
15. Beets-Tan RG, Lambregts DM, Maas M, et al. Magnetic resonance imaging for the clinical management of rectal cancer patients: recommendations from the 2012 European Society of Gastrointestinal and Abdominal Radiology (ESGAR) consensus meeting. Eur Radiol 2013; 23(9): 2522-31.

16. Joye I, Deroose CM, Vandecaveye V, Haustermans K. The role of diffusion-weighted MRI and (18) F-FDG PET/CT in the prediction of pathologic complete response after radiochemotherapy for rectal cancer: a systematic review. Radiother Oncol 2014; 113(2): 158-65.

17. Curvo-Semedo L, Lambregts DM, Maas M, et al. Rectal cancer: assessment of complete response to preoperative combined radiation therapy with chemotherapy--conventional MR volumetry versus diffusion-weighted MR imaging. Radiology 2011; 260(3): 734-43.

18. Lambregts DM, Rao SX, Sassen S, et al. MRI and Diffusion-Weighted MRI Volumetry for Identification of Complete Tumor Responders After Preoperative Chemoradiotherapy in Patients With Rectal Cancer: A Bi-institutional Validation Study. Annals of surgery 2014; Epub ahead of print.

19. Garcia-Aguilar J, Shi Q, Thomas CR, Jr., et al. A phase II trial of neoadjuvant chemoradiation and local excision for T2N0 rectal cancer: preliminary results of the ACOSOG Z6041 trial. Ann Surg Oncol 2012; 19(2): 384-91.

20. Verseveld M, de Graaf EJ, Verhoef C, et al. Chemoradiation therapy for rectal cancer in the distal rectum followed by organ-sparing transanal endoscopic microsurgery (CARTS study). Br J Surg 2015; 102(7): 853-60.

21. Burbach JP, den Harder AM, Intven M, van Vulpen M, Verkooijen HM, Reerink O. Impact of radiotherapy boost on pathological complete response in patients with locally advanced rectal cancer: a systematic review and meta-analysis. Radiother Oncol 2014; 113(1): 1-9.

22. Habr-Gama A, Perez RO, Sabbaga J, Nadalin W, Sao Juliao GP, Gama-Rodrigues J. Increasing the rates of complete response to neoadjuvant chemoradiotherapy for distal rectal cancer: results of a prospective study using additional chemotherapy during the resting period. Dis Colon Rectum 2009; 52(12): 1927-34.

23. Gao YH, Lin JZ, An X, et al. Neoadjuvant sandwich treatment with oxaliplatin and capecitabine administered prior to, concurrently with, and following radiation therapy in locally advanced rectal cancer: a prospective phase 2 trial. International journal of radiation oncology, biology, physics 2014; 90(5): 1153-60.

24. Appelt AL, Ploen J, Harling H, et al. High-dose chemoradiotherapy and watchful waiting for distal rectal cancer: a prospective observational study. The lancet oncology 2015; 16(8): 919-27.

25. Intven M, Reerink O, Philippens ME. Diffusion-weighted MRI in locally advanced rectal cancer : pathological response prediction after neo-adjuvant radiochemotherapy. Strahlentherapie und Onkologie: Organ der Deutschen Rontgengesellschaft [et al] 2013; 189(2): 117-22.

26. Lambrecht M, Deroose C, Roels S, et al. The use of FDG-PET/CT and diffusion-weighted magnetic resonance imaging for response prediction before, during and after preoperative chemoradiotherapy for rectal cancer. Acta Oncol 2010; 49(7): 956-63.

27. Lambrecht M, Vandecaveye V, De Keyzer F, et al. Value of diffusion-weighted magnetic resonance imaging for prediction and early assessment of response to neoadjuvant radiochemotherapy in rectal cancer: preliminary results. International journal of radiation oncology, biology, physics 2012; 82(2): 863-70. 
28. Ha HI, Kim AY, Yu CS, Park SH, Ha HK. Locally advanced rectal cancer: diffusion-weighted MR tumour volumetry and the apparent diffusion coefficient for evaluating complete remission after preoperative chemoradiation therapy. Eur Radio/ 2013; 23(12): 3345-53.

29. Kim SH, Lee JY, Lee JM, Han JK, Choi BI. Apparent diffusion coefficient for evaluating tumour response to neoadjuvant chemoradiation therapy for locally advanced rectal cancer. Eur Radiol 2011;21(5): 987-95.

30. Goldberg N, Kundel Y, Purim O, et al. Early prediction of histopathological response of rectal tumors after one week of preoperative radiochemotherapy using 18 F-FDG PET-CT imaging. A prospective clinical study. Radiation oncology 2012; 7: 124.

31. Leccisotti L, Gambacorta MA, de Waure C, et al. The predictive value of (18)F-FDG PET/CT for assessing pathological response and survival in locally advanced rectal cancer after neoadjuvant radiochemotherapy. European journal of nuclear medicine and molecular imaging 2015; 42(5): 657-66.

32. Saclarides TJ, Speziale NJ, Drab E, Szeluga DJ, Rubin DB. Tumor angiogenesis and rectal carcinoma. Dis Colon Rectum 1994; 37(9): 921-6.

33. Sokmen S, Sarioglu S, Fuzun M, Terzi C, Kupelioglu A, Aslan B. Prognostic significance of angiogenesis in rectal cancer: a morphometric investigation. Anticancer research 2001; 21(6B): 4341-8.

34. Thomlinson RH, Gray LH. The histological structure of some human lung cancers and the possible implications for radiotherapy. Br J Cancer 1955; 9(4): 539-49.

35. Amellem O, Pettersen EO. Cell inactivation and cell cycle inhibition as induced by extreme hypoxia: the possible role of cell cycle arrest as a protection against hypoxia-induced lethal damage. Cell proliferation 1991; 24(2): 127-41.

36. DeVries AF, Kremser C, Hein PA, et al. Tumor microcirculation and diffusion predict therapy outcome for primary rectal carcinoma. International journal of radiation oncology, biology, physics 2003; 56(4): 958-65.

37. Kremser C, Trieb T, Rudisch A, Judmaier W, de Vries A. Dynamic T(1) mapping predicts outcome of chemoradiation therapy in primary rectal carcinoma: sequence implementation and data analysis. J Magn Reson Imaging 2007; 26(3): 662-71.

38. Sahani DV, Kalva SP, Hamberg LM, et al. Assessing tumor perfusion and treatment response in rectal cancer with multisection CT: initial observations. Radiology 2005; 234(3): 785-92.

39. George ML, Dzik-Jurasz AS, Padhani AR, et al. Non-invasive methods of assessing angiogenesis and their value in predicting response to treatment in colorectal cancer. Br J Surg 2001; 88(12): 1628-36.

40. Intven M, Reerink O, Philippens ME. Dynamic contrast enhanced MR imaging for rectal cancer response assessment after neo-adjuvant chemoradiation. J Magn Reson Imaging 2015; 41(6): 1646-53.

41. Lim JS, Kim D, Baek SE, et al. Perfusion MRI for the prediction of treatment response after preoperative chemoradiotherapy in locally advanced rectal cancer. Eur Radiol 2012; 22(8): 1693700.

42. Oberholzer K, Menig M, Pohlmann A, et al. Rectal cancer: assessment of response to neoadjuvant chemoradiation by dynamic contrast-enhanced MRI. J Magn Reson Imaging 2013; 38(1): 119-26. 
43. Tong T, Sun Y, Gollub MJ, et al. Dynamic contrast-enhanced MRI: Use in predicting pathological complete response to neoadjuvant chemoradiation in locally advanced rectal cancer. J Magn Reson Imaging 2015; 42(3): 673-80.

44. Zahra MA, Tan LT, Priest AN, et al. Semiquantitative and quantitative dynamic contrast-enhanced magnetic resonance imaging measurements predict radiation response in cervix cancer. International journal of radiation oncology, biology, physics 2009; 74(3): 766-73.

45. Maas M, Lambregts DM, Nelemans PJ, et al. Assessment of Clinical Complete Response After Chemoradiation for Rectal Cancer with Digital Rectal Examination, Endoscopy, and MRI: Selection for Organ-Saving Treatment. Ann Surg Oncol 2015; Epub ahead of print. 


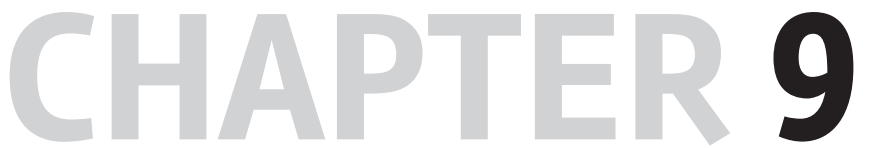

Valorisation 
Cancer is still one of the most leading causes of death is Europe and its incidence is expected to increase in the near future. Colorectal cancer is the second most common cause of cancerrelated death in both men and women. Of all colorectal cancers, approximately one third comprise rectal cancer, making rectal cancer an important health care problem. Symptoms that can occur in rectal cancer are bloody stools, change in size, shape, or frequency of stools, not being able to completely empty the bowel, abdominal pain or discomfort, unintended weight loss, and fatigue. Patients suspected for colorectal cancer will undergo an endoscopic examination of the colon combined with biopsies of any suspect tumoral lesions. When histopathological examination of these biopsies show cancer cells, the diagnosis of rectal cancer is confirmed. To evaluate the stage of disease, imaging is obtained of the local tumor but also of the liver and lungs, which are at risk for distant metastases. For assessment of the local extent of the tumor and possible invasion of local lymph nodes, MRI is performed.

Traditionally, rectal cancer was associated with a poor prognosis, mainly due to the high risk of a local recurrence of the tumor after surgery. A local recurrence is often diagnosed in an advanced stage when surgical resection is not possible anymore. It is associated with impaired quality of life, severe pain, and immobilization. Treatment often involves radiotherapy, chemotherapy, and/or extensive surgery (if operable). Fortunately, local recurrence rates have decrease significantly due to standardized surgical techniques and the introduction of pre-operative (chemo) radiotherapy. However, the benefit of preoperative treatment depends on the presence or absence of risk factors associated with a local recurrence. The national guidelines for rectal cancer treatment are based on these risk factors.

Patient with a low riskfor local recurrencewill undergo surgery without preoperativetreatment, patients with an intermediate risk are treated with a short course of preoperative radiation therapy followed by surgical resection, and patients with a high risk are treated with a long course of radiation therapy combined with chemotherapy followed by surgery. The main aim of the combined chemoradiotherapy is to induce tumor downsizing and downstaging to increase the chance of a complete resection of the tumor and lymph nodes and therefore reducing the risk of a local recurrence. In approximately $15-20 \%$ of the patients treated with preoperative chemoradiotherapy, no residual cancer cells are found at histopathological examination of the surgical specimen. This means that the cancer has disappeared completely as a result of the preoperative chemoradiotherapy. One could wonder whether major surgery is still required when the tumor has already disappeared, especially when considering the risks of surgery. An alternative strategy could be an organ-preserving treatment, such as a local excision of the remaining scar-tissue or even no surgery at all but a stringent follow-up. Benefits of this strategy are the omission of major surgery which is associated with morbidity, a temporary or permanent colostomy, functional impairment, and a mortality-risk of 4-12\%. The potential risk or organ-preservation is an undertreatment of wrongly selected patients who still have a viable tumor after chemoradiotherapy. Therefore, accurate patient selection is of the utmost importance. When patients with a persistent tumor after chemoradiotherapy 
are wrongly selected for organ-preservation, they are at risk of regrowth of the tumor and potentially at a greater risk for distant metastasis. Currently, organ-preserving treatment is still controversial in rectal cancer management and different strategies of patient selection and follow-up are being practiced.

\section{Relevance of scientific results in this thesis}

The results of this thesis show that organ-preservation is a feasible and oncologically safe option for selected patients. Organ-preservation has the potential to improve the quality of life of rectal cancer patients. Patients will not have to undergo surgical resection, and therefore they will not be exposed to the associated morbidity and mortality. Furthermore, the risk of a colostomy is prevented, resulting in an improved quality of life.

The results of this thesis are based on patients with locally advanced rectal cancer who were treated with neoadjuvant chemoradiotherapy as part of the national guidelines. However, this strategy could also work in earlier stages of rectal cancer, as was shown in several trials. Early rectal cancer will respond better to the chemoradiotherapy, therefore organpreservation could be become a feasible option for more patients (up to 66\% of the patients). Recently, the Netherlands introduced a national screening program for colorectal cancer. This will result in an increase in rectal cancer patients, especially in the rate of early rectal cancers. Both pre- and post-therapy prediction of response could play an important role in optimizing individualized treatment and improving quality of life.

In this thesis novel MRI techniques are described to improve patient selection for organpreserving treatment. When these sequences are further developed and optimized, MRI vendors can deliver these techniques on their MRI machines as a standard protocol.

In the current thesis several post-processing techniques have been explored, such as histogram analyses and subtraction images. Such tools, but also techniques like a (semi) automated contouring system, can be further developed and offered as part of postprocessing software. The continued development of both the MRI sequences and postprocessing software will be in close collaboration with the manufacturers and vendors.

\section{Target population}

According to the national guidelines, all patients with a high-risk tumor are treated with preoperative chemoradiotherapy followed by a surgical resection. Approximately $15-20 \%$ of these patients will have a complete response of the tumor and could be considered for an organ-preserving treatment. As mentioned before, organ-preservation could also be feasible for patients with an early stage of rectal cancer. 


\section{Innovation and future}

The principle of organ-preservation for selected rectal cancer patients has been shown to be feasible and oncologically safe, although data on more patients and longer follow-up is desirable. Recently, a multicenter collaboration has started an international database in which the data of main organ-preservation pioneers is centralized. Furthermore, data on functional outcome of organ-preservation is scarce. We have started a prospective study to monitor the quality of life and functional outcome of these patients at several time-points over a period of 5 years.

Accurate patient selection for organ-preservation remains crucial. Multicenter studies with both imaging and clinical response assessment are required to identify the most valuable modalities and in particular to identify the optimal combination of parameters.

Response prediction before the onset of treatment has been shown to be feasible in a selected patient group. Further validation in a larger cohort is necessary. Then the next step would be implementation in a clinical trial in which preoperative treatment is adapted according to the chance of a complete response, for example by intensification of preoperative treatment. 
Summary 


\section{Summary}

The aims of this thesis were to evaluate the oncological outcome of organ-preservation in rectal cancer management, to investigate how to improve patient selection by means of modern MRI techniques, and asses the feasibility of a novel MRI technique to predict the response before the onset of treatment.

\section{PART I: Organ preservation}

In chapter 2, the oncological results of our initially promising pilot results on watch-and-wait were investigated in a larger cohort. The second aim was to update the selection and inclusion criteria. Patient selection was performed with digital rectal examination, endoscopy, and state-of-the-art MRI. Patients with a clinical complete response were included in the watchand-wait regimen. Patients with a near clinical complete response were offered 3 options: standard surgical resection, TEM, or a second assessment 3 months later. One-hundred patients were included in an organ-preservation treatment, either watch-and-wait ( $n=85)$, or a TEM ( $n=15)$. The median follow-up was 38 months. Fifteen patients developed a local regrowth, all salvageable and all within 25 months. Five patients had distant metastases and four patients died. Three-year local regrowth free survival was $84 \%$ and overall survival was $96 \%$. Functional outcome was good since only $1 / 22$ patients in the watch-and-wait group suffered from major incontinence. Therefore, organ-preservation appears oncologically safe for selected rectal cancer patients.

\section{PART II: Response assessment after therapy}

Chapter 3 reviews the available literature on tumor size and volume measurements based on standard T2-weighted MRI for response assessment after chemoradiotherapy. Based on the results of this review we prospectively validated the cut-offs from the reviewed studies in a multicenter cohort and determined the optimal cut-offs to predict response. In total 14 papers reported three different measurement methods (length, 3D-size, and whole volume), and three different study outcomes (complete tumor response, tumor regression grade 1-2, and tumor-downstaging). Tumor length and 3D-size were not helpful to assess the tumor response. Whole volume measurements were promising, especially for assessing a complete tumor response with an overall accuracy of up to $80 \%$. However, since sensitivities remained low (14-21\%), its application in daily clinics remains limited.

Chapter 4 explores the potential of the novel MR technique "magnetization transfer" (MT) imaging to differentiate between residual tumor and fibrosis after neoadjuvant chemoradiotherapy. Regions-of-interest were measured on the MT images and correlated with the histopathology. This proof-of-principle showed a significantly higher MT ratio in regions of fibrosis (38\%) compared to regions of residual tumor (30\%, $p<0.001)$. The sensitivity and specificity were $88 \%$ and $90 \%$ for detecting fibrosis with MT. Therefore, MT ratio could be a promising tool to differentiate between fibrosis and residual tumor. 
In chapter 5, we evaluated the value of MT imaging to differentiate between patients with a good and a poor response after neoadjuvant therapy. The mean MT ratio showed an AUC of 0.65 and 0.87 for two readers. However, the $95^{\text {th }}$ percentile of the MT ratio rendered an AUC of 0.75-0.88 with a good interobserver agreement (ICC 0.80). Magnetization transfer imaging seems a promising tool, but it should be further explored in a larger patient cohort.

\section{PART III: Response prediction before therapy}

Chapter 6 focuses on the performance of dynamic contrast enhanced (DCE) MRI to predict tumor response before the onset of neoadjuvant treatment. Thirty patients underwent DCEMRI before and after neoadjuvant chemoradiotherapy. Several kinetic parameters were derived from the time-enhancement curves both before and after therapy, and their relative difference between pre- and post-therapy was calculated. Only the parameter 'late slope' measured before the onset of therapy could predict a good response (tumor regression grade 1 -2) with a sensitivity of $92 \%$ and specificity of $82 \%$.

In Chapter 7 the macroscopic and microscopic parameters of the mesorectal vasculature before therapy and its relation to tumor response is investigated. This showed that the macroscopic vasculature was enhanced in the mesorectum adjacent to the tumor compared to mesorectal vasculature surrounding the normal rectal wall. Macrovasculature was also enhanced in node-positive disease compared to node-negative patients. Furthermore, good responders showed an enhanced macroscopic vasculature compared to poor responders. Thus, DCE-MRI shows a potential for response prediction, but further validation in larger patient cohort is warranted to confirm the diagnostic value. 
Samenvatting 


\section{Samenvatting}

De doelstellingen van deze thesis waren het evalueren van de oncologische uitkomst van orgaan-preservering als behandeling van het rectumcarcinoom, onderzoeken op welke manier de patiënten selectie verbeterd kan worden met behulp van moderne MRI technieken en het evalueren van de potentie van een nieuwe MRI techniek ter voorspelling van de respons voorafgaand aan de start van de therapie.

\section{DEEL I: Orgaan-preservatie}

In hoofdstuk 2 werden de oncologische resultaten van onze eerste veelbelovende studie onderzocht in een groter cohort. Het tweede doel was het updaten van de selectie en inclusie criteria. De selectie van patiënten werd verricht door middel van een rectaal toucher, endoscopie en state-of-the-art MRI. Patiënten met een klinisch complete respons werden geïncludeerd in het 'watch-and-wait' stramien. Patiënten met een bijna klinisch complete respons werden 3 opties aangeboden: standaard operatie, TEM of een tweede beoordeling na 3 maanden. Honderd patiënten werden geïncludeerd in een orgaan-preserverende behandeling; watch-and-wait ( $n=85)$ of een TEM $(n=15)$. De mediane duur van opvolgen was 38 maanden. Vijftien patiënten kregen een lokale teruggroei (allen binnen 25 maanden), welke in alle gevallen nog geopereerd konden worden. Vijf patiënten hadden metastasen op afstand en vier patiënten zijn overleden. Drie-jaars lokale teruggroei-vrije overleving was $84 \%$ en de totale overleving was $96 \%$. Slechts één van de 22 ondervraagde patiënten uit de watch-and-wait groep, had last van ernstige incontinentie. Derhalve is orgaan-preservatie een oncologisch veilige optie voor geselecteerde patiënten met een rectumcarcinoom.

\section{DEEL II: Respons evaluatie na therapie}

Hoofdstuk 3 geeft een overzicht van de beschikbare literatuur over tumor grootte en volume metingen gebaseerd op standaard T2-gewogen MRI voor respons evaluatie na chemoradiotherapie. Uit dit overzicht werden grootte- en volume-afkapwaardes afgeleid, welke vervolgens prospectief werden gevalideerd in een multicenter cohort waarmee de optimale afkapwaarden werden geïdentificeerd. In totaal waren er 14 artikelen die 3 verschillende meetmethodes (lengte, 3D-grootte en totaal volume) en 3 verschillende uitkomstmaten gebruikten (complete tumor respons, tumor regressie graad 1-2 en tumordownstaging). Tumor lengte en 3D-grootte bleken niet behulpzaam voor tumor respons evaluatie. Totale volume metingen waren veelbelovend, met name voor het evalueren van een complete tumor respons met een accuratesse tot $80 \%$. Echter, aangezien de sensitiviteit laag bleef (14-21\%) lijkt de applicatie in de dagelijkse praktijk beperkt.

Hoofdstuk 4 onderzoekt het potentieel van de nieuwe MRI techniek "magnetization transfer" (MT) imaging voor het differentiëren van resttumor en fibrose na neoadjuvante chemoradiotherapie. 'Regions-of-interest' werden gemeten op de MT beelden en gecorreleerd 
met de histopathologie. Dit toonde een significant hogere MT ratio in regio's met fibrose (38\%) vergeleken met resttumor (30\%, p <0.001). De sensitiviteit en specificiteit waren $88 \%$ en $90 \%$ voor het detecteren van fibrose met MT. Derhalve zou MT imaging veelbelovend kunnen zijn om te differentiëren tussen fibrose en resttumor.

In hoofdstuk 5 hebben we de waarde van MT imaging geëvalueerd om te differentiëren tussen patiënten met een goede en een slechte respons na neoadjuvante therapie. De gemiddelde MT ratio toonde een AUC van 0.65 en 0.87 voor twee observanten. Echter, de $95^{\mathrm{e}}$ percentiel van de MT ratio toonde een AUC van 0.75-0.88 met een goede interobservant overeenkomst (ICC 0.80). Magnetization transfer imaging lijkt een veelbelovende modaliteit, maar verder onderzoek in een grotere patiëntengroep is nodig.

\section{DEEL III: respons predictie vooraf aan therapie}

Hoofdstuk 6 richt zich op 'dynamic contrast enhanced' (DCE) MRI voor het voorspellen van de tumor respons voorafgaand aan de start van neoadjuvante therapie. Dertig patiënten kregen DCE-MRI voorafgaand en na de neoadjuvante chemoradiotherapie. Een aantal kinetische parameters werden afgeleid van de 'time-enhancement' curves zowel vooraf als na de therapie en het relatieve verschil tussen pre- en post-therapie werd berekend. Enkel de parameter 'late slope' gemeten vooraf aan de therapie, kon een goede respons (TRG 1-2) voorspellen met een sensitiviteit van $92 \%$ en specificiteit van $82 \%$.

In hoofdstuk 7 werden de macroscopische en microscopische parameters van de mesorectale vasculatuur vooraf aan therapie onderzocht, en hun relatie tot de tumor respons. Dit toonde dat the macroscopische vasculatuur was toegenomen in het mesorectum rondom de tumor vergeleken met het mesorectum rondom de normale rectum wand. Macrovasculatuur was ook toegenomen in klier-positieve ziekte vergeleken met klier-negatieve patiënten. Daarbij hadden goede responders toegenomen macrovasculatuur vergeleken met slechte responders. Dus, DCE-MRI heeft potentieel voor het respons predictie, maar verdere validatie in een grotere patiëntengroep is nodig om de diagnostische waarde vast te stellen. 
Dankwoord 


\section{Dankwoord}

En dan natuurlijk nog het gedeelte dat het meeste gelezen wordt. Zo niet, het enige deel dat gelezen wordt: het dankwoord. En uiteraard ga ik ook mensen vergeten te noemen, anders zou het geen echt dankwoord zijn. Maar sorry daarvoor alvast.

Allereerst wil ik graag alle patiënten bedanken die aan dit onderzoek hebben meegewerkt. Zonder jullie bereidwilligheid had deze thesis nooit tot stand kunnen komen.

Prof. Dr. Regina Beets-Tan, beste Regina, jouw enthousiasme en passie voor dit onderzoek is onuitputtelijk. Ik was meteen overtuigd dat ik op de goede plek was beland en ik werd al snel opgenomen in het 'recteam'. Ik heb zeer veel respect voor je hoe je alles weet te managen ondanks je drukke schema, maar toch altijd ergens de tijd vindt om snel en kritisch naar papers te kijken. Je wist me niet te overtuigen om radioloog te worden (ondanks je vele pogingen), maar ik heb wel enorm veel van je geleerd. Bedankt voor de kansen die me hebt gegeven en je vertrouwen. Je bent zelf net aan een nieuwe uitdaging begonnen in Amsterdam, heel veel succes!

Prof. Dr. Geerard Beets, beste Geerard, ik vind het een eer dat ik deel heb mogen uitmaken van het 'wait-and-see' project, recent omgedoopt tot 'watch-and-wait'. Heel bijzonder hoe jij je als chirurg hard maakt om patiënten niet te opereren. Ook bij jou kon ik altijd aankloppen, wel met het risico dat ik met nog meer nieuwe (soms wilde) ideeën en projecten weer vertrok. Bedankt voor je begeleiding en veel succes in Amsterdam.

Dr. Doenja Lambregts, beste Doenja, toen je zelf nog officieel moest promoveren, bleek al snel dat je een uitstekende begeleider was. Altijd snel en kritisch naar papers kijken, maar ook gewoon een gezellige collega. Gelukkig kon ik jou ook af en toe helpen, door bomen van drieën te onderscheiden bijvoorbeeld. Heel erg bedankt voor je goede en prettige begeleiding. Ook jij veel succes in Amsterdam!

Prof. Dr. Jos van Engelshoven, het is een eer dat $u$ voorzitterschap van mijn beoordelingscommissie op zich heeft willen nemen. Ik wil $u$ en de andere leden van de beoordelingscommissie, Prof. Dr. Nicole Bouvy, Prof. Dr. Walter Backes, Prof. Dr. Jaap Stoker en Prof. Dr. Marcel Verheij, alsmede de leden van de oppositiecommissie, hartelijk bedanken voor de kritische beoordeling van mijn proefschrift.

Dr. Luc Heijnen, beste Luc, mijn 'work-hubbie', paranimf of 'partner-in-crime' zoals jij me noemde. Inmiddels al anderhalf jaar geleden dat we samen hebben gewerkt, maar ik denk nog altijd met plezier terug aan die periode. Helaas niet tegelijk gepromoveerd, maar het scheelt maar één dag! (ok, en een jaar...) Je was nooit te beroerd om eens bij te springen en 
ik kon altijd op je rekenen. Bedankt voor de fijne samenwerking en die ontelbare keren dat je me aan een naam bij een gezicht kon helpen. Veel succes in Amsterdam en met de opleiding!

De overige leden van het 'Recteam': Max, Sanne en Elleke als eerste generatie. Monique als mijn voorganger, bedankt voor je begeleiding en statistiek-kunsten natuurlijk. Miriam en Britt, van semi-arts naar mijn opvolgers. Rianne, het was even wennen na een jaartje kliniek, maar gelukkig heb je je draai gevonden. Joost en Marit, de allernieuwste aanwinst van het recteam, veel succes in Amsterdam. Het is fantastisch om deel uit te maken van het recteam! Niet alleen op de werkvloer, maar ook op de dansvloer (of karaokevloer...)!

Ik wil graag de afdeling radiologie bedanken voor de leuke sfeer. Frans en Max, ontzettend bedankt voor het alsmaar tellen van lymfeklieren en scoren van MRI's. Ik wil ook de abdomenradiologen bedanken voor hun inzet: Janneke, Miguel, Stanley, Ulrich en Vincent.

Prof. Dr. Joachim Wildberger, het afdelingshoofd van de radiologie, altijd geïnteresseerd in de vorderingen. Bedankt.

De dames van het secretariaat radiologie wil natuurlijk bedanken voor hun support, maar vooral voor de gezellige praatjes tussendoor. Elfie, Monique, Christianne, Peggy, Lut en Margriet, bedankt!

Ik wil het personeel van de radiologie balie en planning bedanken, met in het bijzonder Sylvia, Guus, Jan, Irene, Daisy, Annie en Bea voor jullie bodemloze geduld als we weer eens met een stapel aanvragen kwamen aanzetten.

Marc en Jos, bij jullie kon ik met al mijn computer problemen terecht. Bedankt! Roland (en eerst nog eventjes Henk), als teamleidervan team 5 heb je heel wat geduld moeten hebben met ons. Bedankt voor al je hulp om het elke keer toch voor elkaar te krijgen. Ik wil alle laboranten van team 5 bedanken voor jullie hulp en inzet om de patiënten te scannen met de alsmaar veranderende protocollen.

De afdeling chirurgie, met in het bijzonder de GE-chirurgen (Laurents Stassen, Marjolein Smidt, Kristien Keymeulen, Stephanie Breukink, Jarno Melenhorst), bedankt voor jullie inzet om de patiënten te begeleiden in het wait-and-see traject. Marjolein wil ik tevens bedanken voor je enthousiasme en passie voor je vak en onderzoek waarmee je mij hebt aangestoken toen ik als student voor je deur stond.

Ook wil ik de afdelingen pathologie, MDL, oncologie, radiotherapie (Maastro) en GROW bedanken. En de dames en heer van DataCenter Maastro voor al jullie inzet en fijne samenwerking.

Christel Gielen, waar zouden we zijn zonder jouw inzet en begeleiding van de patiënten. Bedankt voor de plezierige samenwerking.

A special thanks to Nikos in Greece for all your support and collaboration. And I would like to thank Ewelina for your expertise and guidance. 
Alle radiologen, chirurgen, MDL-artsen, verpleegkundigen die aan de MRI2-studie en de Waitand-See studie hebben meegewerkt, bedankt!

Ik wil graag Vincent Vandecaveye en Joris van der Broek bedanken voor de prettige samenwerking.

Alle collega-onderzoekers van zowel de radiologie als de chirurgie, ontzettend bedankt voor de gezellige tijd de afgelopen jaren.

Alle assistenten en chirurgen in het Zuyderland te Sittard, bedankt voor jullie geduld tijdens mijn eerste paar maanden weer terug in de kliniek.

MAFS, bedankt dat jullie toch braaf naar mijn 'saaie onderzoeksverhalen' wilden luisteren terwijl jullie al gave en spannende verhalen uit de kliniek hadden. Maar gelukkig kan ik nu eindelijk meepraten! Annemiek, bedankt dat jij mijn paranimf wil zijn. Bijna hetzelfde als ceremoniemeester toch?

Natuurlijk wil ik mijn ouders, broers en schoonzus bedanken voor al hun steun de afgelopen jaren. Het heeft onder andere geleid tot bijzondere Sinterklaas gedichten met Gadofosveset erin. Ook wil ik mijn schoon(stief)ouders bedanken voor jullie support.

Lieve Elwin, bedankt voor je onvoorwaardelijke steun en vertrouwen in me. Aan een half woord heb je al genoeg, je staat altijd voor me klaar. Hopelijk kan ik de komende periode hetzelfde voor jou betekenen als jij naar je boekje toe gaat werken. 


\section{Curriculum Vitae}




\section{Curriculum Vitae}

Milou H. Martens was born on July 13th 1987, in Heemstede, the Netherlands. In 2005 she graduated from the Stedelijk Gymnasium Haarlem, and in the same year she moved to Maastricht to start her medical study at the Faculty of Medicine at Maastricht University. During her medical training, she started with her first research project in the surgery department of the Maastricht University Medical Center under supervision of dr. Marjolein Smidt. During this period, she made her first encounter with the research team of Prof. Dr. Regina Beets-Tan and Prof. Dr. Geerard Beets.

In 2011, immediately after obtaining her medical doctor

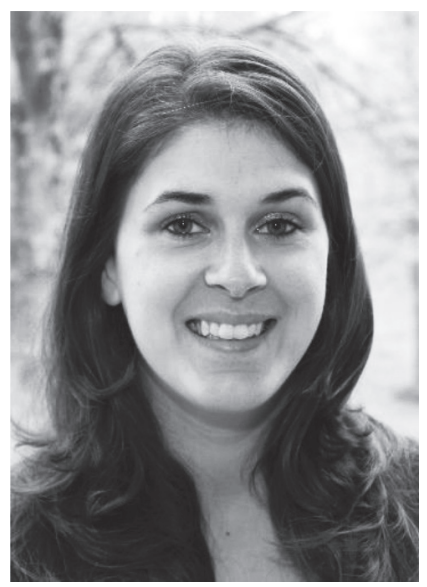
degree, she started working as a PhD student at the GROW School for Oncology and Developmental Biology under supervision of Prof. Dr. Regina BeetsTan, Prof. Dr. Geerard Beets, and Dr. Doenja Lambregts. During this period she was nominated as an "ESGAR top 20 presenter" (2012 and 2014), was granted the "Rising Star travel grant ECR 2014", and was granted the "Six Best Papers Session, ESCP 2015".

In June 2015, she started working as a surgical resident at the Zuyderland Medical Center in Sittard. 


\section{List of publications}




\section{List of publications}

\section{This thesis}

MH Martens, MM van Heeswijk, JJ van den Broek, S-X Rao, V Vandecaveye, RA Vliegen, WH Schreurs, GL Beets, DMJ Lambregts, RGH Beets-Tan. Prospective multicenter validation study of MRI volumetry for response assessment after preoperative chemoradiation in rectal cancer: can the results in literature be reproduced? International Journal of Radiation Oncology, Biology, Physics, 2015 Sept 15. In press accepted manuscript

E Kluza, MH Martens, J-PJE Kleijnen, D Rennspiess, M Maas, CRLPN Jeukens, RG Riedl, A zur Hausen, GL Beets, RGH Beets-Tan. Non-invasive MR assessment of macroscopic and microscopic vascular abnormalities in the rectal tumor-surrounding mesorectum. European Radiology 2015 Aug 30. [Epub ahead of print]

MH Martens, DM Lambregts, N Papanikolaou, S Alefantinou, M Maas, GC Manikis, R Riedl, GL Beets, RGH Beets-Tan. Magnetization transfer imaging to assess tumour response after chemoradiotherapy in rectal cancer. European Radiology. 2015 Jun 12 [Epub ahead of print]

MH Martens, S Subhani, LA Heijnen, DMJ Lambregts, J Buijsen, M Maas, RG Riedl, CR Jeukens, GL Beets, E Kluza, RGH Beets-Tan. Can perfusion MRI predict response to preoperative treatment in rectal cancer? Radiotherapy \& Oncology; 2015 Feb;114(2):218-23

MH Martens, DMJ Lambregts, N Papanikolaou, LA Heijnen, RG Riedl, A zur Hausen, M Maas, GL Beets, RGH Beets-Tan. Magnetization Transfer Ratio: A potential biomarker for the assessment of postradiation fibrosis in patients with rectal cancer. Investigative Radiology; January2014;49(1):29-34

MH Martens, M Maas, LA Heijnen, DMJ Lambregts, JWA Leijtens, LPS Stassen, SO Breukink, C Hoff, HJ Belgers, J Melenhorst, RLH Jansen, J Buijsen, AGM Hoofwijk, RGH Beets-Tan, GL Beets. Organ preservation in 100 clinical complete responders after neoadjuvant treatment for rectal cancer: long-term oncological and functional outcome.

Submitted 


\section{Other}

M Maas, DMJ Lambregts, PJ Nelemans, LA Heijnen, MH Martens, JWA Leijtens, M Sosef, KW Hulsewé, C Hoff, SO Breukink, LPS Stassen, RGH Beets-Tan, GL Beets. Assessment of Clinical Complete Response After Chemoradiation for Rectal Cancer with Digital Rectal Examination, Endoscopy, and MRI: Selection for Organ-Saving Treatment._Ann Surg Oncol. 2015 Jul 22. [Epub ahead of print]

DMJ Lambregts, MH Martens, RC Quah, K Nikiforaki, LA Heijnen, CH Dejong, GL Beets, K Marias, N Papanikolaou, RGH Beets-Tan. Whole-liver diffusion-weighted MRI histogram analysis: effect of the presence of colorectal hepatic metastases on the remaining liver parenchyma. Eur J Gastroenterol Hepatol. 2015 Apr;27(4):399-404

MH Martens, DMJ Lambregts, E Kluza, RGH Beets-Tan. Tumor response to treatment: Prediction and assessment. Current Radiology Reports. 2014

S-X Rao, DMJ Lambregts, RS Schnerr, W van Ommen, TJA van Nijnatten, MH Martens, LA Heijnen, WH Backes, C Verhoef, M-S Zeng, GL Beets, RGH Beets-Tan. Whole-liver CT texture analysis in colorectal cancer: Does the presence of liver metastases affect the texture of the remaining liver? United European Gastroenterol J. 2014 Dec;2(6):530-8

DMJ Lambregts, S-X Rao, S Sassen, MH Martens, LA Heijnen, J Buijsen, M Sosef, GL Beets, RA Vliegen, RGH Beets-Tan. MRI and Diffusion-Weighted MRI Volumetry for Identification of Complete Tumor Responders After Preoperative Chemoradiotherapy in Patients with Rectal Cancer- a Bi-institutional Validation Study. Annals of Surgery; Sep 2014 [Epub ahead of print]

LA Heijnen, M Maas, MJ Lahaye, U Lalji, DM Lambregts, MH Martens, RG Riedl, GL Beets, RGH Beets-Tan. Value of gadofosveset-enhanced MRI and multiplanar reformatting for selecting good responders after chemoradiation for rectal cancer. European Radiology; 2014 Aug;24(8):1845-52

LA Heijnen, DMJ Lambregts, MH Martens, M Maas, FC Bakers, VC Cappendijk, P Oliveira, G Lammering, RG Riedl, GL Beets, RGH Beets-Tan. Performance of gadofosveset-enhanced MRI for staging rectal cancer nodes: can the initial promising results be reproduced? European Radiology; 2014 Feb;24(2):371-9

DR van Mierlo, TR Lopez Penha, RJ Schipper, MH Martens, J Serroyen, MB Lobbes, EM Heuts, $S$ Tuinder, ML Smidt. No increase of local recurrence rate in breast cancer patients treated with skin-sparing mastectomy followed by immediate breast reconstruction. The Breast. 2013 Dec;22(6):1166-70 
LA Heijnen, DMJ Lambregts, D Mondal, MH Martens, RG Riedl, GL Beets, RGH Beets-Tan. Diffusion-weighted MR imaging in primary rectal cancer staging demonstrates but does not characterise lymph nodes. European Radiology; 2013 Dec;23(12):3354-60.

DMJ Lambregts, LA Heijnen, M Maas, IJG Rutten, MH Martens, WH Backes, RG Riedl, FCH Bakers, VC Cappendijk, GL Beets, RGH Beets-Tan. Gadofosveset-enhanced MRI for the assessment of rectal cancer lymph nodes: predictive criteria. Abdominal Imaging. 2013 Aug;38(4):720-7

E Kluza, ED Rozeboom, M Maas, MH Martens, DMJ Lambregts, J Slenter, GL Beets, RGH BeetsTan. T2 weighted signal intensity evolution may predict pathological complete response after treatment for rectal cancer. European Radiology; Jan 2013;23(1):253-61

MH Martens, LM van Roozendaal, LJ Schouten, JJR Hermans, A Bast, MP Weijenberg, ML Smidt. Gebruik van complementaire en alternatieve medicijnen door patiënten met mammacarcinoom. Nederlands tijdschrift voor Oncologie; Maart 2013;10(2):66-73

DMJ Lambregts, MJ Lahaye, LA Heijnen, MH Martens, M Maas, GL Beets, RGH Beets-Tan. Diffusion-weighted MRI for the detection of local recurrences after standard surgical treatment and after organ saving treatment for rectal cancer

Submitted

LA Heijnen, DMJ Lambregts, MJ Lahaye, T van Nijnatten, MH Martens, S-X Rao, RG Riedl, J Buijsen, GL Beets, RGH Beets-Tan. Good and complete responding locally advanced rectal tumors after chemoradiotherapy: where are the residual positive nodes located?

Submitted 